\title{
Dirac Optimal Reduction
}

\section{M. Jotz ${ }^{1}$ and T. S. Ratiu ${ }^{2}$}

${ }^{1}$ Mathematisches Institut, Georg August Universität Göttingen, Bunsenstrasse 3-5, 37073 Göttingen, Germany and ${ }^{2}$ Section de Mathématiques and Bernouilli Center, Ecole Polytechnique Fédérale de Lausanne, 1015 Lausanne, Switzerland

Correspondence to be sent to: mjotz@uni-math.gwdg.de

The purpose of this paper is to generalize the (Poisson) Optimal Reduction Theorem in Ortega and Ratiu [Momentum Maps and Hamiltonian Reduction. Progress in Mathematics 222. Boston, MA: Birkhäuser. xxxiv, 497pp., 2004] to general proper Lie group actions on Dirac manifolds, formulated both in terms of point and orbit reduction. A comparison to general standard singular Dirac reduction is given emphasizing the desingularization role played by optimal reduction.

\section{Introduction}

Dirac structures, introduced in [9] and systematically investigated for the first time in [8], have a wide range of applications in geometry and theoretical physics. They include 2-forms, Poisson structures, foliations and also provide a convenient geometric setting for the theory of nonholonomic systems and circuit theory. The study of sub-objects and quotient objects in the Dirac category, central in the theory of reduction, is of particular interest.

Received July 18, 2011; Revised October 31, 2011; Accepted November 18, 2011

Communicated by Prof. Anton Alekseev

(C) The Author(s) 2012. Published by Oxford University Press. All rights reserved. For permissions, please e-mail: journals.permissions@oup.com. 
Recent years have seen a significant development of Dirac structures from the geometric point of view as well as in applications. In the presence of symmetry, one can perform reduction to eliminate variables; see [2, 4, 6, 8, 16, 19, 31, 32], for the regular case and $[3,17,18]$, for the singular situation. All these reduction procedures are in the spirit of Poisson reduction [12, 15, 22, 25].

In symplectic reduction, a central role is played by the momentum map. While its existence in the symplectic category is not guaranteed, in concrete applications, it is rarely the case that a symplectic Lie group action fails to admit a momentum map. The situation is, however, drastically different in the Poisson category, where the existence of the momentum map imposes unreasonable constraints on the symmetries. Because of that, Ortega and Ratiu [23] introduced the optimal momentum map, a conservation law of a Poisson symmetry, that is always defined and has values in a topological space. A reduction method based on the optimal momentum map was proved.

In this paper, we generalize the optimal momentum map and the optimal reduction procedure in [23] to closed Dirac manifolds . (Historically, "closed" and "integrable" Dirac manifolds were synonyms. However, due to the relation of Dirac geometry to groupoids, an "integrable" Dirac structure is one that, viewed as an algebroid, integrates to a groupoid. This is the reason why, in this paper, we use exclusively the term "closed"). As we shall see, with necessary assumptions and appropriately extended definitions, this important desingularization method works also for Dirac manifolds. The power of optimal reduction can be immediately seen, already for free actions (3), by noting that it has as a trivial corollary, the stratification in presymplectic leaves of a closed Dirac manifold.

Singular optimal reduction is carried out in two steps. First, one considers appropriately chosen distributions jointly defined by the symmetry group and the Dirac structure. In the free case, this reduces to one distribution that is automatically integrable in the sense of Stefan and Sussmann if the hypotheses for regular/standard Dirac reduction are satisfied. In the nonfree case, this is no longer true, in general. However, if these generalized distributions are integrable, their leaves define the level sets of corresponding natural optimal momentum maps.

Second, one passes to the quotient and constructs on it the reduced Dirac structure. It is not possible to extend this result in a naive manner to nonclosed Dirac structures because the first consequence of nonclosedness is the nonintegrability of the projection of the Dirac structure on its tangent part, and hence, in general, the distribution used in the previously described reduction process is also nonintegrable. 


\subsection{Outline of the paper}

In Section 2.1, we briefly review the relevant definitions and properties of generalized distributions; special attention is given to integrability conditions for tangent distributions. Section 2.2 recalls the general theory of Dirac structures and Dirac actions of Lie groups and Lie algebras. The necessary material from the theory of proper Lie group actions is reviewed in Section 2.3 and the concept of descending sections of the Pontryagin bundle is recalled.

In Section 3, we summarize the results obtained later on in the particular case of a free Lie group action. No proofs are provided since they will be done for the general case in subsequent sections. Nevertheless, we believe that this section is helpful, since it illustrates the much more complicated general theory in a simple case; the distribution appearing here is the image of an appropriately chosen Lie algebroid by its anchor map.

Section 4 is devoted to the study of two special distributions that are crucial in the reduction procedure; they are called optimal distributions and are associated to orbit and isotropy types, respectively. Under the necessary conditions for standard singular reduction, these two optimal distributions are algebraically involutive and a hypothesis is given so that the one associated to isotropy types is integrable.

In Section 5, we introduce and study two optimal momentum maps, objects always available for canonical Lie group actions on closed Dirac manifolds if the optimal distributions are spanned by their descending sections and are integrable. If the Dirac structure comes from a Poisson manifold, these conditions are always satisfied and the two optimal momentum maps coincide. Optimal reduction is presented in Section 6. Two point optimal reduction theorems associated to the two optimal momentum maps are proved. In addition, an optimal orbit reduction theorem is presented and is shown that the three reduction procedures, that is, the two optimal point reduction and the optimal orbit reduction, are equivalent.

In Section 7, we show that standard and optimal Dirac reductions are equivalent under appropriate hypotheses. Section 8 is devoted to the study of several examples illustrating the theory.

A first appendix recalls an averaging method for vector fields, 1-forms and functions on a manifold acted upon properly by a Lie group. A second appendix summarizes

the technical results used to compute the symmetry invariant generators for the set of invariant vector fields used in the examples. 


\subsection{Notation and conventions}

We write $C^{\infty}(M)$ for the sheaf of local functions on $M$. That is, an element $f \in C^{\infty}(M)$ is a smooth function $f: U \rightarrow \mathbb{R}$, with $U$ an open subset of $M$. In the same manner, if $E$ is a vector bundle over $M$, or a generalized distribution on $M$, we denote by $\Gamma(E)$ the set of local sections of $E$. In particular, the sets of local vector fields and 1-forms on $M$ will be denoted by $\mathfrak{X}(M)$ and $\Omega^{1}(M)$, respectively. We will write $\operatorname{Dom}(\sigma)$ for the open domain of definition of $\sigma \in \Gamma(E)$.

The Lie group $G$ is always assumed to be connected; $\mathfrak{g}$ denotes its Lie algebra. All $G$-actions on $M$ are smooth and are denoted by $\Phi: G \times M \rightarrow M,(g, m) \mapsto g m=g \cdot m=$ $\Phi_{g}(m)$, for all $g \in G$ and $m \in M$. If $\xi \in \mathfrak{g}$, then $\xi_{M} \in \mathfrak{X}(M)$ defined by $\xi_{M}(m):=\left.\frac{\mathrm{d}}{\mathrm{d} t}\right|_{t=0} \exp (t \xi)$. $m$ is called the infinitesimal generator or fundamental vector field defined by $\xi$.

A section $X$ of $T M$ (respectively, $\alpha$ of $T^{*} M$ ) is called $G$-invariant, if $\Phi_{g}^{*} X=X$

(respectively, $\Phi_{g}^{*} \alpha=\alpha$ ) for all $g \in G$. Here, the vector field $\Phi_{g}^{*} X$ is defined by $\Phi_{g}^{*} X=T \Phi_{g^{-1}} \circ$ $X \circ \Phi_{g}$, that is, $\left(\Phi_{g}^{*} X\right)(m)=T_{g m} \Phi_{g^{-1}} X(g m)$, for all $m \in M$.

Recall that a subset $N \subset M$ is an initial submanifold of $M$ if $N$ carries a manifold structure such that the inclusion $\iota: N \hookrightarrow M$ is a smooth immersion and satisfies the following condition: for any smooth manifold $P$, an arbitrary map $g: P \rightarrow N$ is smooth if and only if $\iota \circ g: P \rightarrow M$ is smooth; in this case, $\iota$ is said to be a regular immersion. The notion of initial submanifold lies strictly between those of injectively immersed and embedded submanifolds.

\section{Generalized Distributions, Symmetries, and Standard Dirac Reduction}

\subsection{Generalized distributions}

The Pontryagin bundle $\mathrm{P}_{M}$ of a smooth manifold $M$ is the direct sum $\mathrm{P}_{M}=T M \oplus T^{*} M$. A generalized distribution $\Delta$ on $M$ is a subset $\Delta$ of $\mathrm{P}_{M}$ such that for each $m \in M$, the set $\Delta(m):=\Delta \cap \mathrm{P}_{M}(m)$ is a vector subspace of $\mathrm{P}_{M}(m)=T_{m} M \times T_{m}^{*} M$. The rank of $\Delta$ at $m \in M$ is $\operatorname{dim} \Delta(m)$. A point $m \in M$ is a regular point of the distribution $\Delta$ if there exists a neighborhood $U$ of $m$ such that the rank of $\Delta$ is constant on $U$. Otherwise, $m$ is a singular point of the distribution.

A local differentiable section of $\Delta$ is a smooth section $\sigma \in \Gamma\left(\mathrm{P}_{M}\right)$ defined on some open subset $U \subset M$ such that $\sigma(u) \in \Delta(u)$, for each $u \in U$; the open domain of definition of $\sigma$ is denoted by $\operatorname{Dom}(\sigma)$. Let $\Gamma(\Delta)$ be the space of differentiable local sections of $\Delta$. A generalized distribution is said to be differentiable or smooth if, for every point $m \in M$ and every vector $v \in \Delta(m)$, there is a differentiable section $\sigma \in \Gamma(\Delta)$ defined on an open 
neighborhood $U$ of $m$ such that $\sigma(m)=v$. A subset $S \subseteq \Gamma\left(\mathrm{P}_{M}\right)$ is said to span the smooth generalized distribution $\Delta$ if $\Gamma(\Delta)=\operatorname{span}_{C^{\infty}(M)}(S) ; S$ spans pointwise $\Delta$ if for all $m \in M$, the values of the elements of $S$ at $m$ span $\Delta(m)$.

A smooth generalized distribution contained in $T M$ is called a smooth tangent distribution; a smooth generalized distribution contained in $T^{*} M$ is called a smooth cotangent distribution.

\subsubsection{Smooth orthogonals and annihilators}

The Pontryagin bundle $\mathrm{P}_{M}=T M \oplus T^{*} M$ of a smooth manifold $M$ is endowed with a nondegenerate symmetric fiberwise bilinear form of signature $(\operatorname{dim} M, \operatorname{dim} M)$ given by

$$
\left\langle\left(u_{m}, \alpha_{m}\right),\left(v_{m}, \beta_{m}\right)\right\rangle:=\left\langle\beta_{m}, u_{m}\right\rangle+\left\langle\alpha_{m}, v_{m}\right\rangle
$$

for all $u_{m}, v_{m} \in T_{m} M$ and $\alpha_{m}, \beta_{m} \in T_{m}^{*} M$.

If $\Delta \subset \mathrm{P}_{M}$ is a smooth generalized distribution, its smooth orthogonal is the smooth generalized distribution $\Delta^{\perp} \subseteq \mathrm{P}_{M}$ defined by

$$
\Delta^{\perp}(m):=\left\{\tau(m) \mid \begin{array}{c}
\tau \in \Gamma\left(\mathrm{P}_{M}\right) \text { with } m \in \operatorname{Dom}(\tau) \text { is such that for all } \\
\sigma \in \Gamma(\Delta) \text { with } m \in \operatorname{Dom}(\sigma), \\
\text { we have }\langle\sigma, \tau\rangle=0 \text { on } \operatorname{Dom}(\tau) \cap \operatorname{Dom}(\sigma)
\end{array}\right\} .
$$

Note that the smooth orthogonal of a smooth generalized distribution is smooth, by construction. The inclusion $\Delta \subset \Delta^{\perp \perp}$ is, in general, strict. If the distribution $\Delta$ is a vector subbundle of $\mathrm{P}_{M}$, then its smooth orthogonal is also a vector subbundle of $\mathrm{P}_{M}$.

Let $\mathcal{T} \subseteq T M$ be a tangent distribution. The smooth annihilator of $\mathcal{T}$ is the smooth codistribution $\mathcal{T}^{\circ} \subseteq T^{*} M$ defined by

$$
\mathcal{T}^{\circ}(m):=\left\{\begin{array}{l|l}
\alpha_{m} & \begin{array}{l}
\alpha \in \Omega^{1}(M), m \in \operatorname{Dom}(\alpha) \text { and } \alpha(X)=0 \\
\text { on } \operatorname{Dom}(\alpha) \cap \operatorname{Dom}(X) \text { for all } X \in \Gamma(\mathcal{T})
\end{array}
\end{array}\right\}
$$

for all $m \in M$. Analogously, we define the smooth annihilator $\mathcal{C}^{\circ} \subseteq T M$ of a codistribution $\mathcal{C} \subseteq T^{*} M$

The tangent distribution $\mathcal{V}$ spanned by the fundamental vector fields of the action of a Lie group $G$ on a manifold $M$ is defined at every point $m \in M$ by $\mathcal{V}(m):=$ $\left\{\xi_{M}(m) \mid \xi \in \mathfrak{g}\right\}$. If the action is not free, the rank of the fibers of $\mathcal{V}$ can vary on $M$. The 
smooth annihilator $\mathcal{V}^{\circ}$ of $\mathcal{V}$ has the expression

$$
\mathcal{V}^{\circ}(m)=\left\{\alpha(m) \mid \alpha \in \Omega^{1}(M), m \in \operatorname{Dom}(\alpha), \text { such that } \alpha\left(\xi_{M}\right)=0 \text { for all } \xi \in \mathfrak{g}\right\}
$$

We will also use the smooth generalized distribution $\mathcal{K}:=\mathcal{V} \oplus\{0\} \subseteq \mathrm{P}_{M}$ and its smooth orthogonal $\mathcal{K}^{\perp}=T M \oplus \mathcal{V}^{\circ}$.

\subsubsection{Generalized foliations and integrability of tangent distributions}

To give content to the notion of integrability of a smooth tangent distribution and elaborate on it, we need to quickly review the concept and main properties of generalized foliations (see [26-29] for the original articles and [21, 24, 30], or [23], for a quick review of this theory).

A generalized foliation on $M$ is a partition $\mathfrak{F}:=\left\{\mathcal{L}_{\alpha}\right\}_{\alpha \in A}$ of $M$ into disjoint connected sets, called leaves, such that each point $m \in M$ has a generalized foliated chart $\left(U, \varphi: U \rightarrow V \subseteq \mathbb{R}^{\operatorname{dim} M}\right), m \in U$. This means that there is some natural number $p_{\alpha} \leq \operatorname{dim} M$, called the dimension of the leaf $\mathcal{L}_{\alpha}$, and a subset $S_{\alpha} \subset$ $\mathbb{R}^{\operatorname{dim} M-p_{\alpha}}$ such that $\varphi\left(U \cap \mathcal{L}_{\alpha}\right)=\left\{\left(x^{1}, \ldots, x^{\operatorname{dim} M}\right) \in V \mid\left(x^{p_{\alpha}+1}, \ldots, x^{\operatorname{dim} M}\right) \in S_{\alpha}\right\}$. Note that each $\left(x_{\circ}^{p_{\alpha}+1}, \ldots, x_{\circ}^{\operatorname{dim} M}\right) \in S_{\alpha}$ determines a connected component $\left(U \cap \mathcal{L}_{\alpha}\right) \circ$ of $U \cap \mathcal{L}_{\alpha}$, that is, $\varphi\left(\left(U \cap \mathcal{L}_{\alpha}\right)_{\circ}\right)=\left\{\left(x^{1}, \ldots, x^{p_{\alpha}}, x_{\circ}^{p_{\alpha}+1}, \ldots, x_{\circ}^{\operatorname{dim} M}\right) \in V\right\}$. The key difference with the concept of foliation is that the number $p_{\alpha}$ can change from leaf to leaf. The generalized foliated charts induce on each leaf a smooth manifold structure that makes them into initial submanifolds of $M$.

A leaf $\mathcal{L}_{\alpha}$ is called regular if it has an open neighborhood that intersects only leaves whose dimension equals $\operatorname{dim} \mathcal{L}_{\alpha}$. If such a neighborhood does not exist, then $\mathcal{L}_{\alpha}$ is called a singular leaf. A point is called regular (singular) if it is contained in a regular (singular) leaf. The set of vectors tangent to the leaves of $\mathfrak{F}$ is defined by

$$
T(M, \mathfrak{F}):=\bigcup_{\alpha \in A} \bigcup_{m \in \mathcal{L}_{\alpha}} T_{m} \mathcal{L}_{\alpha} \subset T M
$$

Let us turn now to the relationship between distributions and generalized foliations. In all that follows, $\mathcal{T}$ is a smooth tangent distribution. An integral manifold of $\mathcal{T}$ is an injectively immersed connected manifold $\iota_{L}: L \hookrightarrow M$, where $\iota_{L}$ is the inclusion, satisfying the condition $T_{m} \iota_{L}\left(T_{m} L\right) \subset \mathcal{T}(m)$, for every $m \in L$. The integral manifold $L$ is of maximal dimension at $m \in L$ if $T_{m} \iota_{L}\left(T_{m} L\right)=\mathcal{T}(m)$. The distribution $\mathcal{T}$ is completely integrable if for every $m \in M$ there is an integral manifold $L$ of $\mathcal{T}, m \in L$, everywhere of 
maximal dimension. The distribution $\mathcal{T}$ is involutive if it is invariant under the (local) flows associated to differentiable sections of $\mathcal{T}$. The distribution $\mathcal{T}$ is algebraically involutive if for any two smooth vector fields defined on an open set of $M$ which take values in $\mathcal{T}$, their bracket also takes values in $\mathcal{T}$. Clearly, involutive distributions are algebraically involutive and the converse is true if the distribution is a vector subbundle.

Recall that the Frobenius theorem states that a vector subbundle of TM is algebraically involutive if and only if it is the tangent bundle of a foliation on $M$.

The same is true for distributions under the involutivity assumption: A smooth distribution is involutive if and only if it coincides with the set of vectors tangent to a generalized foliation, that is, it is completely integrable. This is known as the StefanSussmann Theorem.

We will formulate the Stefan-Sussmann theorem in the setting of a smooth tangent distribution spanned by a family of vector fields. Note that each smooth tangent distribution is spanned by the family of its smooth sections.

Let $\mathcal{F}$ be an everywhere defined family of local vector fields on $M$. By everywhere defined, we mean that for every $m \in M$ there exists $X \in \mathcal{F}$ such that $m \in \operatorname{Dom}(X)$. Associate to the flows of the vector fields in $F$ the set of local diffeomorphisms $\mathcal{A}_{\mathcal{F}}:=$ $\left\{\phi_{t} \mid \phi_{t}\right.$ flow of $\left.X \in \mathcal{F}\right\}$ of $M$ and the pseudogroup of transformations generated by it,

$$
A_{\mathcal{F}}:=(\mathbb{I}, M) \bigcup\left\{\phi_{t_{1}}^{1} \circ \cdots \circ \phi_{t_{n}}^{n} \mid n \in \mathbb{N} \text { and } \phi_{t_{k}}^{k} \in \mathcal{A}_{\mathcal{F}} \text { or }\left(\phi_{t_{k}}^{k}\right)^{-1} \in \mathcal{A}_{\mathcal{F}} \text { for } k=1, \ldots, n\right\}
$$

Analogously, we also define, for any $z \in M$, the following vector subspace of $T_{z} M$ :

$$
\begin{aligned}
\mathcal{D}_{\mathcal{F}}(z) & :=\operatorname{span}_{\mathbb{R}}\left\{\left.\frac{\mathrm{d}}{\mathrm{d} t}\right|_{t=t_{0}} \phi_{t}(y) \mid \phi_{t} \text { flow of } X \in \mathcal{F}, \phi_{t_{0}}(y)=z\right\} \\
& =\operatorname{span}_{\mathbb{R}}\left\{X(z) \in T_{z} M \mid X \in \mathcal{F} \text { and } z \in \operatorname{Dom}(X)\right\} .
\end{aligned}
$$

Note that, by construction, $\mathcal{D}_{\mathcal{F}}$ is a smooth tangent distribution; $\mathcal{D}_{\mathcal{F}}$ is said to be the smooth tangent distribution spanned by $\mathcal{F}$.

The $A_{\mathcal{F}}$-orbits, also called the accessible sets of the family $\mathcal{F}$, form a generalized foliation (see, e.g., [23]). An important question is determining when the smooth tangent distribution $\mathcal{D}_{\mathcal{F}}$ spanned by $\mathcal{F}$ is integrable. 
Theorem 2.1 ([27, 29]). Let $\mathcal{D}_{\mathcal{F}}$ be a differentiable generalized distribution on the smooth manifold $M$ spanned pointwise by an everywhere defined family of vector fields $\mathcal{F}$. The following are equivalent:

1. The distribution $\mathcal{D}_{\mathcal{F}}$ is invariant under the pseudogroup of transformations generated by $\mathcal{F}$, that is, for each $\phi_{T} \in A_{\mathcal{F}}$ and for each $z \in M$ in the domain of $\phi_{T}$,

$$
T_{z} \phi_{T}\left(\mathcal{D}_{\mathcal{F}}(z)\right)=\mathcal{D}_{\mathcal{F}}\left(\phi_{T}(z)\right)
$$

2. The distribution $\mathcal{D}_{\mathcal{F}}$ is integrable and its maximal integral manifolds are the $A_{\mathcal{F}}$-orbits.

As already mentioned, given an involutive (and hence a completely integrable) distribution $\mathcal{T}$, each point $m \in M$ belongs to exactly one connected integral manifold $\mathcal{L}_{m}$ that is maximal relative to inclusion. It turns out that $\mathcal{L}_{m}$ is an initial submanifold and that it is also the accessible set of $m$, that is, $\mathcal{L}_{m}$ equals the subset of points in $M$ that can be reached by applying to $m$ a finite number of composition of flows of elements of $\Gamma(\mathcal{T})$. The collection of all maximal integral submanifolds of $\mathcal{T}$ forms a generalized foliation $\mathfrak{F}_{\mathcal{T}}$ such that $\mathcal{T}=T\left(M, \mathfrak{F}_{\mathcal{T}}\right)$. Conversely, given a generalized foliation $\mathfrak{F}$ on $M$, the subset $T(M, \mathfrak{F}) \subset T M$ is a smooth completely integrable (and hence involutive) distribution whose collection of maximal integral submanifolds coincides with $\mathfrak{F}$. These two statements expand the Stefan-Sussmann Theorem cited above.

\subsection{Generalities on Dirac structures}

\subsubsection{Dirac structures}

Recall that the Pontryagin bundle $\mathrm{P}_{M}=T M \oplus T^{*} M$ of a smooth manifold $M$ is endowed with a nondegenerate symmetric fiberwise bilinear form of signature $(\operatorname{dim} M, \operatorname{dim} M)$ given by (1). A Dirac structure $[8,9]$ on $M$ is a Lagrangian subbundle $\mathrm{D} \subset \mathrm{P}_{M}$. That is, $\mathrm{D}$ coincides with its orthogonal relative to (1) and so its fibers are necessarily $\operatorname{dim} M$-dimensional.

The space $\Gamma\left(T M \oplus T^{*} M\right)$ of smooth local sections of the Pontryagin bundle is also endowed with a $\mathbb{R}$-bilinear skew-symmetric bracket (which does not satisfy the 
Jacobi identity) given by

$$
\begin{aligned}
{[(X, \alpha),(Y, \beta)]: } & =\left([X, Y], £_{X} \beta-£_{Y} \alpha+\frac{1}{2} \mathrm{~d}(\alpha(Y)-\beta(X))\right) \\
& =\left([X, Y], £_{X} \beta-\mathrm{i}_{Y} \mathrm{~d} \alpha-\frac{1}{2} \mathrm{~d}\langle(X, \alpha),(Y, \beta)\rangle\right)
\end{aligned}
$$

(see [8]). The Dirac structure is closed (or integrable) if $[\Gamma(\mathrm{D}), \Gamma(\mathrm{D})] \subset \Gamma(\mathrm{D})$. Since $\langle(X, \alpha),(Y, \beta)\rangle=0$ if $(X, \alpha),(Y, \beta) \in \Gamma(\mathrm{D})$, integrability of the Dirac structure is often expressed in the literature relative to a nonskew-symmetric bracket that differs from (2) by eliminating in the second line the third term of the second component. This truncated expression which satisfies the Jacobi identity but is no longer skew-symmetric is called the Courant-Dorfman bracket:

$$
[(X, \alpha),(Y, \beta)]:=\left([X, Y], £_{X} \beta-\mathrm{i}_{Y} \mathrm{~d} \alpha\right) .
$$

A Dirac structure D on a manifold $M$ defines two smooth tangent distributions $\mathrm{G}_{0}, \mathrm{G}_{1} \subset T M$ and two smooth cotangent distributions $\mathrm{P}_{0}, \mathrm{P}_{1} \subset T^{*} M$; their fibers at $m \in M$ are:

$$
\begin{aligned}
& \mathrm{G}_{0}(m):=\left\{X(m) \in T_{m} M \mid X \in \mathfrak{X}(M),(X, 0) \in \Gamma(\mathrm{D})\right\}, \\
& \mathrm{G}_{1}(m):=\left\{X(m) \in T_{m} M \mid X \in \mathfrak{X}(M), \text { there exists } \alpha \in \Omega^{1}(M), \text { such that }(X, \alpha) \in \Gamma(\mathrm{D})\right\},
\end{aligned}
$$

and

$$
\begin{aligned}
& \mathrm{P}_{0}(m):=\left\{\alpha(m) \in T_{m}^{*} M \mid \alpha \in \Omega^{1}(M),(0, \alpha) \in \Gamma(\mathrm{D})\right\}, \\
& \mathrm{P}_{1}(m):=\left\{\alpha(m) \in T_{m}^{*} M \mid \alpha \in \Omega^{1}(M), \text { there exists } X \in \mathfrak{X}(M), \text { such that }(X, \alpha) \in \Gamma(\mathrm{D})\right\} .
\end{aligned}
$$

The smoothness of $G_{0}, G_{1}, P_{0}$, and $P_{1}$ is obvious since, by definition, they are generated by smooth local sections. In general, these are not vector subbundles of $T M$ and $T^{*} M$, respectively. It is also clear that $G_{0} \subset G_{1}$ and $P_{0} \subset P_{1}$. In general, $P_{0}=G_{1}^{\circ}, G_{0}=P_{1}^{\circ}, P_{1} \subseteq$ $\mathrm{G}_{0}^{\circ}$, and $\mathrm{G}_{1} \subseteq \mathrm{P}_{0}^{\circ}$. If $\mathrm{P}_{1}$ (respectively, $\mathrm{G}_{1}$ ) has constant rank on $M$, the first (respectively, second) inclusion is an equality.

A function $f \in C^{\infty}(M)$ is called D-admissible, or simply admissible if there is no possibility of confusion, if there exists a vector field $X \in \mathfrak{X}(M)$ such that $(X, \mathrm{~d} f) \in \Gamma(\mathrm{D})$. 
The section $(X, \mathrm{~d} f)$ is then called (D-) admissible or (D-)Hamiltonian and $X=: X_{f}$ is a (D-)Hamiltonian vector field for $f$.

Note that the vector field $X_{f}$ is not unique, if $\mathrm{G}_{0} \neq\{0\}$; if $X_{f}$ is a Hamiltonian vector field for $f$, then, for any section $Z$ of $\mathrm{G}_{0}$, the sum $X_{f}+Z$ is also a Hamiltonian vector field for $f$. Indeed, since $\left(X_{f}, \mathrm{~d} f\right),(Z, 0) \in \Gamma(\mathrm{D})$, the sum $\left(X_{f}+Z, \mathrm{~d} f\right)=\left(X_{f}, \mathrm{~d} f\right)+(Z, 0)$ is also a section of $D$. The smooth tangent distribution $G_{0}$ is spanned by the Hamiltonian vector fields of the constant functions, which are consequently all admissible.

Define a bracket $\{\cdot, \cdot\}_{\mathrm{D}}$ on the set $C^{\infty}(M)^{\mathrm{D}}$ of admissible functions by $\{f, g\}_{\mathrm{D}}:=$ $X_{f}(g)=-X_{g}(f)$ for all $f, g \in C^{\infty}(M)^{\mathrm{D}}$. This bracket does not depend on the choices made for $X_{g}$ and $X_{f}$, and if the Dirac manifold $(M, \mathrm{D})$ is integrable, $\{\cdot, \cdot\}_{\mathrm{D}}: C^{\infty}(M)^{\mathrm{D}} \times$ $C^{\infty}(M)^{\mathrm{D}} \rightarrow C^{\infty}(M)^{\mathrm{D}}$ is a Poisson bracket on $C^{\infty}(M)^{\mathrm{D}}$.

\subsubsection{Properties of integrable Dirac structures}

Assume that ( $M, \mathrm{D})$ is an integrable Dirac manifold. Then, relative to the Courant bracket (3) and the anchor $\pi_{T M}: \mathrm{D} \rightarrow T M$ given by the projection on the first factor, $\mathrm{D}$ becomes a Lie algebroid over $M$. The smooth distribution $\mathrm{G}_{1}=\pi_{T M}(\mathrm{D}) \subset T M$ is then completely integrable in the sense of Stefan and Sussmann and each leaf $N$ of $\mathrm{G}_{1}$ inherits a presymplectic form $\omega_{N}$ given by

$$
\omega_{N}(\tilde{X}, \tilde{Y})(p)=\alpha(Y)(p)=-\beta(X)(p)
$$

for all $p \in N$ and $\tilde{X}, \tilde{Y} \in \mathfrak{X}(N)$, where $X, Y \in \Gamma\left(\mathrm{G}_{1}\right)$ are arbitrary sections $\iota_{N}$-related to $\tilde{X}$ and $\tilde{Y}$, respectively, and $\iota_{N}: N \hookrightarrow M$ is the inclusion; $\iota_{N}$-relatedness is denoted by $\tilde{X} \sim_{\iota_{N}}$ $X, \tilde{Y} \sim_{\iota_{N}} Y$. The 1 -forms $\alpha, \beta \in \Omega^{1}(M)$ are such that $(X, \alpha),(Y, \beta) \in \Gamma(\mathrm{D})$. Formula (4) is independent of all the choices involved. Note that there is an induced Dirac structure

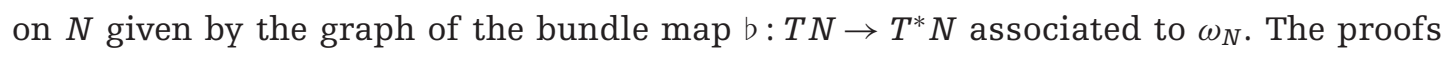
of these facts can be found in [8]. Since $\mathrm{G}_{1}$ has constant rank on $N$, the codistribution $\mathrm{P}_{0}$ has also constant rank on $N$ and $\mathrm{D} \cap\left(\left.T N \oplus T^{*} M\right|_{N}\right)$ is a smooth vector bundle over $N$. Then the induced Dirac structure $D_{N}$ on $N$ can be described in the following way:

$$
\Gamma\left(\mathrm{D}_{N}\right):=\left\{(\tilde{X}, \tilde{\alpha}) \mid \exists(X, \alpha) \in \Gamma(\mathrm{D}) \text { such that } \tilde{X} \sim_{\iota_{N}} X \text { and } \tilde{\alpha}=\iota_{N}^{*} \alpha\right\}
$$

(see [8] or [16]). 
Let $(M, \mathrm{D})$ be a closed Dirac manifold and $f \in C^{\infty}(M)^{\mathrm{D}}$ a smooth admissible function. Let $\phi$ be the flow of a corresponding Hamiltonian vector field $X_{f}$. Since

$$
\left(£_{X_{f}} X, £_{X_{f}} \alpha\right)=\left[\left(X_{f}, \mathrm{~d} f\right),(X, \alpha)\right] \in \Gamma(\mathrm{D})
$$

for all $(X, \alpha) \in \Gamma(\mathrm{D})$, we also have $\left(\phi_{t}^{*} X, \phi_{t}^{*} \alpha\right) \in \Gamma(\mathrm{D})$, for all $(X, \alpha) \in \Gamma(\mathrm{D})$. (This implication is standard, but the proof is difficult to find in the literature. It is carried out in $[13,14]$.) We get the following theorem (see also [8, Theorem 2.4.1]):

Theorem 2.2. Let ( $M, \mathrm{D})$ be a closed Dirac manifold, $f \in C^{\infty}(M)$ an admissible function of (M, D), and $X_{f}$ a Hamiltonian vector field for $f$. Let $\phi$ be the flow of $X_{f}$. The Dirac structure is invariant under $\phi_{t}$ for all $t$ for which $\phi_{t}$ is defined, that is, $\left(\phi_{t}^{*} X, \phi_{t}^{*} \alpha\right) \in \Gamma$ (D) for all $(X, \alpha) \in \Gamma(\mathrm{D})$.

Thus, if $g \in C^{\infty}(M)^{\mathrm{D}}$ is an admissible function and $\phi$ is the flow of the vector field $X_{f}$, then the function $\phi_{t}^{*} g$ is also admissible. Furthermore, if $X_{H}$ is a solution of the implicit Hamiltonian system $\left(X_{H}, \mathrm{~d} H\right) \in \Gamma$ (D) for a Hamiltonian $H \in C^{\infty}(M)^{\mathrm{D}}$, then the Dirac structure $D$ is conserved along the solution curves of the system.

Note that if $X$ is an arbitrary section of $\mathrm{G}_{1}$, it is not possible to show in the same manner that the Dirac structure is conserved along the flow lines of $X$. Recall also that the space of sections of $\mathrm{G}_{1}$ is not necessarily generated by $\left\{X_{f} \mid f\right.$ admissible $\}$. Therefore, the flows of sections of $\mathrm{G}_{1}$ do not conserve the Dirac structure of $M$, in general. As we shall see later on, this is a major technical problem. Certain conditions on the admissible functions will have to be imposed.

\subsection{Proper actions and orbit-type manifolds}

\subsubsection{The stratification by orbit types}

In this section, we consider a smooth and proper action

$$
\begin{aligned}
\Phi: G \times M & \rightarrow M, \\
(g, m) & \mapsto \Phi(g, m)=\Phi_{g}(m)=g m=g \cdot m
\end{aligned}
$$

of a Lie group $G$ on a manifold $M$. Let $\pi: M \rightarrow \bar{M}:=M / G$ be the orbit map. 
For each closed Lie subgroup $H$ of $G$, we define the isotropy type set

$$
M_{H}=\left\{m \in M \mid G_{m}=H\right\},
$$

where $G_{m}=\{g \in G \mid g m=m\}$ is the isotropy subgroup of $m \in M$. Since the action is proper, all isotropy groups are compact. The sets $M_{H}$, where $H$ ranges over the closed Lie subgroups of $G$ for which $M_{H}$ is nonempty, form a partition of $M$ and therefore they are the equivalence classes of an equivalence relation in $M$. Define the normalizer of $H$ in $G$ by

$$
N(H):=\left\{g \in G \mid g H g^{-1}=H\right\}
$$

$N(H)$ is a closed Lie subgroup of $G$. Since $H$ is a normal subgroup of $N(H)$ the quotient $N(H) / H$ is a Lie group. If $m \in M_{H}$, we have $G_{m}=H$ and $G_{g m}=g H g^{-1}$ for all $g \in G$. Consequently, $g m \in M_{H}$ if and only if $g \in N(H)$. The action of $G$ on $M$ restricts to an action of $N(H)$ on $M_{H}$, which induces a free and proper action of $N(H) / H$ on $M_{H}$.

Define the orbit type set

$$
M_{(H)}:=\left\{m \in M \mid G_{m} \text { is conjugated to } H\right\} .
$$

Then,

$$
M_{(H)}=\left\{g m \mid g \in G, m \in M_{H}\right\}=\pi^{-1}\left(\pi\left(M_{H}\right)\right) .
$$

The connected components of $M_{H}$ and $M_{(H)}$ are embedded submanifolds of $M$; therefore, $M_{H}$ is called an isotropy-type manifold and $M_{(H)}$ an orbit-type manifold. Moreover,

$$
\pi\left(M_{(H)}\right)=\left\{g m \mid m \in M_{H}\right\} / G=M_{H} / N(H)=M_{H} /(N(H) / H)
$$

Since the action of $N(H) / H$ on $M_{H}$ is free and proper, it follows that $M_{H} /(N(H) / H)$ is a quotient manifold of $M_{H}$. Hence, the subset $\pi\left(M_{(H)}\right) \subseteq \bar{M}=M / G$ is a manifold.

The partitions of $M$ by the connected components of the orbit-type manifolds is a decomposition of the differential space $M$. The corresponding stratification of $M$ is called the orbit-type stratification of $M[11,24]$. The orbit space $\bar{M}=M / G$ with its quotient topology has also the structure of a stratified space with strata the projections of the connected components of the orbit-type manifolds. 


\subsubsection{Descending sections of $\mathrm{P}_{M}$}

Let $\mathcal{V}_{G}^{\circ}$ be the cotangent distribution on $M$ spanned pointwise by the $G$-equivariant sections of $\mathcal{V}^{\circ}$, that is, $\mathcal{V}_{G}^{\circ}(m):=\left\{\alpha_{m} \mid \alpha \in \Gamma\left(\mathcal{V}^{\circ}\right)^{G}\right\}=\left\{\mathrm{d} f(m) \mid f \in C^{\infty}(M)^{G}\right\}$ (see [18, Lemma 5.8]). If $\alpha \in \Gamma\left(\mathcal{V}^{\circ}\right)^{G}$, it pushes-forward to the "1-form" $\bar{\alpha}:=\pi_{*} \alpha$ such that, for every $\bar{Y} \in \mathfrak{X}(\bar{M})$ and every vector field $Y \in \mathfrak{X}(M)$ satisfying $Y \sim_{\pi} \bar{Y}$, we have

$$
\pi^{*}(\bar{\alpha}(\bar{Y}))=\alpha(Y)
$$

Each vector field $X$ satisfying $[X, \Gamma(\mathcal{V})] \subseteq \Gamma(\mathcal{V})$ can be written as $X=X^{G}+X^{\mathcal{V}}$, with $X^{G} \in \mathfrak{X}(M)^{G}$ and $X^{\mathcal{V}} \in \Gamma(\mathcal{V})$, and $X$ pushes-forward to a "vector field" $\bar{X}$ on $\bar{M}$. Since we will not need these objects in the rest of the paper, we will not give more details about what we call the "vector fields" and "1-forms" on the stratified space $\bar{M}=M / G$ and refer to [18] for more information.

A local section $(X, \alpha)$ of $T M \oplus \mathcal{V}^{\circ}=\mathcal{K}^{\perp}$ satisfying $[X, \Gamma(\mathcal{V})] \subseteq \Gamma(\mathcal{V})$ and $\alpha \in \Gamma\left(\mathcal{V}^{\circ}\right)^{G}$ is called a descending section of $\mathrm{P}_{M}$.

Let $\mathcal{T}$ be the tangent distribution on $M$ spanned pointwise by the vector fields that descend to $\bar{M}:=M / G$, that is, $\mathcal{T}$ is the distribution spanned pointwise by

$$
\begin{aligned}
F & :=\left\{X \in \mathfrak{X}(M) \mid X=X^{G}+X^{\mathcal{V}}, \text { with } X^{G} \in \mathfrak{X}(M)^{G} \text {, and } X^{\mathcal{V}} \in \Gamma(\mathcal{V})\right\} \\
& =\{X \in \mathfrak{X}(M) \mid[X, \Gamma(\mathcal{V})] \subseteq \Gamma(\mathcal{V})\} .
\end{aligned}
$$

We have shown in [18] that $\mathcal{T}$ is integrable and its leaves are the connected components of the orbit-type manifolds. In the same manner, let $\mathcal{T}_{G}$ be the distribution on $M$ spanned by the set $F_{G}$ of $G$-invariant vector fields on $M$ :

$$
F_{G}=\left\{X \in \mathfrak{X}(M) \mid \Phi_{g}^{*} X=X \quad \forall g \in G\right\} .
$$

It is shown in [23] that this smooth distribution is also completely integrable in the sense of Stefan and Sussmann; its leaves are the connected components of the isotropytype manifolds. Note that the integrability of $\mathcal{T}$ follows from the integrability of $\mathcal{T}_{G}$ and Proposition 3.4.6 in [23]. In particular, both distributions coincide in the case of a free action. This is why the constructions made here simplify in a significant manner in this special case, which is reviewed in Section 3.

By the considerations above, the generalized distribution $\mathcal{T} \oplus \mathcal{V}_{G}^{\circ}$ is the generalized distribution spanned pointwise by the descending sections of $\mathrm{P}_{M}$. The descending 
sections of a $G$-invariant dirac structure $D$ will be of great importance in the rest of this paper. These sections necessarily lie in $\mathrm{D} \cap\left(\mathcal{T} \oplus \mathcal{V}_{G}^{\circ}\right)$.

\subsubsection{Review of standard Dirac reduction}

Symmetries of Dirac manifolds. Let ( $M$, D) be a smooth Dirac manifold. Let $G$ be a Lie group and $\Phi: G \times M \rightarrow M$ a smooth left action. Then $G$ is called a symmetry Lie group of $\mathrm{D}$ if, for every $g \in G$, the condition $(X, \alpha) \in \Gamma(\mathrm{D})$ implies that $\left(\Phi_{g}^{*} X, \Phi_{g}^{*} \alpha\right) \in \Gamma(\mathrm{D})$. We say then that the Lie group $G$ acts canonically or by Dirac actions on $M$.

Let $\mathfrak{g}$ be a Lie algebra and $\xi \in \mathfrak{g} \mapsto \xi_{M} \in \mathfrak{X}(M)$ be a smooth left Lie algebra action, that is, the $\operatorname{map}(x, \xi) \in M \times \mathfrak{g} \mapsto \xi_{M}(x) \in T M$ is smooth and $\xi \in \mathfrak{g} \mapsto \xi_{M} \in \mathfrak{X}(M)$ is a Lie algebra anti-homomorphism. The Lie algebra $\mathfrak{g}$ is said to be a symmetry Lie algebra of $\mathrm{D}$ if for every $\xi \in \mathfrak{g}$ the condition $(X, \alpha) \in \Gamma(\mathrm{D})$ implies that $\left(£_{\xi_{M}} X, €_{\xi_{M}} \alpha\right) \in \Gamma(\mathrm{D})$. Of course, if $\mathfrak{g}$ is the Lie algebra of the Lie group $G$ and $\xi_{M}$ is the infinitesimal generator for all $\xi \in \mathfrak{g}$, then if $G$ is a symmetry Lie group of $D$ it follows that $\mathfrak{g}$ is a symmetry Lie algebra of $D$.

Standard Dirac reduction. We present a short review of the Dirac reduction methods. For more details, see $[18,19]$. Let $(M, D)$ be a smooth Dirac manifold acted upon in a smooth proper and Dirac manner by a Lie group $G$ such that the intersection $\mathrm{D} \cap\left(\mathcal{T} \oplus \mathcal{V}_{G}^{\circ}\right)$ is spanned pointwise by its descending sections.

Consider the subset $\mathcal{D}^{G}$ of $\Gamma(\mathrm{D})$ defined by

$$
\mathcal{D}^{G}:=\left\{(X, \alpha) \in \Gamma(\mathrm{D}) \mid \alpha \in \Gamma\left(\mathcal{V}^{\circ}\right)^{G} \text { and }[X, \Gamma(\mathcal{V})] \subseteq \Gamma(\mathcal{V})\right\},
$$

that is, the set of the descending sections of $D$.

Each vector field $X$ satisfying $[X, \Gamma(\mathcal{V})] \subseteq \Gamma(\mathcal{V})$ pushes forward to a vector field $\bar{X}$ on $\bar{M}$. For each stratum $\bar{P}$ of $\bar{M}$, the restriction of $\bar{X}$ to points of $\bar{P}$ is a vector field $X_{\bar{P}}$ on $\bar{P}$. On the other hand, if $(X, \alpha) \in \mathcal{D}^{G}$, then we have $\alpha \in \Gamma\left(\mathcal{V}^{\circ}\right)^{G}$ and it pushes forward to the 1 -form $\bar{\alpha}:=\pi_{*} \alpha$ such that, for every $\bar{Y} \in \mathfrak{X}(\bar{M})$ and every vector field $Y \in \mathfrak{X}(M)$ satisfying $Y \sim_{\pi} \bar{Y}$, we have

$$
\pi^{*}(\bar{\alpha}(\bar{Y}))=\alpha(Y)
$$

Moreover, for each stratum $\bar{P}$ of $\bar{M}$, the restriction of $\bar{\alpha}$ to points of $\bar{P}$ defines a 1 -form $\alpha_{\bar{P}}$ on $\bar{P}$. Let

$$
\overline{\mathcal{D}}:=\left\{(\bar{X}, \bar{\alpha}) \mid(X, \alpha) \in \mathcal{D}^{G}\right\}
$$


and for each stratum $\bar{P}$ of $\bar{M}$, set

$$
\mathcal{D}_{\bar{P}}:=\left\{\left(X_{\bar{P}}, \alpha_{\bar{P}}\right) \mid(\bar{X}, \bar{\alpha}) \in \overline{\mathcal{D}}\right\} .
$$

Define the smooth generalized distribution $\mathrm{D}_{\bar{P}}$ on $\bar{P}$ by

$$
\mathrm{D}_{\bar{P}}(s):=\left\{\left(X_{\bar{P}}(s), \alpha_{\bar{P}}(s)\right) \in T_{s} \bar{P} \times T_{s}^{*} \bar{P} \mid\left(X_{\bar{P}}, \alpha_{\bar{P}}\right) \in \mathcal{D}_{\bar{P}}\right\}
$$

for all $s \in \bar{P}$. Note that $\Gamma\left(\mathrm{D}_{\bar{P}}\right)=\mathcal{D}_{\bar{P}}$. We have the following theorem.

Theorem 2.3 ([18]). Let (M, D) be a Dirac manifold with a proper Dirac action of a connected Lie group $G$ on it. Let $\bar{P}$ be a stratum of the quotient space $\bar{M}$. If $\mathrm{D} \cap\left(\mathcal{T} \oplus \mathcal{V}_{G}^{\circ}\right)$ is spanned pointwise by its descending sections, then $D_{\bar{P}}$ defined in (8) is a Dirac structure on $\bar{P}$. If $(M, \mathrm{D})$ is integrable, then $\left(\bar{P}, \mathrm{D}_{\bar{P}}\right)$ is integrable.

In the regular case, this simplifies to the following statement.

Theorem 2.4 ([19]). Let $G$ be a connected Lie group acting in a proper way on the manifold $M$ such that all isotropy subgroups are conjugated. Assume that $\mathrm{D} \cap \mathcal{K}^{\perp}$ has constant rank on $M$, where $\mathcal{K}^{\perp}:=T M \oplus \mathcal{V}^{\circ}$. Then the Dirac structure D on $M$ induces a Dirac structure $\overline{\mathrm{D}}$ on the quotient $\bar{M}=M / G$ given by

$$
\overline{\mathrm{D}}(\bar{m}):=\left\{(\bar{X}(\bar{m}), \bar{\alpha}(\bar{m})) \in T_{\bar{m}} \bar{M} \times T_{\bar{m}}^{*} \bar{M} \mid \begin{array}{c}
\exists X \in \mathfrak{X}(M) \text { such that } X \sim_{\pi} \bar{X} \\
\text { and }\left(X, \pi^{*} \bar{\alpha}\right) \in \Gamma(\mathrm{D})
\end{array}\right\},
$$

for all $\bar{m} \in \bar{M}$. If $\mathrm{D}$ is integrable, then $\overline{\mathrm{D}}$ is also integrable.

\section{The Free Case}

In this section, we present, without proofs, the theory of optimal reduction for free Lie group actions. We do this because the main ideas are easier to follow in this situation and because this case follows closely the nonfree Poisson case. The proofs will be given later for the general case of a proper action; this is technically considerably more involved due to the fact that the characteristic distribution $\mathrm{P}_{1}$ of an arbitrary Dirac structure is not equal to $T^{*} M$ (as was the case for a Poisson structure). In the general case, two types of natural optimal distributions arise simultaneously as generalizations 
of the Poisson optimal distribution. This is related to the fact that one can consider orbittype and isotropy-type manifolds when carrying out singular reduction. In addition, as we shall see, these two natural distributions are not integrable, in general. In the free case, where they are both equal, one has to assume that the intersection of the Dirac structure with the vector bundle $\mathcal{K}^{\perp}$ associated to the action has constant rank (as for the standard reduction). In the general case, this does not make sense because $\mathcal{K}^{\perp}$ is not necessarily smooth; we will give additional hypotheses guaranteeing the integrability of the optimal distributions.

Let $(M, D)$ be a closed Dirac manifold, $G$ a symmetry Lie group of $\mathrm{D}$ acting freely and properly on $M$. Assume in the following that $\mathrm{D} \cap \mathcal{K}^{\perp}$ is a vector bundle, where $\mathcal{K}=$ $\mathcal{V} \oplus\{0\} \subset T M \oplus T^{*} M$ and $\mathcal{K}^{\perp}=T M \oplus \mathcal{V}^{\circ}$. To define the optimal momentum map (as in [23, $\S 5.5 .7])$ we need to introduce an additional smooth distribution. Define

$\mathcal{D}_{G}(m):=\left\{X(m) \mid\right.$ there is $\alpha \in \Gamma\left(\mathcal{V}^{\circ}\right) \subseteq \Omega^{1}(M)$ such that $\left.(X, \alpha) \in \Gamma(\mathrm{D})\right\} \subseteq \mathrm{G}_{1}(m)$,

for all $m \in M$. Then $\mathcal{D}_{G}=\bigcup_{m \in M} \mathcal{D}_{G}(m)$ is a smooth distribution on $M$.

If the manifold $M$ is Poisson and the Dirac structure is the graph of the Poisson map $\sharp: T^{*} M \rightarrow T M$, then $\mathcal{D}_{G}(p)=\left\{X_{f}(p) \mid\right.$ there is $f \in C^{\infty}(M)^{G}$ such that $\left.X_{f}=\sharp(d)\right\}$, which recovers the definition in [23].

Returning to the general case of Dirac manifolds, note that

$$
\mathcal{D}_{G}=\pi_{T M}\left(\mathrm{D} \cap\left(T M \oplus \mathcal{V}^{\circ}\right)\right)=\pi_{T M}\left(\mathrm{D} \cap \mathcal{K}^{\perp}\right),
$$

where $\pi_{T M}: T M \oplus T^{*} M \rightarrow T M$ is the projection on the first factor and that we always have

$$
\mathrm{G}_{0} \subseteq \mathcal{D}_{G} \subseteq \mathrm{G}_{1}
$$

We have the following lemmas.

Lemma 3.1. Let $(X, \alpha),(Y, \beta) \in \Gamma\left(\mathrm{D} \cap \mathcal{K}^{\perp}\right)$, that is, $X, Y \in \Gamma\left(\mathcal{D}_{G}\right)$. Then the 1-form $£_{X} \beta-$ $\mathrm{i}_{Y} \mathrm{~d} \alpha$ is a local section of $\mathcal{V}^{\circ}$.

For the proof see Lemma 4.2.

Corollary 3.2. If $D$ is integrable, the space of local sections of the intersection of vector bundles $\mathrm{D} \cap \mathcal{K}^{\perp}$ is closed under the Courant bracket. Hence, under the assumption 
that $\mathrm{D} \cap \mathcal{K}^{\perp}$ has constant dimensional fibers, this vector bundle inherits a Lie algebroid structure relative to the Courant-Dorfman bracket on $\Gamma\left(\mathrm{D} \cap \mathcal{K}^{\perp}\right)$ and the anchor map $\pi_{T M}: \mathrm{D} \cap \mathcal{K}^{\perp} \rightarrow T M$. Thus, the distribution $\mathcal{D}_{G}=\pi_{T M}\left(\mathrm{D} \cap \mathcal{K}^{\perp}\right)$ is integrable in the sense of Stefan-Sussmann.

This corollary is an immediate consequence of Lemma 3.1 and the closedness of $\mathrm{D}$. Note that in the general case of proper nonfree actions, we will have to assume that the corresponding distributions are integrable, or give additional hypotheses under which this is true.

Thus, if $\mathrm{D} \cap \mathcal{K}^{\perp}$ is a vector bundle, it is a Lie algebroid and $M$ admits hence a generalized foliation by the leaves of the generalized distribution $\mathcal{D}_{G}$ (see [8]). The optimal momentum map is now defined like in [23].

Definition 3.3. Assume that $\mathrm{D} \cap \mathcal{K}^{\perp}$ is a vector subbundle of $T M \oplus T^{*} M$. The projection

$$
\mathcal{J}: M \rightarrow M / \mathcal{D}_{G}
$$

on the leaf space of $\mathcal{D}_{G}$ is called the (Dirac) optimal momentum map.

In order to formulate the reduction theorem for the optimal momentum map, we need an induced action of $G$ on the leaf space of $\mathcal{D}_{G}$. This does not follow, as usual, from the $G$-equivariance of the vector fields spanning $\mathcal{D}_{G}$ because, in this case, they are not necessarily $G$-equivariant.

Proposition 3.4. If $m$ and $m^{\prime}$ are in the same leaf of $\mathcal{D}_{G}$ then $\Phi_{g}(m)$ and $\Phi_{g}\left(m^{\prime}\right)$ are in the same leaf of $\mathcal{D}_{G}$ for all $g \in G$. Hence there is a well-defined action $\bar{\Phi}: G \times M / \mathcal{D}_{G} \rightarrow M / \mathcal{D}_{G}$ given by

$$
\bar{\Phi}_{g}(\mathcal{J}(m)):=\mathcal{J}\left(\Phi_{g}(m)\right)
$$

For the proof, see Propositions 5.1 and 5.3.

Denote by $G_{\rho}$ the isotropy subgroup of $\rho \in M / \mathcal{D}_{G}$ for this induced action. If $g \in G_{\rho}$ and $m \in \mathcal{J}^{-1}(\rho)$, then

$$
\mathcal{J}\left(\Phi_{g}(m)\right)=\bar{\Phi}_{g}(\mathcal{J}(m))=\bar{\Phi}_{g}(\rho)=\rho=\mathcal{J}(m)
$$


and we conclude, as usual, that $G_{\rho}$ leaves $\mathcal{J}^{-1}(\rho)$ invariant. Thus, we get an induced action of $G_{\rho}$ on $\mathcal{J}^{-1}(\rho)$, which is free if the original $G$-action on $M$ is free.

Also, $\mathcal{J}^{-1}(\rho)$ is an initial submanifold of $M$ since it is a leaf of the generalized foliation defined by the integrable distribution $\mathcal{D}_{G}$. By Ortega and Ratiu [23, Proposition 3.4.4], there is a unique smooth structure on $G_{\rho}$ with respect to which this subgroup is an initial Lie subgroup of $G$ with Lie algebra

$$
\mathfrak{g}_{\rho}=\left\{\xi \in \mathfrak{g} \mid \xi_{M}(m) \in T_{m} \mathcal{J}^{-1}(\rho) \text {, for all } m \in \mathcal{J}^{-1}(\rho)\right\}
$$

In general, $G_{\rho}$ is not closed in $G$.

Definition 3.5. Let ( $M, \mathrm{D})$ be a Dirac manifold with integrable Dirac structure D and $G$ a Lie group acting canonically on it. Let $P$ be a set and $\mathbf{J}: M \rightarrow P$ a map. We say that $\mathbf{J}$ has the Noether property for the $G$-action on $(M, D)$ if the flow $F_{t}$ of any implicit Hamiltonian vector field associated to any $G$-invariant admissible function $h \in C^{\infty}(M)$ preserves the fibers of $\mathbf{J}$, that is,

$$
\mathbf{J} \circ F_{t}=\left.\mathbf{J}\right|_{\operatorname{Dom}\left(F_{t}\right)},
$$

where $\operatorname{Dom}\left(F_{t}\right)$ is the domain of definition of $F_{t}$.

Like in the Poisson case (see [23, Theorem 5.5.15]), one gets the following universality property. Note that if $D \cap \mathcal{K}^{\perp}$ is spanned by sections with exact cotangent projections, that is, by the family $\left\{\left(X_{f}, \mathrm{~d} f\right) \in \Gamma(\mathrm{D}) \mid \mathrm{d} f \in \Gamma\left(\mathcal{V}^{\circ}\right)\right\}$, then, by $G$-invariant averaging, it is spanned by $\left\{\left(X_{f}, \mathrm{~d} f\right) \in \Gamma(\mathrm{D}) \mid f \in C^{\infty}(M)^{G}\right\}$.

Theorem 3.6. Let $G$ be a symmetry Lie group of the Dirac manifold ( $M, \mathrm{D})$ and $\mathrm{J}: M \rightarrow P$ a function with the Noether property. Assume that $\mathrm{D} \cap \mathcal{K}^{\perp}$ is spanned by sections with exact cotangent projections. Then there exists a unique map $\phi: M / \mathcal{D}_{G} \rightarrow P$ such that the following diagram commutes:

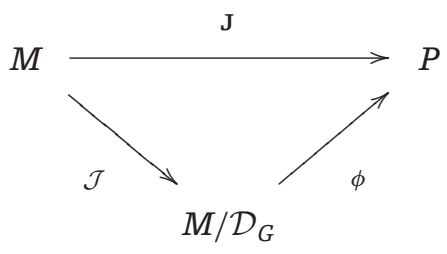


If $\mathbf{J}$ is $G$-equivariant with respect to some $G$-action on $P$, then $\phi_{G}$ is also $G$-equivariant. If $\mathbf{J}$ is smooth and $M / \mathcal{D}_{G}$ is a smooth manifold, then $\phi_{G}$ is also smooth.

For the proof, see Theorem 5.6.

Now we can generalize the optimal reduction procedure from Poisson manifolds (see [23, Theorem 9.1.1]) to closed Dirac manifolds. As we shall see, with appropriately extended definitions this important desingularization method works also for Dirac manifolds.

Theorem 3.7 (Optimal point reduction by Dirac actions). Let ( $M, \mathrm{D})$ be an integrable Dirac manifold and $G$ a Lie group acting freely and properly on $M$ and leaving the Dirac structure invariant. Assume that $\mathrm{D} \cap \mathcal{K}^{\perp}$ is constant dimensional and let $\mathcal{J}: M \rightarrow M / \mathcal{D}_{G}$ be the optimal (Dirac) momentum map associated to this action. Then, for any $\rho \in M / \mathcal{D}_{G}$ whose isotropy subgroup $G_{\rho}$ acts properly on $\mathcal{J}^{-1}(\rho)$, the orbit space $M_{\rho}=\mathcal{J}^{-1}(\rho) / G_{\rho}$ is a smooth presymplectic regular quotient manifold with presymplectic form $\omega_{\rho} \in \Omega^{2}\left(M_{\rho}\right)$ defined by

$$
\left(\pi_{\rho}^{*} \omega_{\rho}\right)(m)(X(m), Y(m))=\alpha_{m}(Y(m))=-\beta_{m}(X(m))
$$

for any $m \in \mathcal{J}^{-1}(\rho)$ and any $X, Y \in \Gamma\left(\mathcal{D}_{G}\right)$ defined on an open set containing $m$, where $\alpha, \beta \in \Gamma\left(\mathcal{V}^{\circ}\right)$ are such that $(X, \alpha),(Y, \beta) \in \Gamma\left(\mathrm{D} \cap \mathcal{K}^{\perp}\right)$, and $\pi_{\rho}: \mathcal{J}^{-1}(\rho) \rightarrow M_{\rho}$ is the projection. The pair $\left(M_{\rho}, \mathrm{D}_{\rho}\right)$ is called the (Dirac optimal) point reduced space of $(M, \mathrm{D})$ at $\rho$, where $D_{\rho}$ is the graph of the presymplectic form $\omega_{\rho}$.

Note that if $\mathrm{D}$ is the graph of a Poisson structure on $M$, the distribution $\mathrm{G}_{0}$ is $\{0\}$, all functions in $C^{\infty}(M)$ are admissible, and we are in the setting of the optimal point reduction by Poisson actions (see [23, Theorem 9.1.1]).

Recall that, since $\mathrm{D} \cap \mathcal{K}^{\perp}$ is assumed to have constant dimensional fibers, one can build the reduced Dirac manifold $(\bar{M}, \bar{D})$ as in Theorem 2.4. The following theorem gives the relation between the reduced manifold $\bar{M}$ and the reduced manifolds $M_{\rho}$ given by the optimal reduction theorem.

Theorem 3.8. If $m \in \mathcal{J}^{-1}(\rho) \subseteq M$, the reduced manifold $M_{\rho}$ is diffeomorphic to the presymplectic leaf $\bar{N}$ through $\pi(m)$ of the reduced Dirac manifold ( $\bar{M}, \mathrm{D}_{\text {red }}$ ) via the map $\Theta: M_{\rho} \rightarrow \bar{N}, \pi_{\rho}(x) \mapsto\left(\pi \circ i_{\rho}\right)(x)$. Furthermore, $\Theta^{*} \omega_{\bar{N}}=\omega_{\rho}$, where $\omega_{\bar{N}}$ is the presymplectic form on $\bar{N}$. 
Example 3.9. Consider a closed Dirac manifold ( $M, \mathrm{D})$ and the trivial Lie group $G=\{e\}$. Then the trivial action of $G$ on $M$ is Dirac and its vertical space is just the zero section in $T M$. Thus, the intersection $\mathrm{D} \cap \mathcal{K}^{\perp}$ is equal to $\mathrm{D} \cap\left(T M \oplus T^{*} M\right)=\mathrm{D}$. The projection $\pi_{T M}$ of $D \cap \mathcal{K}^{\perp}$ is hence just the smooth distribution $\mathrm{G}_{1}$, which is known to be completely integrable in the sense of Stefan and Sussmann (see [8]).

In this situation, we get consequently the leaves of the presymplectic foliation of the Dirac manifold ( $M, \mathrm{D}$ ) as reduced spaces and we recover also the statement of the preceding theorem in the case of a trivial action.

In the following, we will show how these results generalize to the nonfree case. The main difficulty is the fact that the distribution $\mathcal{K}$ does not have constant rank in this case, and $\mathcal{K}$ and $\mathcal{K}^{\perp}$ are not necessarily spanned by sections that descend to the quotient. Also, the sets spanned by the pairs of vector fields and 1-forms that descend to the quotient, and by the vector fields and 1-forms that descend to the quotient and are $G$-invariant, are different, in general. Taking the intersection of the Dirac structures with each one of them, yield two different singular foliations. These foliations are related to the stratifications by orbit types and by isotropy types, respectively.

\section{The Optimal Distributions}

From now on, we assume that ( $M, \mathrm{D})$ is an integrable Dirac manifold and $G$ a symmetry Lie group of $\mathrm{D}$ acting properly on $M$.

Recall from Section 2.3.2, the definitions of $\mathcal{T}$ and $\mathcal{V}_{G}^{\circ}$. In the free case, $\mathrm{D} \cap(\mathcal{T} \oplus$ $\left.\mathcal{V}_{G}^{\circ}\right)=\mathrm{D} \cap \mathcal{K}^{\perp}$, which is assumed to be of constant rank. Since in the general case, $\mathcal{T} \oplus \mathcal{V}_{G}^{\circ}$ does not have constant rank, it does not make much sense to assume that its intersection with $\mathrm{D}$ has constant rank. In the first subsection, we assume that $\mathrm{D} \cap\left(\mathcal{T} \oplus \mathcal{V}_{G}^{\circ}\right)$ is spanned by its descending sections, as is required for the standard singular reduction (see Theorem 2.3). We show that, under this hypothesis, the distributions are both algebraically involutive and the integrability of $\mathcal{D}_{G}$ follows from the integrability of $\mathcal{D}$. In the second subsection, we conclude the integrability of $\mathcal{D}_{G}$ from an "exactness" condition. The proof is in the same spirit as the proof of the integrability of the characteristic distribution associated to a Poisson structure.

Definition 4.1. Assume that $\mathrm{D} \cap\left(\mathcal{T} \oplus \mathcal{V}_{G}^{\circ}\right)$ and $\mathrm{D} \cap\left(\mathcal{T}_{G} \oplus \mathcal{V}_{G}^{\circ}\right)$ are smooth generalized distributions. The smooth tangent distributions

$$
\mathcal{D}:=\pi_{T M}\left(\mathrm{D} \cap\left(\mathcal{T} \oplus \mathcal{V}_{G}^{\circ}\right)\right) \quad \text { and } \quad \mathcal{D}_{G}:=\pi_{T M}\left(\mathrm{D} \cap\left(\mathcal{T}_{G} \oplus \mathcal{V}_{G}^{\circ}\right)\right)
$$


are called the orbit-type optimal distribution and the isotropy-type optimal distribution.

\subsection{Algebraic involutivity of the optimal distributions}

In this section, we assume that $\mathrm{D} \cap\left(\mathcal{T} \oplus \mathcal{V}_{G}^{\circ}\right)$ is spanned by its descending sections, hence it is smooth. Recall the notation $\mathcal{K}:=\mathcal{V} \oplus\{0\}$. We want to show that, under this assumption, $\mathcal{D}$ and $\mathcal{D}_{G}$ are algebraically involutive. Furthermore, the integrability of $\mathcal{D}_{G}$ will be a consequence of the integrability of $\mathcal{D}$.

Note that in the free case, we have $\mathcal{D}=\mathcal{D}_{G}=\pi_{T M}\left(\mathrm{D} \cap \mathcal{K}^{\perp}\right)$. If $\mathrm{D} \cap \mathcal{K}^{\perp}$ has constant rank on the manifold $M$, it has the structure of a Lie algebroid and, by a standard result in [8], $\mathcal{D}$ is completely integrable. The main result in [19] implies that $D \cap \mathcal{K}^{\perp}$ is then spanned by its descending sections, and hence $\mathcal{D}$ has the same property.

Lemma 4.2. If $(X, \alpha)$ and $(Y, \beta)$ are descending sections of $\mathrm{D}$, then the Courant bracket $[(X, \alpha),(Y, \beta)]$ is also a descending section of $\mathrm{D}$.

Proof. Since D is closed, we have automatically

$$
[(X, \alpha),(Y, \beta)]=\left([X, Y], £_{X} \beta-\mathrm{i}_{Y} \mathrm{~d} \alpha\right) \in \Gamma(\mathrm{D}) .
$$

We have to show that $£_{X} \beta-\mathrm{i}_{Y} \mathrm{~d} \alpha$ is a section of $\mathcal{V}^{\circ}$. Write $X=X^{G}+X^{\mathcal{V}}$ and $Y=$ $Y^{G}+Y^{\mathcal{V}}$ with $X^{G}, Y^{G} \in \mathfrak{X}(M)^{G}$ and $X^{\mathcal{V}}, Y^{\mathcal{V}} \in \Gamma(\mathcal{V})$ (see [18]). For each $\xi \in \mathfrak{g}$, we get

$$
\begin{aligned}
\left(\xi_{X} \beta-\mathrm{i}_{Y} \mathrm{~d} \alpha\right)\left(\xi_{M}\right)= & \left(\mathrm{i}_{X} \mathrm{~d} \beta\right)\left(\xi_{M}\right)+\mathrm{d}\left(\mathrm{i}_{X} \beta\right)\left(\xi_{M}\right)-\left(\mathrm{i}_{Y} \mathrm{~d} \alpha\right)\left(\xi_{M}\right) \\
= & X\left(\beta\left(\xi_{M}\right)\right)-\xi_{M}(\beta(X))-\beta\left(\left[X, \xi_{M}\right]\right)+\xi_{M}(\beta(X)) \\
& -Y\left(\alpha\left(\xi_{M}\right)\right)+\xi_{M}\left(\alpha\left(Y^{G}+Y^{\mathcal{V}}\right)\right)+\alpha\left(\left[Y, \xi_{M}\right]\right) \\
= & X(0)-\xi_{M}(\beta(X))-0+\xi_{M}(\beta(X))-Y(0)+\xi_{M}\left(\alpha\left(Y^{G}\right)\right)+0=0,
\end{aligned}
$$

where we have used the $G$-invariance of the function $\alpha\left(Y^{G}\right),[X, V],[Y, V] \in \mathcal{V}$ for all $V \in \mathcal{V}$, and $\beta(V)=\alpha(V)=0$ for all $V \in \Gamma(\mathcal{V})$. Since the fundamental vector fields $\xi_{M}, \xi \in \mathfrak{g}$, span $\Gamma(\mathcal{V})$ as a $C^{\infty}(M)$-module, we get $£_{X} \beta-\mathrm{i}_{Y} \mathrm{~d} \alpha \in \Gamma\left(\mathcal{V}^{\circ}\right)$.

It remains to show that $£_{X} \beta-\mathrm{i}_{Y} \mathrm{~d} \alpha$ is $G$-equivariant and $[[X, Y], \Gamma(\mathcal{V})] \subseteq \Gamma(\mathcal{V})$. To see this, choose $\xi \in \mathfrak{g}$. Then, using the Jacobi identity for the Courant-Dorfman bracket, 
we get

$$
\begin{aligned}
£_{\xi_{M}}[(X, \alpha),(Y, \beta)] & =\left[\left(\xi_{M}, 0\right),[(X, \alpha),(Y, \beta)]\right]=\left[£_{\xi_{M}}(X, \alpha),(Y, \beta)\right]+\left[(X, \alpha), £_{\xi_{M}}(Y, \beta)\right] \\
& =\left(\left[\left[\xi_{M}, X\right], Y\right]+\left[X,\left[\xi_{M}, Y\right]\right], £_{\left[\xi_{M}, X\right]} \beta-\mathrm{i}_{\left[\xi_{M}, X\right]} \mathrm{d} \alpha\right) \\
& =\left(\left[\left[\xi_{M}, X\right], Y\right]+\left[X,\left[\xi_{M}, Y\right]\right], 0\right) \in \Gamma(\mathcal{V} \oplus 0)
\end{aligned}
$$

since $\left[\xi_{M}, X\right],\left[\xi_{M}, Y\right] \in \Gamma(\mathcal{V})$ and $\beta, \alpha, \mathrm{d} \alpha$ are $G$-equivariant. Hence, since $G$ is a connected Lie group, the 1 -form $£_{X} \beta-\mathrm{i}_{Y} \mathrm{~d} \alpha$ is $G$-invariant.

The following proposition shows the algebraic involutivity of $\mathcal{D}$.

Proposition 4.3. Assume that $\mathrm{D} \cap\left(\mathcal{T} \oplus \mathcal{V}_{G}^{\circ}\right)$ is spanned by its descending sections. Let $(X, \alpha)$ and $(Y, \beta)$ be sections of $\mathrm{D} \cap\left(\mathcal{T} \oplus \mathcal{V}_{G}^{\circ}\right)$. Then the Courant bracket

$$
[(X, \alpha),(Y, \beta)]=\left([X, Y], £_{X} \beta-\mathrm{i}_{Y} \mathrm{~d} \alpha\right)
$$

is also an element of $\Gamma\left(\mathrm{D} \cap\left(\mathcal{T} \oplus \mathcal{V}_{G}^{\circ}\right)\right)$. Hence, if $X, Y \in \Gamma(\mathcal{D})$ then $[X, Y] \in \Gamma(\mathcal{D})$, and $\mathcal{D}$ is algebraically involutive.

Proof. Write $(X, \alpha)$ and $(Y, \beta)$ as sums

$$
(X, \alpha)=\sum_{i=1}^{k} f_{i}\left(X_{i}, \alpha_{i}\right) \quad \text { and } \quad(Y, \beta)=\sum_{j=1}^{l} g_{j}\left(Y_{j}, \beta_{j}\right)
$$

with $f_{1}, \ldots, f_{k}, g_{1}, \ldots, g_{l} \in C^{\infty}(M)$ and $\left(X_{1}, \alpha_{1}\right), \ldots,\left(X_{k}, \alpha_{k}\right),\left(Y_{1}, \beta_{1}\right), \ldots,\left(Y_{l}, \beta_{l}\right)$ descending sections of D. Since

$$
\left[\left(X_{1}, \alpha_{1}\right), f\left(X_{2}, \alpha_{2}\right)\right]=f\left[\left(X_{1}, \alpha_{1}\right),\left(X_{2}, \alpha_{2}\right)\right]+X_{1}(f)\left(X_{2}, \alpha_{2}\right)
$$

for all $\left(X_{1}, \alpha_{1}\right),\left(X_{2}, \alpha_{2}\right) \in \Gamma(\mathrm{D})$ and $f \in C^{\infty}(M)$, we get

$$
\begin{aligned}
{[(X, \alpha),(Y, \beta)] } & =\left[\sum_{i=1}^{k} f_{i}\left(X_{i}, \alpha_{i}\right), \sum_{j=1}^{l} g_{j}\left(Y_{j}, \beta_{j}\right)\right] \\
& =\sum_{i, j=1}^{k, l}\left(g_{j} f_{i}\left[\left(X_{i}, \alpha_{i}\right),\left(Y_{j}, \beta_{j}\right)\right]-g_{j} Y_{j}\left(f_{i}\right)\left(X_{i}, \alpha_{i}\right)+f_{i} X_{i}\left(g_{j}\right)\left(Y_{j}, \beta_{j}\right)\right) .
\end{aligned}
$$


This shows that $[(X, \alpha),(Y, \beta)]$ can be written as a $C^{\infty}(M)$-combination of the descending sections $\left[\left(X_{j}, \alpha_{j}\right),\left(Y_{i}, \beta_{i}\right)\right],\left(X_{j}, \alpha_{j}\right)$, and $\left(Y_{i}, \beta_{i}\right)$ of $\mathrm{D}(i=1, \ldots, k, j=1, \ldots, l)$. The bracket $[(X, \alpha),(Y, \beta)]$ is thus a section of $\mathrm{D} \cap\left(\mathcal{T} \oplus \mathcal{V}_{G}^{\circ}\right)$.

We study now properties of the smooth distribution $\mathcal{D}_{G}$.

Proposition 4.4. If $\mathrm{D} \cap\left(\mathcal{T} \oplus \mathcal{V}_{G}^{\circ}\right)$ is spanned pointwise by its descending sections, then $\mathrm{D} \cap\left(\mathcal{T}_{G} \oplus \mathcal{V}_{G}^{\circ}\right)$ is spanned pointwise by its $G$-invariant descending sections and it is, in particular, smooth.

Proof. Choose $m \in M$, and

$$
\left(v_{m}, l_{m}\right) \in\left(\mathrm{D} \cap\left(\mathcal{T}_{G} \oplus \mathcal{V}_{G}^{\circ}\right)\right)(m) \subseteq\left(\mathrm{D} \cap\left(\mathcal{T} \oplus \mathcal{V}_{G}^{\circ}\right)\right)(m)
$$

Since $\mathrm{D} \cap\left(\mathcal{T} \oplus \mathcal{V}_{G}^{\circ}\right)$ is spanned by its descending sections, we find a smooth section $(X, \alpha)$ of $\mathrm{D} \cap\left(\mathcal{T} \oplus \mathcal{V}_{G}^{\circ}\right)$ such that the vector field $X$ is descending, $\alpha \in \Gamma\left(\mathcal{V}^{\circ}\right)^{G}, X(m)=v_{m}$, and $\alpha(m)=l_{m}$. Since the action of $G$ on $M$ is proper, there exists a tube $U$ for the action at $m$ (see Theorem A.1). Assume that $(X, \alpha)$ is defined on the whole of $U$; otherwise, multiply $(X, \alpha)$ by a bump function that is equal to 1 on a neighborhood $U_{1} \subsetneq U$ of $m$, and equal to 0 on the complement of a neighborhood $U_{2}$ of $m$ with $U_{1} \subsetneq U_{2} \subsetneq U$. Consider the $G$-invariant averaging $\left(X_{G}, \alpha_{G}\right)$ at $m$ of the pair $(X, \alpha)$. Since $(X, \alpha)$ is a section of $\mathrm{D} \cap\left(\mathcal{T} \oplus \mathcal{V}_{G}^{\circ}\right)$, the pair $\left(X_{G}, \alpha_{G}\right)$ is also a section of this intersection, and we get

$$
\begin{aligned}
& X_{G}(m)=X_{G}\left([e, 0]_{H}\right)=\int_{H} T_{[h, 0]_{H}} \Phi_{h^{-1}} X\left([h, 0]_{H}\right) \mathrm{d} h, \\
& \alpha_{G}(m)=\alpha_{G}\left([e, 0]_{H}\right)=\int_{H} \alpha_{[h, 0]_{H}} \circ T_{[e, 0]_{H}} \Phi_{h} \mathrm{~d} h,
\end{aligned}
$$

where $H=G_{m}$ is the isotropy group of the point $m$. Note that $X_{G}(m)$ and $\alpha_{G}(m)$ only depend on the values of $X$ and $\alpha$ at $m$, and thus, the multiplication with the bump function does not change the situation if the section $(X, \alpha)$ was not defined on the whole of $U$. Since $\alpha$ is $G$-invariant and $H$ is the isotropy group of $m$, we have, in particular, $\alpha(m) \circ T_{m} \Phi_{h}=\alpha(m)$, for all $h \in H$ (note that again, the multiplication with the bump function does not change anything). Thus, since $[h, 0]_{H}=\left[h h^{-1}, h \cdot 0\right]_{H}=[e, 0]_{H}=m$, for all $h \in H$, we get

$$
X_{G}(m)=\int_{H} T_{m} \Phi_{h^{-1}} X(m) \mathrm{d} h
$$


and

$$
\alpha_{G}(m)=\int_{H} \alpha(m) \mathrm{d} h=\alpha(m)=l_{m} .
$$

Since $X(m)=v_{m} \in \mathcal{T}_{G}(m)$, it is tangent to the isotropy-type manifold through $m$ and hence, using [10, Lemma 2.4], we conclude that $X_{G}(m)=\int_{H} T_{m} \Phi_{h^{-1}} X(m) \mathrm{d} h=X(m)=v_{m}$. The section $\left(X_{G}, \alpha_{G}\right)$ is hence a $G$-invariant section of $\mathrm{D} \cap\left(\mathcal{T}_{G} \cap \mathcal{V}_{G}^{\circ}\right)$ taking the value $\left(v_{m}, l_{m}\right)$ at $m$.

Theorem 4.5. Assume that $\mathrm{D} \cap\left(\mathcal{T} \oplus \mathcal{V}_{G}^{\circ}\right)$ is spanned pointwise by its descending sections. If $\mathcal{D}$ is an integrable distribution, then $\mathcal{D}_{G}$ is also integrable.

Proof. We have seen in the preceding proposition that $\mathrm{D} \cap\left(\mathcal{T}_{G} \oplus \mathcal{V}_{G}^{\circ}\right)$ is spanned pointwise by its $G$-invariant sections

$$
\left\{(X, \alpha) \in \Gamma(\mathrm{D}) \mid X \in \mathfrak{X}(M)^{G} \text { and } \alpha \in \Gamma\left(\mathcal{V}^{\circ}\right)^{G}\right\} .
$$

Let $(X, \alpha)$ be a descending section of $\mathrm{D}$ and $\phi$ the flow of the vector field $X$. Since $\mathcal{D}$ is integrable, we know that $\phi_{t}^{*} Y$ is a section of $\mathrm{D} \cap\left(\mathcal{T} \oplus \mathcal{V}_{G}^{\circ}\right)$ provided $(Y, \beta)$ is a descending section of $\mathrm{D}$ for all $t$ for which $\phi_{t}$ is defined (see Theorem 2.1 about the integrability of smooth distributions spanned pointwise by families of vector fields).

Consider, in particular, a $G$-invariant pair $(X, \alpha)$ of $\mathrm{D} \cap\left(\mathcal{T}_{G} \oplus \mathcal{V}_{G}^{\circ}\right)$. Then $(X, \alpha)$ is a descending section of $\mathrm{D}$ and, denoting again by $\phi$ the flow of $X$, we know that $\phi_{t}^{*} Y$ is a section of $\mathrm{D} \cap\left(\mathcal{T} \oplus \mathcal{V}_{G}^{\circ}\right)$ if $(Y, \beta)$ is a $G$-invariant descending section of $\mathrm{D}$. But since $X$ and $Y$ are $G$-invariant vector fields, the flow $\phi$ of $X$ is $G$-equivariant and the vector field $\phi_{t}^{*} Y$ is, consequently, $G$-invariant. Let $\beta^{t}$ be a section of $\mathcal{V}_{G}^{\circ}$ such that $\left(\phi_{t}^{*} Y, \beta^{t}\right) \in$ $\Gamma\left(\mathrm{D} \cap\left(\mathcal{T} \oplus \mathcal{V}_{G}^{\circ}\right)\right)$. Since $\phi_{t}^{*} Y \in \mathfrak{X}(M)^{G}$, we even have $\left(\phi_{t}^{*} Y, \beta^{t}\right) \in \Gamma\left(\mathrm{D} \cap\left(\mathcal{T}_{G} \oplus \mathcal{V}_{G}^{\circ}\right)\right)$, and hence $\phi_{t}^{*} Y \in \Gamma\left(\mathcal{D}_{G}\right)$.

By Theorem 2.1, we conclude that $\mathcal{D}_{G}$ is completely integrable in the sense of Stefan and Sussmann.

\subsection{The "exactness" condition}

We make now another assumption: the intersection $D \cap\left(\mathcal{T} \oplus \mathcal{V}_{G}^{\circ}\right)$ is spanned pointwise by its set of exact descending sections

$$
\left\{\left(X_{f}, \mathrm{~d} f\right) \in \Gamma(\mathrm{D}) \mid f \in C^{\infty}(M)^{G},\left[X_{f}, \Gamma(\mathcal{V})\right] \subseteq \Gamma(\mathcal{V})\right\} .
$$

We will show that the (isotropy type) optimal distribution is also integrable in this case. 
Theorem 4.6. If the generalized distribution $D \cap\left(\mathcal{T} \oplus \mathcal{V}_{G}^{\circ}\right)$ is spanned pointwise by its set of exact descending sections, then the distribution $\mathcal{D}_{G}$ is smooth and integrable.

Proof. Since $D \cap\left(\mathcal{T} \oplus \mathcal{V}_{G}^{\circ}\right)$ is spanned pointwise by its exact descending sections, we can show, as in the proof of Proposition 4.4, that $D \cap\left(\mathcal{T}_{G} \oplus \mathcal{V}_{G}^{\circ}\right)$ is spanned pointwise by its exact $G$-invariant descending sections: choose $m \in M$ and $\left(v_{m}, \alpha_{m}\right) \in(\mathrm{D} \cap$ $\left.\left(\mathcal{T}_{G} \oplus \mathcal{V}_{G}^{\circ}\right)\right)(m)$. Then $\left(v_{m}, \alpha_{m}\right) \in\left(\mathrm{D} \cap\left(\mathcal{T} \oplus \mathcal{V}_{G}^{\circ}\right)\right)(m)$ and, by hypothesis, we find a smooth exact descending section $\left(X_{f}, \mathrm{~d} f\right)$ of $\mathrm{D}$ defined in a neighborhood of $m$ and such that $\left(X_{f}, \mathrm{~d} f\right)(m)=\left(v_{m}, \alpha_{m}\right)$. Consider the $G$-invariant average $\left(X_{G}, \alpha_{G}\right)$ of $\left(X_{f}, \mathrm{~d} f\right)$ in a tube centered at $m$. The pair $\left(X_{G}, \alpha_{G}\right)$ is a section of D because D is $G$-invariant. Since $\mathrm{d} f \in \Gamma\left(\mathcal{V}^{\circ}\right)^{G}$, we find $\alpha_{G}=\mathrm{d} f$ and hence $\left(X_{G}, \mathrm{~d} f\right)$ is a $G$-invariant descending section of D. Since $v_{m} \in \mathcal{T}_{G}(m), v_{m}$ is tangent to the isotropy-type manifold through $m$ and we find

$$
X_{G}(m)=\int_{H} T_{m} \Phi_{h^{-1}} X_{f}(m) \mathrm{d} h=\int_{H} T_{m} \Phi_{h^{-1}} v_{m} \mathrm{~d} h=v_{m},
$$

where $H=G_{m}$. Thus, $\left(X_{G}, \mathrm{~d} f\right)$ satisfies $\left(X_{G}, \mathrm{~d} f\right)(m)=\left(v_{m}, \alpha_{m}\right)$.

Hence, the distribution $\mathcal{D}_{G}$ is spanned pointwise by the family

$$
F^{G}:=\left\{X \in \mathfrak{X}(M)^{G} \mid \text { there exists } f \in C^{\infty}(M)^{G} \text { such that }(X, \mathrm{~d} f) \in \Gamma(\mathrm{D})\right\} \subseteq \mathfrak{X}(M) .
$$

We write $X_{f}$ for a $G$-invariant vector field corresponding to the admissible function $f \in C^{\infty}(M)$. Let $X_{f}$ be an element of $F^{G}$ and denote by $\phi$ the flow of $X_{f}$. Let $X_{g}$ be an element of $F^{G}$, corresponding to the admissible function $g \in C^{\infty}(M)^{G}$. By Theorem 2.2, we know that $\left(\phi_{t}^{*} X_{g}, \phi_{t}^{*} \mathrm{~d} g\right)$ is a section of D. Furthermore, since $X_{f}$ is $G$-invariant, its flow $\phi_{t}$ is $G$-equivariant and thus, $\phi_{t}^{*} X_{g} \in \mathfrak{X}(M)^{G}$ and $\phi_{t}^{*} g \in C^{\infty}(M)^{G}$. This shows that $\phi_{t}^{*} X_{g}$ is an element of $F^{G}$ and hence that $\mathcal{D}_{G}$ is completely integrable in the sense of Stefan and Sussmann.

The following proposition is not needed in the rest of the paper; we add it here for the sake of completeness.

Proposition 4.7. If the intersection $D \cap\left(\mathcal{T}_{G} \oplus \mathcal{V}_{G}^{\circ}\right)$ is smooth, then it is spanned pointwise by its $G$-invariant descending sections.

Proof. If $m \in M$ and $\left(v_{m}, l_{m}\right) \in \mathrm{D} \cap\left(\mathcal{T}_{G} \oplus \mathcal{V}_{G}^{\circ}\right)(m)$, then we find a smooth section $(X, \alpha)$ of $\mathrm{D} \cap\left(\mathcal{T}_{G} \oplus \mathcal{V}_{G}^{\circ}\right)$ defined on a neighborhood $U^{\prime}$ of $m$ in $M$ such that $(X(m), \alpha(m))=\left(v_{m}, l_{m}\right)$. 
Let $H=G_{m}$ be the isotropy group of the point $m$ and let $U$ be a tube for the action of $G$ at $m$; assume that $(X, \alpha)$ is defined on the whole of $U$ (otherwise, we multiply $(X, \alpha)$ with a bump function as in the proof of Proposition 4.4). The average $\left(X_{G}, \alpha_{G}\right)$ is a $G$-invariant section of $\mathrm{D} \cap\left(\mathcal{T}_{G} \oplus \mathcal{V}_{G}^{\circ}\right)$, hence a $G$-invariant descending section of $\mathrm{D}$.

As in the proof of Proposition 4.4, we deduce that $X_{G}(m)=X(m)=v_{m}$. Since $\alpha$ is a section of $\mathcal{V}_{G}^{\circ}$, it can be written as $\alpha=\sum_{i=1}^{k} f_{i} \alpha_{i}$, where $f_{i} \in C^{\infty}(M)$ and $\alpha_{i} \in \Gamma\left(\mathcal{V}^{\circ}\right)^{G}$. Again, as in the proof of Proposition 4.4, we compute

$$
\begin{aligned}
\alpha_{G}(m) & =\sum_{i=1}^{k} \int_{H} f_{i}(h m) \alpha_{i}(h m) \circ T_{m} \Phi_{h} \mathrm{~d} h \\
& =\sum_{i=1}^{k} \int_{H} f_{i}(m) \alpha_{i}(m) \mathrm{d} h=\sum_{i=1}^{k} f_{i}(m) \alpha_{i}(m)=\alpha(m)=l_{m} .
\end{aligned}
$$

Hence, we have found a smooth $G$-invariant descending section $\left(X_{G}, \alpha_{G}\right)$ of $\mathrm{D}$ taking the value $\left(v_{m}, l_{m}\right)$ at the point $m$.

Remark 4.8. Let $(M,\{\cdot, \cdot\})$ be a smooth Poisson manifold with a canonical and proper smooth action of a Lie group $G$ on it. Let $D$ be the Dirac structure associated to the Poisson bracket on $M$. Since $\mathcal{V}_{G}^{\circ}$ is generated by the differentials of the $G$-invariant smooth functions, the intersection $D \cap\left(T M \oplus \mathcal{V}_{G}^{\circ}\right)$ is spanned pointwise by the pairs $\left(X_{f}, \mathrm{~d} f\right)$, where $f \in C^{\infty}(M)^{G}$. The vector field $X_{f}$ corresponding to a $G$-invariant function $f \in C^{\infty}(M)^{G}$ is $G$-invariant and we get

$$
\mathrm{D} \cap\left(T M \oplus \mathcal{V}_{G}^{\circ}\right)=\mathrm{D} \cap\left(\mathcal{T} \oplus \mathcal{V}_{G}^{\circ}\right)=\mathrm{D} \cap\left(\mathcal{T}_{G} \oplus \mathcal{V}_{G}^{\circ}\right)
$$

which is spanned by its exact $G$-invariant descending sections. Hence, we are now in the situation of the exactness condition; the distributions $\mathcal{D}_{G}$ and $\mathcal{D}$ are equal and completely integrable in the sense of Stefan and Sussmann.

\section{The Optimal Momentum Maps}

In this section, we define the two optimal momentum maps and show that there is an action of the Lie group on the leaf spaces of the two distributions, such that the momentum maps are $G$-equivariant. We also study the isotropy subgroups of these actions.

Assume that the distributions $\mathcal{D}$ and $\mathcal{D}_{G}$ are spanned pointwise by their descending sections and are completely integrable in the sense of Stefan and Sussmann. Let 
$\mathcal{J}: M \rightarrow M / \mathcal{D}$ and $\mathcal{J}_{G}: M \rightarrow M / \mathcal{D}_{G}$ be the projections on the leaf spaces of $\mathcal{D}$ and $\mathcal{D}_{G}$, respectively. The map $\mathcal{J}$ (respectively, $\mathcal{J}_{G}$ ) is called the (orbit type) Dirac optimal momentum map (respectively, the (isotropy type) Dirac optimal momentum map). In this section, we will study these two momentum maps separately.

\subsection{The orbit-type Dirac optimal momentum map}

We construct an optimal momentum map induced by the distribution $\mathcal{D}$.

Proposition 5.1. If $m$ and $m^{\prime}$ are in the same leaf of $\mathcal{D}$, then $\Phi_{g}(m)$ and $\Phi_{g}\left(m^{\prime}\right)$ are in the same leaf of $\mathcal{D}$ for all $g \in G$. Hence there is a well-defined action $\bar{\Phi}: G \times M / \mathcal{D} \rightarrow M / \mathcal{D}$ given by

$$
\bar{\Phi}_{g}(\mathcal{J}(m)):=\mathcal{J}\left(\Phi_{g}(m)\right)
$$

Proof. Let $m$ and $m^{\prime}$ be in the same leaf of $\mathcal{D}$. Without loss of generality, we can assume that there exists a vector field $X \in \Gamma(\mathcal{D})$ with flow $\phi$ such that $\phi_{t}(m)=m^{\prime}$ for some $t$ (in reality, $m$ and $m^{\prime}$ can be joined by finitely many such curves). Since $(X, \alpha) \in \Gamma(\mathrm{D} \cap(\mathcal{T} \oplus$ $\left.\mathcal{V}_{G}^{\circ}\right)$ ) for some $\alpha \in \Gamma\left(\mathcal{V}_{G}^{\circ}\right)$ and $\mathrm{D} \cap\left(\mathcal{T} \oplus \mathcal{V}_{G}^{\circ}\right)$ is $G$-invariant, it follows that $\left(\Phi_{g}^{*} X, \Phi_{g}^{*} \alpha\right) \in$ $\Gamma\left(\mathrm{D} \cap\left(\mathcal{T} \oplus \mathcal{V}_{G}^{\circ}\right)\right)$ for all $g \in G$. Hence, $\Phi_{g}^{*} X \in \Gamma(\mathcal{D})$, for all $g \in G$. For all $s \in[0, t]$, we have

$$
\frac{\mathrm{d}}{\mathrm{d} s}\left(\Phi_{g} \circ \phi_{s}\right)(m)=T_{\phi_{s}(m)} \Phi_{g}\left(X\left(\phi_{s}(m)\right)\right)=\left(\Phi_{g^{-1}}^{*} X\right)\left(\Phi_{g}\left(\phi_{s}(m)\right)\right) \in \mathcal{D}\left(\left(\Phi_{g} \circ \phi_{s}\right)(m)\right) .
$$

Thus, the curve $c:[0, t] \rightarrow M, \quad s \mapsto\left(\Phi_{g} \circ \phi_{s}\right)(m)$ connecting $c(0)=\Phi_{g}(m)$ to $c(t)=$ $\Phi_{g}\left(\phi_{t}(m)\right)=\Phi_{g}\left(m^{\prime}\right)$ has all its tangent vectors in the distribution $\mathcal{D}$ and hence it lies entirely in the leaf of $\mathcal{D}$ through the point $\Phi_{g}(m)$.

Denote by $G_{(\rho)}$ the isotropy subgroup of $\rho \in M / \mathcal{D}$ for this induced action. If $g \in G_{(\rho)}$ and $m \in \mathcal{J}^{-1}(\rho)$, then

$$
\mathcal{J}\left(\Phi_{g}(m)\right)=\bar{\Phi}_{g}(\mathcal{J}(m))=\bar{\Phi}_{g}(\rho)=\rho=\mathcal{J}(m)
$$

and we conclude, as usual, that $G_{(\rho)}$ leaves $\mathcal{J}^{-1}(\rho)$ invariant. Thus, we get an induced action $\Phi^{(\rho)}: G_{(\rho)} \times \mathcal{J}^{-1}(\rho) \rightarrow \mathcal{J}^{-1}(\rho)$.

Since $\mathcal{D}$ is, by definition, a subdistribution of the integral tangent distribution $\mathcal{T}$, each leaf of $\mathcal{D}$ is be contained in a leaf of $\mathcal{T}$. Since the leaves of $\mathcal{T}$ are the connected 
components of the orbit-type submanifolds of $M$, the induced action $\Phi^{(\rho)}$ of $G_{(\rho)}$ on $\mathcal{J}^{-1}(\rho)$ has isotropy subgroups that are conjugated in $G$ for each $\rho \in M / \mathcal{D}_{G}$, as is shown in the next proposition.

Proposition 5.2. Let $\mathcal{J}^{-1}(\rho)$ be a leaf of $\mathcal{D}$ and $m \in \mathcal{J}^{-1}(\rho)$. Then the isotropy subgroup $\left(G_{(\rho)}\right)_{m}$ of $m$ by the action of $G_{(\rho)}$ on $\mathcal{J}^{-1}(\rho)$ is equal to the isotropy subgroup $G_{m}$. Hence we have the inclusion

$$
\bigcup_{\mathcal{J}(m)=\rho} G_{m} \subseteq G_{(\rho)} .
$$

Proof. Choose $m \in \mathcal{J}^{-1}(\rho)$ and $g \in\left(G_{(\rho)}\right)_{m}$. Then we have $g \cdot m=m$, which leads to $g \in G_{m}$. Conversely, choose $g \in G_{m}$ and compute

$$
\bar{\Phi}_{g}(\rho)=\bar{\Phi}_{g}(\mathcal{J}(m))=\mathcal{J}(g \cdot m)=\mathcal{J}(m)=\rho .
$$

Thus, we have shown that $g \in G_{(\rho)}$. But since $g \cdot m=m$, we have in particular $g \in\left(G_{(\rho)}\right)_{m}$.

We will see later that the isotropy subgroups of the action of $G_{(\rho)}$ on $\mathcal{J}^{-1}(\rho)$ are conjugated in $G_{(\rho)}$.

In general, the action of $G_{(\rho)}$ on $\mathcal{J}^{-1}(\rho)$ is not proper. A sufficient condition for this is, for example, the closedness of $G_{(\rho)}$ in $G$, which is not true, in general.

\subsection{The isotropy-type Dirac optimal momentum map}

The optimal distribution $\mathcal{D}_{G}$ gives rise to a second Dirac optimal momentum map. The results are analogous to those in the previous subsection.

Proposition 5.3. If $m$ and $m^{\prime}$ are in the same leaf of $\mathcal{D}_{G}$, then $\Phi_{g}(m)$ and $\Phi_{g}\left(m^{\prime}\right)$ are in the same leaf of $\mathcal{D}_{G}$ for all $g \in G$. Hence there is a well-defined action $\bar{\Phi}: G \times M / \mathcal{D}_{G} \rightarrow$ $M / \mathcal{D}_{G}$ given by

$$
\bar{\Phi}_{g}\left(\mathcal{J}_{G}(m)\right):=\mathcal{J}_{G}\left(\Phi_{g}(m)\right)
$$

Proof. The proof is almost identical to that of Proposition 5.1. The only difference is that we have to consider the flow of $X \in \Gamma\left(\mathcal{D}_{G}\right)$. Then we use $G$-invariance of the distribution $\mathrm{D} \cap\left(\mathcal{T}_{G} \oplus \mathcal{V}_{G}^{\circ}\right)$. 
Let $G_{\sigma}$ be the isotropy subgroup of $\sigma \in M / \mathcal{D}_{G}$. Then there exists a unique smooth structure on $G_{\sigma}$ with respect to which $G_{\sigma}$ is an initial Lie subgroup of $G$; the Lie algebra of $G_{\sigma}$ is

$$
\mathfrak{g}_{\sigma}:=\left\{\xi \in \mathfrak{g} \mid \xi_{M}(m) \in \mathcal{D}_{G}(m) \text { for all } m \in \mathcal{J}_{G}^{-1}(\sigma)\right\}
$$

(see [23, Proposition 3.4.4]). In particular,

$$
\operatorname{dim} \mathfrak{g}_{\sigma}=\operatorname{dim}\left(\mathcal{V}(m) \cap \mathcal{D}_{G}(m)\right)+\operatorname{dim} G_{m} \quad \text { for any } m \in \mathcal{J}_{G}^{-1}(\sigma)
$$

Indeed, the surjective linear map $\xi \in \mathfrak{g}_{\sigma} \mapsto \xi_{M}(m) \in \mathcal{V}(m) \cap \mathcal{D}_{G}(m)$ has kernel $\{\xi \in \mathfrak{g} \mid$ $\left.\xi_{M}(m)=0\right\}$.

Since $G_{\sigma}$ leaves $\mathcal{J}_{G}^{-1}(\sigma)$ invariant, we get an induced action $\Phi^{\sigma}$ of $G_{\sigma}$ on $\mathcal{J}_{G}^{-1}(\sigma)$. Since $\mathcal{D}_{G} \subseteq \mathcal{T}_{G}$, the points of $\mathcal{J}_{G}^{-1}(\sigma)$ are all in the same connected component of an isotropy type submanifold of $M$ and hence $G_{m}=H_{\sigma}$ for all $m \in \mathcal{J}_{G}^{-1}(\sigma)$ and some compact subgroup $H_{\sigma} \subseteq G$.

Proposition 5.4. Let $\mathcal{J}_{G}^{-1}(\sigma)$ be a leaf of $\mathcal{D}_{G}$ and $m \in \mathcal{J}_{G}^{-1}(\sigma)$. The isotropy subgroup $\left(G_{\sigma}\right)_{m}$ of $m$ by the action of $G_{\sigma}$ on $\mathcal{J}_{G}^{-1}(\sigma)$ is equal to the compact subgroup $H_{\sigma}$ which is automatically a subset of $G_{\sigma}$.

Proof. Here also, the proof of the analog in the orbit-type case (Proposition 5.2) can be adapted to this particular situation.

With this last proposition, we can show the missing detail in the preceding subsection.

Proposition 5.5. Choose $\rho \in M / \mathcal{D}$ and let $G_{(\rho)}$ be its isotropy subgroup by the action of $G$ on $M / \mathcal{D}$. Then we find for all $m, m^{\prime} \in \mathcal{J}^{-1}(\rho)$ an element $g \in G_{(\rho)}$ such that $G_{m}=$ $g G_{m^{\prime}} g^{-1}$.

Proof. We have $\mathcal{D}_{G} \subseteq \mathcal{D} \subseteq \mathcal{V}+\mathcal{D}_{G}$, by definition. For $m \in M$, the leaf $\mathcal{J}^{-1}(\rho)$ of $\mathcal{D}$ through $m$ is thus contained in $G \cdot \mathcal{J}_{G}^{-1}(\sigma)$, where $\sigma=\mathcal{J}_{G}(m)$ and $\rho=\mathcal{J}(m)$, and the leaf $\mathcal{J}_{G}^{-1}(\sigma)$ is contained in $\mathcal{J}^{-1}(\rho)$.

Choose $m^{\prime} \in \mathcal{J}^{-1}(\rho)$. Then $m^{\prime}$ can be written as $m^{\prime}=g m^{\prime \prime}$ with $m^{\prime \prime} \in \mathcal{J}_{G}^{-1}(\sigma) \subseteq$ $\mathcal{J}^{-1}(\rho)$ and $g \in G$. Then, we have $G_{m^{\prime}}=g G_{m^{\prime \prime}} g^{-1}$. But since $m^{\prime \prime}$ is an element of 
$\mathcal{J}_{G}^{-1}\left(\mathcal{J}_{G}(m)\right) \subseteq M_{G_{m}}$, we have $G_{m^{\prime \prime}}=G_{m}$ and hence $G_{m^{\prime}}=g G_{m} g^{-1}$. Furthermore, $g \cdot \rho=$ $g \cdot \mathcal{J}(m)=g \cdot \mathcal{J}\left(m^{\prime \prime}\right)=\mathcal{J}\left(g \cdot m^{\prime \prime}\right)=\mathcal{J}\left(m^{\prime}\right)=\rho$, which shows that $g \in G_{(\rho)}$.

\subsection{Universality of the optimal map $\mathcal{J}_{G}$ under the exactness condition (§ 4.2)}

We assume here that the intersection $\mathrm{D} \cap\left(\mathcal{T} \oplus \mathcal{V}_{G}^{\circ}\right)$ is spanned pointwise by its set of exact descending sections

$$
\left\{\left(X_{f}, \mathrm{~d} f\right) \in \Gamma(\mathrm{D}) \mid f \in C^{\infty}(M)^{G},\left[X_{f}, \Gamma(\mathcal{V})\right] \subseteq \Gamma(\mathcal{V})\right\}
$$

We have shown in Section 4.2 that the distribution $\mathcal{D}_{G}$ is then completely integrable in the sense of Stefan and Sussmann. Hence, the isotropy-type optimal momentum map is defined.

Note that in this particular case, the smooth distribution $\mathcal{D}_{G}$ is spanned pointwise by the following family of vector fields:

$$
\mathcal{F}=\left\{X_{f} \in \mathfrak{X}(M)^{G} \mid\left(X_{f}, \mathrm{~d} f\right) \in \Gamma(\mathrm{D}), f \in C^{\infty}(M)^{G}\right\},
$$

and its leaves are hence the accessible sets of this family of vector fields. Using this, we prove a universality property of the isotropy-type optimal momentum map. This theorem suggests that the isotropy-type optimal momentum map should be the more "natural" one, provided that the exactness condition above is satisfied. Recall that in the Poisson case, the optimal distribution is always spanned by the family $\mathcal{F}$, and the following statement is hence true (see [23]).

Theorem 5.6. Let $G$ be a symmetry Lie group of the Dirac manifold ( $M, \mathrm{D})$ and $\mathrm{J}: M \rightarrow P$ a function with the Noether property (see Definition 3.5). Then there exists a unique map $\phi_{G}: M / \mathcal{D}_{G} \rightarrow P$ such that the following diagram commutes:

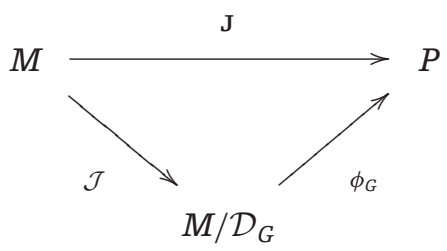


If $\mathbf{J}$ is $G$-equivariant with respect to some $G$-action on $P$, then $\phi_{G}$ is also $G$-equivariant. If $\mathbf{J}$ is smooth and $M / \mathcal{D}_{G}$ is a smooth manifold, then $\phi_{G}$ is also smooth.

Proof. The proof is the same as for Poisson manifolds (see [23, Theorem 5.5.15]). Define $\phi: M / \mathcal{D}_{G} \rightarrow P$ by $\phi(\rho):=\mathbf{J}(m)$, where $\rho=\mathcal{J}(m)$. The map $\phi$ is well defined since if $m^{\prime} \in$ $\mathcal{J}^{-1}(\rho)$, then there is a finite composition $F$ of flows of elements of $\mathcal{F}$ such that $m^{\prime}=$ $F(m)$. Since $\mathbf{J}$ is a Noether momentum map, we have

$$
\mathbf{J}\left(m^{\prime}\right)=\mathbf{J}(F(m))=\mathbf{J}(m)=\phi(\rho) .
$$

The definition immediately implies that the diagram commutes. Uniqueness of $\phi$ follows from the requirement that the diagram commutes and the surjectivity of $\mathcal{J}$. Equivariance of $\phi$ is a direct consequence of the definition (14) of the $G$-action on $M / \mathcal{D}_{G}$. Finally, if all objects are smooth manifolds and $\mathcal{J}, \mathbf{J}$ are smooth maps, then $\phi$ is a smooth map as the quotient of the smooth map $\mathbf{J}$ by the projection $\mathcal{J}$ (see [5]).

\section{Optimal Reduction}

In this section, we generalize the optimal reduction theorem for Poisson manifolds (see [23, Theorem 9.1.1]) to closed Dirac manifolds. As we shall see, with necessary assumptions and appropriately extended definitions, this important desingularization method works also for Dirac manifolds.

We shall assume throughout this section that the distributions $\mathcal{D}$ (respectively, $\mathcal{D}_{G}$ ) are spanned pointwise by their descending (respectively, $G$-invariant) sections and are completely integrable in the sense of Stefan and Sussmann. Recall from Section 2.1.2 that their leaves are then the accessible sets of these special families of vector fields.

As we have seen above, there are two optimal momentum maps that we can consider. We have hence two optimal point reduction theorems. We will also prove an orbit reduction theorem and shall see that the two optimal momentum maps give rise to the same orbit reduction theorem. At the end of this section, it will be shown that the three optimally reduced manifolds at $\mathcal{J}(m)$ and $\mathcal{J}_{G}(m)$, for a point $m \in M$, are isomorphic presymplectic manifolds.

\subsection{The reduction theorems: Optimal point reduction by Dirac actions}

In this subsection, ( $M, \mathrm{D})$ is again a smooth integrable Dirac manifold and $G$ is a Lie group acting smoothly and properly on $M$ and leaving the Dirac structure invariant. We 
assume that $\mathrm{D} \cap\left(\mathcal{T} \oplus \mathcal{V}_{G}^{\circ}\right)$ is spanned pointwise by the descending sections of $\mathrm{D}$ and that $\mathcal{D}$ is integrable. Let $\mathcal{J}: M \rightarrow M / \mathcal{D}$ be the orbit-type Dirac optimal momentum map associated to this action. If $\rho \in M / \mathcal{D}$, we denote by $\iota_{(\rho)}: \mathcal{J}^{-1}(\rho) \hookrightarrow M$ the regular immersion. Recall that $\mathcal{J}^{-1}(\rho)$ is an initial submanifold of $M$.

Theorem 6.1. For any $\rho \in M / \mathcal{D}$ with isotropy subgroup $G_{(\rho)}$ acting properly on $\mathcal{J}^{-1}(\rho)$, the orbit space $M_{(\rho)}:=\mathcal{J}^{-1}(\rho) / G_{(\rho)}$ is a regular quotient manifold such that the projection $\pi_{(\rho)}: \mathcal{J}^{-1}(\rho) \rightarrow M_{(\rho)}$ is a smooth submersion. Define $\omega_{(\rho)} \in \Omega^{2}\left(M_{(\rho)}\right)$ by

$$
\left(\pi_{(\rho)}^{*} \omega_{(\rho)}\right)(m)\left(v_{m}, w_{m}\right):=\alpha_{\left.\iota_{l \rho}\right)}(m)\left(Y\left(\iota_{(\rho)}(m)\right)\right)=-\beta_{\iota_{(\rho)}(m)}\left(X\left(\iota_{(\rho)}(m)\right)\right),
$$

for any $m \in \mathcal{J}^{-1}(\rho)$ and any $X, Y \in \Gamma(\mathcal{D})$ defined on an open set around $\iota_{(\rho)}(m)$, where $(X, \alpha),(Y, \beta) \in \Gamma\left(\mathrm{D} \cap\left(\mathcal{T} \oplus \mathcal{V}_{G}^{\circ}\right)\right)$ are such that $T_{m} \iota_{(\rho)} v_{m}=X\left(\iota_{(\rho)}(m)\right)$ and $T_{m} \iota_{(\rho)} w_{m}=$ $Y\left(\iota_{(\rho)}(m)\right)$.

Then $\left(M_{(\rho)}, \omega_{(\rho)}\right)$ is a presymplectic manifold. The pair $\left(M_{(\rho)}, \mathrm{D}_{(\rho)}\right)$ is called the orbit-type Dirac optimal point reduced space of $(M, \mathrm{D})$ at $\rho$, where $\mathrm{D}_{(\rho)}$ is the graph of the presymplectic form $\omega_{(\rho)}$.

Since $\mathrm{D} \cap\left(\mathcal{T} \oplus \mathcal{V}_{G}^{\circ}\right)$ is spanned pointwise by its descending sections and $\mathcal{D}$ is integrable, $\mathcal{D}_{G}=\pi_{T M}\left(\mathrm{D} \cap\left(\mathcal{T}_{G} \oplus \mathcal{V}_{G}^{\circ}\right)\right)$ is spanned pointwise by its $G$-invariant descending sections and is completely integrable in the sense of Stefan and Sussmann by Proposition 4.4 and Theorem 4.5. Let $\mathcal{J}_{G}: M \rightarrow M / \mathcal{D}_{G}$ be the isotropy-type Dirac optimal momentum map. For $\sigma \in M / \mathcal{D}_{G}$, denote by $\iota_{\sigma}: \mathcal{J}_{G}^{-1}(\sigma) \hookrightarrow M$ the regular immersion.

Theorem 6.2. For any $\sigma \in M / \mathcal{D}_{G}$ with isotropy subgroup $G_{\sigma}$ acting properly on $\mathcal{J}_{G}^{-1}(\sigma)$, the orbit space $M_{\sigma}:=\mathcal{J}_{G}^{-1}(\sigma) / G_{\sigma}$ is a regular quotient manifold such that the projection $\pi_{\sigma}: \mathcal{J}^{-1}(\sigma) \rightarrow M_{\sigma}$ is a smooth submersion. Define $\omega_{\sigma} \in \Omega^{2}\left(M_{\sigma}\right)$ by

$$
\left(\pi_{\sigma}^{*} \omega_{\sigma}\right)(m)\left(v_{m}, w_{m}\right):=\alpha_{l_{\sigma}(m)}\left(Y\left(\iota_{\sigma}(m)\right)\right)=-\beta_{\iota_{\sigma}(m)}\left(X\left(\iota_{\sigma}(m)\right)\right),
$$

for any $m \in \mathcal{J}^{-1}(\sigma)$ and any $X, Y \in \Gamma\left(\mathcal{D}_{G}\right)$ defined on an open set around $\iota_{\sigma}(m)$, where $(X, \alpha),(Y, \beta) \in \Gamma\left(\mathrm{D} \cap\left(\mathcal{T}_{G} \oplus \mathcal{V}_{G}^{\circ}\right)\right)$ are such that $T_{m} \iota_{\sigma} v_{m}=X\left(\iota_{\sigma}(m)\right)$ and $T_{m} \iota_{\sigma} w_{m}=Y\left(\iota_{\sigma}(m)\right)$.

Then $\left(M_{\sigma}, \omega_{\sigma}\right)$ is a presymplectic manifold. The pair $\left(M_{\sigma}, \mathrm{D}_{\sigma}\right)$ is called the isotropy-type Dirac optimal point reduced space of $(M, \mathrm{D})$ at $\sigma$, where $\mathrm{D}_{\sigma}$ is the graph of the presymplectic form $\omega_{\sigma}$. 
Proof of Theorem 6.1. Recall the notation $\Phi^{(\rho)}: G_{(\rho)} \times \mathcal{J}^{-1}(\rho) \rightarrow \mathcal{J}^{-1}(\rho)$ for the restriction of the original $G$-action on $M$ to the Lie group $G_{(\rho)}$ and the manifold $\mathcal{J}^{-1}(\rho)$. Since the $G_{(\rho)}$-action on $\mathcal{J}^{-1}(\rho)$ is, by hypothesis, proper and its isotropy subgroups are conjugated by Proposition 5.5, the quotient $M_{(\rho)}:=\mathcal{J}^{-1}(\rho) / G_{(\rho)}$ is a regular quotient manifold and the projection $\pi_{(\rho)}: \mathcal{J}^{-1}(\rho) \rightarrow \mathcal{J}^{-1}(\rho) / G_{(\rho)}$ is a smooth surjective submersion.

We show that $\omega_{(\rho)}$ given by (16) is well defined. Let $m, m^{\prime} \in \mathcal{J}^{-1}(\rho)$ be such that $\pi_{(\rho)}(m)=\pi_{(\rho)}\left(m^{\prime}\right)$ and let $v, w \in T_{m} \mathcal{J}^{-1}(\rho), v^{\prime}, w^{\prime} \in T_{m^{\prime}} \mathcal{J}^{-1}(\rho)$ be such that $T_{m} \pi_{(\rho)}(v)=$ $T_{m^{\prime} \pi_{(\rho)}}\left(v^{\prime}\right), T_{m} \pi_{(\rho)}(w)=T_{m^{\prime} \pi_{(\rho)}}\left(w^{\prime}\right)$. Let $(X, \alpha),\left(X^{\prime}, \alpha^{\prime}\right),(Y, \beta),\left(Y^{\prime}, \beta^{\prime}\right)$ be sections of $\mathrm{D} \cap(\mathcal{T} \oplus$ $\left.\mathcal{V}_{G}^{\circ}\right)$ such that

$$
\begin{aligned}
& X\left(\iota_{(\rho)} m\right)=T_{m} \iota_{(\rho)} v, \quad X^{\prime}\left(\iota_{(\rho)}\left(m^{\prime}\right)\right)=T_{m^{\prime} \iota_{(\rho)}} v^{\prime}, \\
& Y\left(\iota_{(\rho)}(m)\right)=T_{m} \iota_{(\rho)} w, \quad Y^{\prime}\left(\iota_{(\rho)}\left(m^{\prime}\right)\right)=T_{m^{\prime} \iota_{(\rho)}} w^{\prime} .
\end{aligned}
$$

The condition $\pi_{(\rho)}(m)=\pi_{(\rho)}\left(m^{\prime}\right)$ implies the existence of an element $g \in G_{(\rho)} \subseteq G$ such that $m^{\prime}=\Phi_{g}^{(\rho)}(m)$. We have then $\pi_{(\rho)}=\pi_{(\rho)} \circ \Phi_{g}^{(\rho)}$ and thus $T_{m} \pi_{(\rho)}=T_{m^{\prime}} \pi_{(\rho)} \circ T_{m} \Phi_{g}^{(\rho)}$. Furthermore, because of the equalities $T_{m} \pi_{(\rho)}(v)=T_{m^{\prime}} \pi_{(\rho)}\left(v^{\prime}\right), T_{m} \pi_{(\rho)}(w)=T_{m^{\prime}} \pi_{(\rho)}\left(w^{\prime}\right)$, we have

$$
T_{m^{\prime}} \pi_{(\rho)}\left(T_{m} \Phi_{g}^{(\rho)}(v)-v^{\prime}\right)=0 \quad \text { and } \quad T_{m^{\prime}} \pi_{(\rho)}\left(T_{m} \Phi_{g}^{(\rho)}(w)-w^{\prime}\right)=0
$$

and there exist elements $\xi^{1}, \xi^{2} \in \mathfrak{g}$ such that $\xi_{M}^{1}\left(\iota_{(\rho)}\left(m^{\prime}\right)\right), \xi_{M}^{2}\left(\iota_{(\rho)}\left(m^{\prime}\right)\right) \in \mathcal{D}\left(\iota_{(\rho)}\left(m^{\prime}\right)\right)$,

$$
\begin{aligned}
X^{\prime}\left(\iota_{(\rho)}\left(m^{\prime}\right)\right)-T_{l_{(\rho)}(m)} \Phi_{g}\left(X\left(\iota_{(\rho)}(m)\right)\right) & =T_{\iota_{(\rho)}\left(m^{\prime}\right)}\left(v^{\prime}-T_{m} \Phi_{g}^{(\rho)}(v)\right)=T_{l_{(\rho)}\left(m^{\prime}\right)} \xi_{\mathcal{J}^{-1}(\rho)}^{1}\left(m^{\prime}\right) \\
& =\xi_{M}^{1}\left(\iota_{(\rho)}\left(m^{\prime}\right)\right)
\end{aligned}
$$

and

$$
\begin{aligned}
Y^{\prime}\left(\iota_{(\rho)}\left(m^{\prime}\right)\right)-T_{l_{(\rho)}(m)} \Phi_{g}\left(Y\left(\iota_{(\rho)}(m)\right)\right) & =T_{l_{(\rho)}\left(m^{\prime}\right)}\left(w^{\prime}-T_{m} \Phi_{g}^{(\rho)}(w)\right)=T_{l_{(\rho)}\left(m^{\prime}\right)} \xi_{\mathcal{J}^{-1}(\rho)}^{2}\left(m^{\prime}\right) \\
& =\xi_{M}^{2}\left(\iota_{(\rho)}\left(m^{\prime}\right)\right),
\end{aligned}
$$

where we have used the equality $\iota_{(\rho)} \circ \Phi_{g}^{(\rho)}=\Phi_{g} \circ \iota_{(\rho)}$. This yields

$$
X^{\prime}\left(n^{\prime}\right)=\left(\left(\Phi_{g^{-1}}\right)^{*} X\right)\left(n^{\prime}\right)+\xi_{M}^{1}\left(n^{\prime}\right) \quad \text { and } \quad Y^{\prime}\left(n^{\prime}\right)=\left(\left(\Phi_{g^{-1}}\right)^{*} Y\right)\left(n^{\prime}\right)+\xi_{M}^{2}\left(n^{\prime}\right),
$$


where we let $n:=\iota_{(\rho)}(m)$ and $n^{\prime}:=\iota_{(\rho)}\left(m^{\prime}\right)$. Since $\left(X^{\prime}, \alpha^{\prime}\right)$ and $\left(\left(\Phi_{g^{-1}}\right)^{*} Y,\left(\Phi_{g^{-1}}\right)^{*} \beta\right)$ are sections of $\mathrm{D}$ in a neighborhood of the point $n^{\prime}$, we have

$$
\left(\left(\Phi_{g^{-1}}\right)^{*} \beta\right)\left(X^{\prime}\right)=-\alpha^{\prime}\left(\left(\Phi_{g^{-1}}\right)^{*} Y\right)
$$

and thus we conclude

$$
\begin{aligned}
& \omega_{(\rho)}\left(\pi_{(\rho)}\left(m^{\prime}\right)\right)\left(T_{m^{\prime}} \pi_{(\rho)}\left(v^{\prime}\right), T_{m^{\prime}} \pi_{(\rho)}\left(w^{\prime}\right)\right)=\left(\pi_{(\rho)}^{*} \omega_{(\rho)}\right)\left(m^{\prime}\right)\left(v^{\prime}, w^{\prime}\right) \\
& =\alpha^{\prime}\left(n^{\prime}\right)\left(Y^{\prime}\left(n^{\prime}\right)\right)=\alpha^{\prime}\left(n^{\prime}\right)\left(\left(\left(\Phi_{g^{-1}}\right)^{*} Y\right)\left(n^{\prime}\right)+\xi_{M}^{2}\left(n^{\prime}\right)\right) \\
& =\alpha^{\prime}\left(n^{\prime}\right)\left(\left(\left(\Phi_{g^{-1}}\right)^{*} Y\right)\left(n^{\prime}\right)\right)+\alpha^{\prime}\left(n^{\prime}\right)\left(\xi_{M}^{2}\left(n^{\prime}\right)\right) \\
& \stackrel{(*)}{=} \alpha^{\prime}\left(n^{\prime}\right)\left(\left(\left(\Phi_{g^{-1}}\right)^{*} Y\right)\left(n^{\prime}\right)\right) \stackrel{(18)}{=}-\left(\left(\Phi_{g^{-1}}\right)^{*} \beta\right)\left(n^{\prime}\right)\left(X^{\prime}\left(n^{\prime}\right)\right) \\
& =-\left(\left(\Phi_{g^{-1}}\right)^{*} \beta\right)\left(n^{\prime}\right)\left(\left(\left(\Phi_{g^{-1}}\right)^{*} X\right)\left(n^{\prime}\right)+\xi_{M}^{1}\left(n^{\prime}\right)\right) \\
& \stackrel{(*)}{=}-\left(\left(\Phi_{g^{-1}}\right)^{*} \beta\right)\left(n^{\prime}\right)\left(\left(\left(\Phi_{g^{-1}}\right)^{*} X\right)\left(n^{\prime}\right)\right)=-\beta(n)(X(n)) \\
& =\omega_{(\rho)}\left(\pi_{(\rho)}(m)\right)\left(T_{m} \pi_{(\rho)}(v), T_{m} \pi_{(\rho)}(w)\right) .
\end{aligned}
$$

For the equalities $(*)$, we use the fact that $\alpha^{\prime}$ and $\Phi_{g^{-1}}^{*} \beta$ are sections of $\mathcal{V}^{\circ}$.

Finally, we show that $\omega_{(\rho)}$ is closed. Let $m \in \mathcal{J}^{-1}(\rho)$ and choose $\tilde{X}, \tilde{Y}, \tilde{Z} \in$ $\mathfrak{X}\left(\mathcal{J}^{-1}(\rho)\right)$, defined on a neighborhood of $m$ in $\mathcal{J}^{-1}(\rho)$. Then there exist sections $(X, \alpha)$, $(Y, \beta),(Z, \gamma) \in \Gamma\left(\mathrm{D} \cap\left(\mathcal{T} \oplus \mathcal{V}_{G}^{\circ}\right)\right)$ defined on a neighborhood of $\iota_{(\rho)}(m)$ in $M$ such that

$$
\tilde{X} \sim_{l_{(\rho)}} X, \quad \tilde{Y} \sim_{\iota_{(\rho)}} Y, \quad \text { and } \quad \tilde{Z} \sim_{\iota_{(\rho)}} Z
$$

Since $[\tilde{X}, \tilde{Y}] \sim_{\iota_{(\rho)}}[X, Y]$ and

$$
\left([X, Y], £_{X} \beta-\mathrm{i}_{Y} \mathrm{~d} \alpha\right) \in \Gamma(\mathrm{D}),
$$

we have (by definition (16))

$$
\left(\pi_{(\rho)}^{*} \omega_{(\rho)}\right)([\tilde{X}, \tilde{Y}], \tilde{Z})=-\gamma([X, Y]) \circ \iota_{(\rho)}=\left(£_{X} \beta-\mathrm{i}_{Y} \mathrm{~d} \alpha\right)(Z) \circ \iota_{(\rho)} .
$$

Thus, recalling the definition (16), we get

$$
\begin{aligned}
\mathbf{d}\left(\pi_{(\rho)}^{*} \omega_{(\rho)}\right)(\tilde{X}, \tilde{Y}, \tilde{Z})= & \tilde{X}\left[\left(\pi_{(\rho)}^{*} \omega_{(\rho)}\right)(\tilde{Y}, \tilde{Z})\right]-\tilde{Y}\left[\left(\pi_{(\rho)}^{*} \omega_{(\rho)}\right)(\tilde{X}, \tilde{Z})\right] \\
& +\tilde{Z}\left[\left(\pi_{(\rho)}^{*} \omega_{(\rho)}\right)(\tilde{X}, \tilde{Y})\right]-\left(\pi_{(\rho)}^{*} \omega_{(\rho)}\right)([\tilde{X}, \tilde{Y}], \tilde{Z})
\end{aligned}
$$




$$
\begin{aligned}
& +\left(\pi_{(\rho)}^{*} \omega_{(\rho)}\right)([\tilde{X}, \tilde{Z}], \tilde{Y})-\left(\pi_{(\rho)}^{*} \omega_{(\rho)}\right)([\tilde{Y}, \tilde{Z}], \tilde{X}) \\
\stackrel{(19)}{=} & \tilde{X}\left[\beta(Z) \circ \iota_{(\rho)}\right]+\tilde{Y}\left[\gamma(X) \circ \iota_{(\rho)}\right]+\tilde{Z}\left[\alpha(Y) \circ \iota_{(\rho)}\right] \\
& +\gamma([X, Y]) \circ \iota_{(\rho)}+\left(E_{X} \gamma-\mathrm{i}_{Z} \mathrm{~d} \alpha\right)(Y) \circ \iota_{(\rho)}+\alpha([Y, Z]) \circ \iota_{(\rho)} \\
= & (X[\beta(Z)]+Y[\gamma(X)]+Z[\alpha(Y)]+\gamma([X, Y])-\gamma([X, Y]) \\
& \left.+X[\gamma(Y)]-Z[\alpha(Y)]+Y[\alpha(Z)]+\alpha([Z, Y])+\alpha([Y, Z])) \circ \iota_{(\rho)}\right) \\
= & (X[\beta(Z)+\gamma(Y)]+Y[\gamma(X)+\alpha(Z)]) \circ \iota_{(\rho)}=0,
\end{aligned}
$$

where we used the fact that $\gamma(X)+\alpha(Z)=0$ and $\gamma(Y)+\beta(Z)=0$ (this follows directly from $(X, \alpha),(Y, \beta),(Z, \gamma) \in \Gamma(\mathrm{D}))$. Thus, $\pi_{(\rho)}^{*} \mathbf{d} \omega_{(\rho)}=\mathbf{d}\left(\pi_{(\rho)}^{*} \omega_{(\rho)}\right)=0$ and, because $\pi_{(\rho)}$ is a surjective submersion, this yields $\mathbf{d} \omega_{(\rho)}=0$. Therefore, $\omega_{(\rho)}$ is a well-defined presymplectic form on $M_{(\rho)}$.

Theorem 6.2 has a similar proof.

\subsection{Optimal orbit reduction}

Let $(M, \mathrm{D})$ be a smooth integrable Dirac manifold with a smooth and proper canonical action of a Lie group $G$ on it. Assume that the same conditions on $D \cap\left(\mathcal{T} \oplus \mathcal{V}_{G}^{\circ}\right)$ as in the preceding subsection are satisfied, that is, $\mathrm{D} \cap\left(\mathcal{T} \oplus \mathcal{V}_{G}^{\circ}\right)$ is spanned pointwise by the descending sections of $D$ and $\mathcal{D}$ is integrable.

Let $\mathcal{J}: M \rightarrow M / \mathcal{D}, \mathcal{J}_{G}: M \rightarrow M / \mathcal{D}_{G}$ be the optimal momentum maps. Consider the distribution $\mathcal{D}_{G}+\mathcal{V} \subseteq T M$. By Ortega and Ratiu [23, Proposition 3.4.6], its integrability follows from the integrability of $\mathcal{D}$ and Theorem 4.5. Since $G$ is connected, the leaves of $\mathcal{D}_{G}+\mathcal{V}$ are the sets $G \cdot \mathcal{J}_{G}^{-1}(\sigma)=G \cdot \mathcal{J}^{-1}\left(\mathcal{J}(m)\right.$ ) for any $m \in \mathcal{J}_{G}^{-1}(\sigma)$ (recall that $\mathcal{J}^{-1}(\mathcal{J}(m)) \subseteq G \cdot \mathcal{J}_{G}^{-1}(\sigma)$ for any $\left.m \in \mathcal{J}_{G}^{-1}(\sigma)\right)$. The leaves of $\mathcal{D}_{G}+\mathcal{V}$ are initial submanifolds of $M$; that is, the maps $\iota_{\sigma, G}: G \cdot \mathcal{J}_{G}^{-1}(\sigma) \hookrightarrow M$ are regular immersions for all $\sigma \in M / \mathcal{D}_{G}$.

Lemma 6.3. Choose $\sigma \in M / \mathcal{D}_{G}$ such that the action of $G_{\sigma}$ on $\mathcal{J}_{G}^{-1}(\sigma)$ is proper. The integral leaf $G \cdot \mathcal{J}_{G}^{-1}(\sigma)$ of $\mathcal{D}_{G}+\mathcal{V}$ is diffeomorphic to the regular quotient manifold

$$
G \times{ }_{G_{\sigma}} \mathcal{J}_{G}^{-1}(\sigma):=\left(G \times \mathcal{J}_{G}^{-1}(\sigma)\right) / G_{\sigma},
$$


where the action $A_{\sigma}$ of $G_{\sigma}$ on $G \times \mathcal{J}_{G}^{-1}(\sigma)$ is the twisted action

$$
\begin{aligned}
A_{\sigma}: G_{\sigma} \times\left(G \times \mathcal{J}_{G}^{-1}(\sigma)\right) & \rightarrow G \times \mathcal{J}_{G}^{-1}(\sigma) \\
(h,(g, m)) & \mapsto\left(g h^{-1}, h \cdot m\right) .
\end{aligned}
$$

Remark 6.4. The properness of the action of $G_{\sigma}$ on $G \times \mathcal{J}_{G}^{-1}(\sigma)$ follows from the properness of the action of $G_{\sigma}$ on $\mathcal{J}_{G}^{-1}(\sigma)$.

Proof. Define $F: G \times_{G_{\sigma}} \mathcal{J}_{G}^{-1}(\sigma) \rightarrow G \cdot \mathcal{J}_{G}^{-1}(\sigma)$ by $F\left([g, x]_{G_{\sigma}}\right)=g x \in G \cdot \mathcal{J}_{G}^{-1}(\sigma)$. The map $F$ is well defined. To see this, note that if $[g, x]_{G_{\sigma}}=\left[g^{\prime}, x^{\prime}\right]_{G_{\sigma}}$, then there exists $h \in G_{\sigma}$ such that $g^{\prime}=g h^{-1}$ and $x^{\prime}=h x$. But then we have $F\left(\left[g^{\prime}, x^{\prime}\right]_{G_{\sigma}}\right)=g^{\prime} x^{\prime}=g h^{-1} h x=g x=F\left([g, x]_{G_{\sigma}}\right)$.

The inverse of the function $F$ is given by $F^{-1}: G \cdot \mathcal{J}_{G}^{-1}(\sigma) \rightarrow G \times{ }_{G_{\sigma}} \mathcal{J}_{G}^{-1}(\sigma), F^{-1}(g$. $x)=[g, x]_{G_{\sigma}}$, for any $g \cdot x \in G \cdot \mathcal{J}_{G}^{-1}(\sigma)$. Indeed, $F^{-1}$ is well defined since $g x=g^{\prime} x^{\prime}$ for $x, x^{\prime} \in \mathcal{J}_{G}^{-1}(\sigma)$ and $g, g^{\prime} \in G$ implies $g^{-1} g^{\prime} \in G_{\sigma}$ by definition of $G_{\sigma}$ and hence $\left[g^{\prime}, x^{\prime}\right]_{G_{\sigma}}=$ $\left[g^{\prime}\left(g^{-1} g^{\prime}\right)^{-1},\left(g^{-1} g^{\prime}\right) x^{\prime}\right]_{G_{\sigma}}=[g, x]_{G_{\sigma}}$. We have obviously $F \circ F^{-1}=\operatorname{Id}_{G \cdot \mathcal{J}_{G}^{-1}(\sigma)}$ and $F^{-1} \circ F=$ $\operatorname{Id}_{G \times G_{\sigma} \mathcal{J}_{G}^{-1}(\sigma)}$.

It remains hence to show that $F$ and $F^{-1}$ are smooth functions. We use the commutative diagram

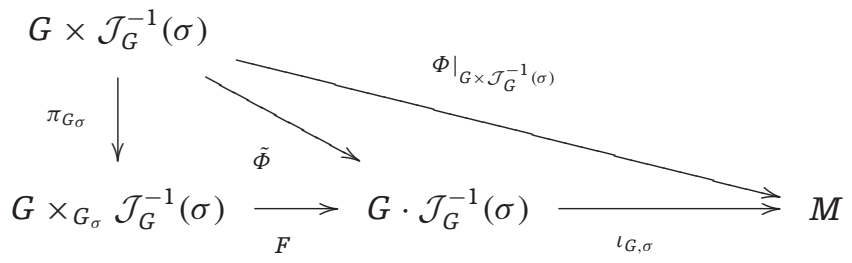

for the smoothness of $F$. Recall that $G \cdot \mathcal{J}_{G}^{-1}(\sigma)$ is an initial submanifold of $M$ because it is a leaf of the integrable tangent distribution $\mathcal{D}_{G}+\mathcal{V}$, that is, the inclusion $\iota_{G, \sigma}$ is regular. The map $\left.\Phi\right|_{G \times \mathcal{J}_{G}^{-1}(\sigma)}=\Phi \circ\left(\operatorname{Id}_{G} \times \iota_{\sigma}\right): G \times \mathcal{J}_{G}^{-1}(\sigma) \rightarrow M$ is smooth. Since its image is $G \cdot \mathcal{J}_{G}^{-1}(\sigma)$, the map $\tilde{\Phi}: G \times \mathcal{J}_{G}^{-1}(\sigma) \rightarrow G \cdot \mathcal{J}_{G}^{-1}(\sigma)$ defined by $\tilde{\Phi}(g, m):=\Phi(g, m)$ for all $g \in G, m \in \mathcal{J}_{G}^{-1}(\sigma)$, is well defined and it is smooth because $\iota_{G, \sigma}$ is regular. Therefore, $F$ is smooth by the properties of the quotient map $\pi_{G_{\sigma}}$.

To show that $F^{-1}$ is smooth, we shall prove that $\operatorname{dim}\left(G \times{ }_{G_{\sigma}} \mathcal{J}_{G}^{-1}(\sigma)\right)=\operatorname{dim}(G$. $\left.\mathcal{J}_{G}^{-1}(\sigma)\right)$ and that

$$
T_{\pi_{G_{\sigma}}(g, m)}\left(\iota_{G, \sigma} \circ F\right): T_{\pi_{G_{\sigma}}(g, m)}\left(G \times_{G_{\sigma}} \mathcal{J}_{G}^{-1}(\sigma)\right) \rightarrow T_{l_{G, \sigma}(F(g, m))} M
$$


is injective. Indeed, if this is known, then $F$ is a bijective smooth map which is a local diffeomorphism, hence a diffeomorphism.

Since $\mathcal{J}_{G}^{-1}(\sigma)$ is a leaf of $\mathcal{D}_{G}$, we have $\operatorname{dim} \mathcal{J}_{G}^{-1}(\sigma)=\operatorname{dim} \mathcal{D}_{G}(m)$, for any $m \in$ $\mathcal{J}_{G}^{-1}(\sigma)$. Therefore, since the $G_{\sigma}$-action on $G \times \mathcal{J}_{G}^{-1}(\sigma)$ is free and proper, using (15), we have

$$
\begin{aligned}
\operatorname{dim}\left(G \times_{G_{\sigma}} \mathcal{J}_{G}^{-1}(\sigma)\right) & =\operatorname{dim} \mathcal{D}_{G}(m)+\operatorname{dim} G-\operatorname{dim} G_{\sigma} \\
& \stackrel{(15)}{=} \operatorname{dim} \mathcal{D}_{G}(m)+\operatorname{dim} G-\operatorname{dim}\left(\mathcal{V}(m) \cap \mathcal{D}_{G}(m)\right)-\operatorname{dim} G_{m} \\
& =\operatorname{dim}\left(\mathcal{D}_{G}(m)+\mathcal{V}(m)\right)-\operatorname{dim} \mathcal{V}(m)+\operatorname{dim} G-\operatorname{dim} G_{m} \\
& =\operatorname{dim}\left(\mathcal{D}_{G}(m)+\mathcal{V}(m)\right)=\operatorname{dim}\left(G \cdot \mathcal{J}_{G}^{-1}(\sigma)\right) .
\end{aligned}
$$

Next we show the injectivity of (20). Let $T_{(g, n)} \pi_{G_{\sigma}}\left(v_{g}, w_{n}\right) \in T_{\pi_{G \sigma}(g, n)}\left(G \times_{G_{\sigma}} \mathcal{J}_{G}^{-1}(\sigma)\right)$ be such that $T_{\pi_{G_{\sigma}}(g, n)} F\left(T_{(g, n)} \pi_{G_{\sigma}}\left(v_{g}, w_{n}\right)\right)=0$. From the diagram it follows that $0=T_{(g, n)} \Phi\left(v_{g}, w_{n}\right)=$ $T_{g} \Phi^{n}\left(v_{g}\right)+T_{n} \Phi_{g}\left(w_{n}\right)$, where $\Phi^{n}(h):=h \cdot n$, for all $h \in G$. Therefore, choosing $\xi \in \mathfrak{g}$ such that $v_{g}=T_{e} L_{g} \xi$, where $L_{g}(h):=g h$, for all $h \in G$, we have

$$
w_{n}=-T_{g \cdot n} \Phi_{g^{-1}}\left(T_{g} \Phi^{n}\left(T_{e} L_{g} \xi\right)\right)=-T_{e}\left(\Phi^{n} \circ L_{g^{-1}} \circ L_{g}\right)(\xi)=-\xi_{M}(n)
$$

Hence $w_{n}=-\xi_{M}(n) \in \mathcal{V}(n) \cap \mathcal{D}_{G}(n)$ which implies that $\xi \in \mathfrak{g}_{\sigma}$ by (15). Thus, $\exp (t \xi) \in G_{\sigma}$ for small $|t|$ and we get

$$
T_{(g, n)} \pi_{G_{\sigma}}\left(v_{g}, w_{n}\right)=\left.\frac{\mathrm{d}}{\mathrm{d} t}\right|_{t=0} \pi_{G_{\sigma}}(g \exp (t \xi), \exp (-t \xi) \cdot n)=0
$$

which proves the injectivity of (20).

A leaf $G \cdot \mathcal{J}_{G}^{-1}(\sigma)$ of $\mathcal{D}_{G}+\mathcal{V}$ is contained in $M_{(H)}$, where $H \subseteq G$ is the compact subgroup such that $\mathcal{J}_{G}^{-1}(\sigma) \subseteq M_{H}$. The induced action of $G$ on $G \cdot \mathcal{J}_{G}^{-1}(\sigma)$ has hence conjugated isotropy subgroups. Using the fact that the topology on $G \cdot \mathcal{J}_{G}^{-1}(\sigma)$ is stronger that the topology induced on it by the topology of $M$, it is easy to show that the action of $G$ on $G \cdot \mathcal{J}_{G}^{-1}(\sigma)$ is proper.

We have the following Dirac Optimal Orbit Reduction Theorem, which is proved in the same manner as Theorems 6.1 and 6.2. 
Theorem 6.5. Let $\sigma \in M / \mathcal{D}_{G}$. The orbit space $M_{\sigma}^{G}:=\left(G \cdot \mathcal{J}_{G}^{-1}(\sigma)\right) / G$ is a regular quotient manifold such that the projection $\pi: G \cdot \mathcal{J}^{-1}(\sigma) \rightarrow M_{\sigma}^{G}$ is a smooth submersion. Define $\omega_{\sigma}^{G} \in \Omega^{2}\left(M_{\sigma}^{G}\right)$ by

$$
\left(\pi^{*} \omega_{\sigma}^{G}\right)(m)\left(v_{m}, w_{m}\right)=\alpha(Y)\left(\iota_{\sigma, G}(m)\right)=-\beta(X)\left(\iota_{\sigma, G}(m)\right)
$$

for any $m \in \mathcal{J}^{-1}(\sigma)$ and any $X, Y \in \Gamma\left(\mathcal{D}_{G}\right)$ defined on an open set around $\iota_{\sigma, G}(m)$, where $(X, \alpha),(Y, \beta) \in \Gamma\left(\mathrm{D} \cap\left(\mathcal{T}_{G} \oplus \mathcal{V}_{G}^{\circ}\right)\right)$ are such that $T_{m} \iota_{\sigma, G} v_{m}=\left(X+V_{1}\right)\left(\iota_{\sigma, G}(m)\right)$ and $T_{m} \iota_{\sigma, G} w_{m}=$ $\left(Y+V_{2}\right)\left(\iota_{\sigma, G}(m)\right)$ for some smooth sections $V_{1}, V_{2} \in \Gamma(\mathcal{V})$.

Then $\left(M_{\sigma}^{G}, \omega_{\sigma}^{G}\right)$ is a presymplectic manifold. The pair $\left(M_{\sigma}^{G}, \mathrm{D}_{\sigma}^{G}\right)$ is called the Dirac optimal orbit reduced space of (M, D) at $\sigma$, where $\mathrm{D}_{\sigma}^{G}$ is the graph of the presymplectic form $\omega_{\sigma}^{G}$.

\subsection{Comparison of the three methods}

Next, we show that if the hypotheses of Theorems 6.1, 6.2, 6.5 hold, then the three methods yield the same reduced objects.

Theorem 6.6. Choose $m \in M$ and set $\sigma:=\mathcal{J}_{G}(m), \rho:=\mathcal{J}(m)$. Assume that the three optimal reduced Dirac manifolds $\left(M_{\sigma}, \mathrm{D}_{\sigma}\right),\left(M_{(\rho)}, \mathrm{D}_{(\rho)}\right)$, and $\left(M_{\sigma}^{G}, \mathrm{D}_{\sigma}^{G}\right)$ are defined. The reduced presymplectic spaces $\left(M_{\sigma}, \omega_{\sigma}\right),\left(M_{(\rho)}, \omega_{(\rho)}\right)$, and $\left(M_{\sigma}^{G}, \omega_{\sigma}^{G}\right)$ are presymplectomorphic.

Remark 6.7. We will show in Theorem 7.1 that the reduced presymplectic spaces correspond also to the presymplectic leaves of the reduced spaces obtained by performing standard Dirac reduction as in [18]. In this paper, it is shown that a Dirac manifold (M, D) that is invariant under a proper $G$-action induces a subspace of the set $\mathfrak{X}(M / G) \times \Omega^{1}(M / G)$ consisting of "vector fields" and "1-forms"on the stratified space $M / G$, whose values define Dirac structures on the strata. To get them, the restrictions of the Dirac structure to the orbit-type manifolds are considered and a regularity condition on $\mathrm{D} \cap\left(\mathcal{T} \oplus \mathcal{V}_{G}^{\circ}\right)$ is postulated. This extends to Dirac manifolds the method used in [25] for Poisson manifolds: if a Poisson manifold is invariant under a proper Lie group action, then there is an induced Poisson bracket on the set of differentiable functions on $M / G$, that restrict to usual Poisson brackets on the strata.

In the case of Poisson manifolds, there is an alternative singular reduction method presented in [12] (see also [15]). If $(M,\{\cdot, \cdot\})$ is a Poisson manifold with a Lie group $G$ acting in a proper canonical way on it, then there is an induced Poisson structure on each isotropy type manifold $M_{H}$, that is invariant under the induced action of $N(H) / H$; here, $H$ is an isotropy subgroup of the action and $N(H)$ is the normalizer of $H$ in $G$. 
Therefore, these Poisson structures descend to the quotient $M_{H} /(N(H) / H)$ whose connected components are strata in $M / G$.

In the paper [17], we show that one can also do singular Dirac reduction in this spirit. We prove that there are induced Dirac structures on the isotropy-type manifolds, invariant under the induced actions of the Lie groups $N(H) / H$, that project (under a regularity condition as in [18]) hence to reduced Dirac structures on the strata of $M / G$. The resulting reduced Dirac structures are identical to the Dirac structures obtained as in [18].

This result is not surprising since each orbit-type manifold is just the $G$-orbit of the corresponding isotropy-type manifold; thus, one should not expect additional information about a $G$-invariant Dirac structures by working on the orbit type rather than on the isotropy type manifolds. Note that in the Poisson case, one can show by using the diffeomorphisms on the strata of $M / G$ that there are also induced Poisson structures on the orbit-type manifolds in $M$, but it seems that this cannot be shown in a direct manner.

The result in the previous theorem just shows that the same kind of phenomenon exists in the Dirac optimal reduction process. By Theorem 3.8, all the reduced presymplectic spaces obtained here and the ones obtained as the presymplectic leaves in $[17,18]$ coincide.

Proof of Theorem 6.6. Define the maps

$$
\begin{aligned}
\Psi: M_{\sigma}^{G} & \longrightarrow M_{\sigma} \\
\left(\pi \circ \pi_{G_{\sigma}}\right)(g, m) & \longmapsto \pi_{\sigma}(m)
\end{aligned} \quad \text { and } \begin{aligned}
\Theta: M_{\sigma} & \longrightarrow M_{\sigma}^{G} \\
\pi_{\sigma}(m) & \longmapsto
\end{aligned}
$$

by the following commutative diagrams.

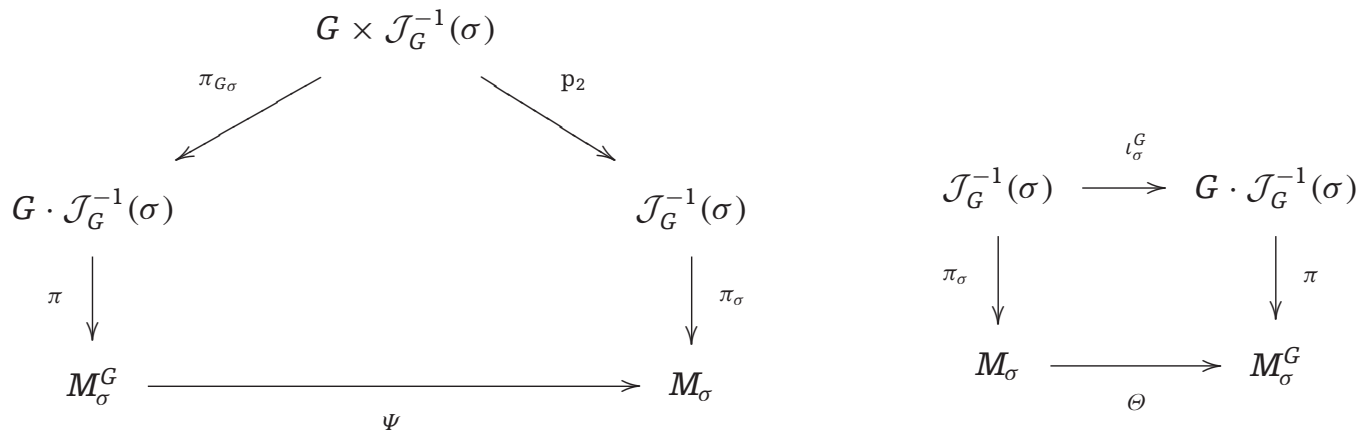


We use the diagrams to check that the maps $\Psi$ and $\Theta$ are inverses of each other and hence both bijective. Indeed, for all $(g, m) \in G \times \mathcal{J}_{G}^{-1}(\sigma)$, we have

$$
(\Theta \circ \Psi)\left(\left(\pi \circ \pi_{G_{\sigma}}\right)(g, m)\right)=\Theta\left(\pi_{\sigma}(m)\right)=\left(\pi \circ \iota_{\sigma}^{G}\right)(m)=\pi(g \cdot m)=\left(\pi \circ \pi_{G_{\sigma}}\right)(g, m),
$$

and, for all $m \in \mathcal{J}_{G}^{-1}(\sigma)$,

$$
(\Psi \circ \Theta)\left(\pi_{\sigma}(m)\right)=\Psi\left(\pi \circ \iota_{\sigma}^{G}(m)\right)=\Psi\left(\left(\pi \circ \pi_{G_{\sigma}}\right)(g, m)\right)=\pi_{\sigma}(m) .
$$

The diagrams are also used to show that both maps are smooth. The equality

$$
\Psi \circ \pi \circ \pi_{G_{\sigma}}=\pi_{\sigma} \circ \mathrm{p}_{2}
$$

shows that $\Psi$ is smooth, since $\pi_{\sigma} \circ \mathrm{p}_{2}$ is smooth and $\pi \circ \pi_{G_{\sigma}}$ is a smooth open map. The equality

$$
\Theta \circ \pi_{\sigma}=\pi \circ \iota_{\sigma}^{G}
$$

shows that $\Theta$ is smooth since $\pi_{\sigma}$ is a smooth open map and $\pi \circ \iota_{\sigma}^{G}$ is smooth.

We define in the same manner the maps

$$
\begin{aligned}
& \Lambda: M_{(\rho)} \quad \longrightarrow \quad M_{\sigma}^{G} \quad \text { and } \Phi: M_{\sigma} \longrightarrow M_{(\rho)} \\
& \pi_{(\rho)}(m) \longmapsto(\pi \circ \iota)(m) \quad \text { and } \quad \pi_{\sigma}(m) \longmapsto\left(\pi_{(\rho)} \circ \iota_{\sigma, \rho}\right)(m)
\end{aligned}
$$

by the following commutative diagrams:
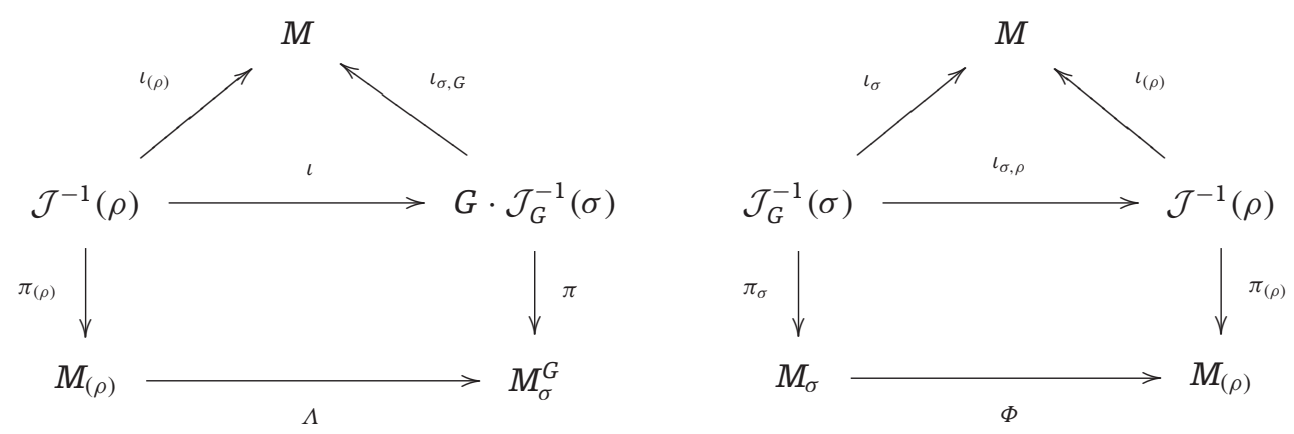
124 M. M. Jotz and T. S. Ratiu

We have then the commutative diagram:

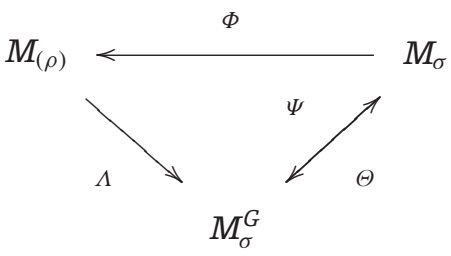

which shows that $\Phi$ and $\Lambda$ are bijective. We have $\Lambda^{-1}=\Phi \circ \Psi$ and $\Phi^{-1}=\Psi \circ \Lambda$. Thus, we have only to show that $\Lambda$ and $\Phi$ are smooth. But using the commutative diagrams, we get $\Lambda \circ \pi_{(\rho)}=\pi \circ \iota$. In addition, using $\iota_{\sigma, G} \circ \iota=\iota_{(\rho)}$ and smoothness of $\iota_{(\rho)}$, we conclude the smoothness of $\iota$ since $\iota_{\sigma, G}$ is a regular immersion. The map $\pi \circ \iota$ is consequently smooth and $\Lambda$ is thus smooth because $\pi_{(\rho)}$ is a smooth open map.

Analogously, we have $\Phi \circ \pi_{\sigma}=\pi_{(\rho)} \circ \iota_{\sigma, \rho}$. An argument similar to the one above shows that the inclusion $\iota_{\sigma, \rho}$ is smooth. Thus, $\Phi$ is smooth, using the fact that $\pi_{\sigma}$ is a smooth open map.

Finally, we prove the equalities

$$
\Phi^{*} \omega_{(\rho)}=\omega_{\sigma} \quad \text { and } \quad \Lambda^{*} \omega_{\sigma}^{G}=\omega_{(\rho)}
$$

which immediately imply that $\Theta$ preserves the presymplectic forms:

$$
\Theta^{*} \omega_{\sigma}^{G}=\left(\Psi^{-1}\right)^{*} \omega_{\sigma}^{G}=(\Lambda \circ \Phi)^{*} \omega_{\sigma}^{G}=\Phi^{*} \Lambda^{*} \omega_{\sigma}^{G}=\Phi^{*} \omega_{(\rho)}=\omega_{\sigma}
$$

Choose $m \in \mathcal{J}_{G}^{-1}(\sigma)$ and vectors $v, w \in T_{m} \mathcal{J}_{G}^{-1}(\sigma)$. Then there exist $G$-invariant descending sections $(X, \alpha)$ and $(Y, \beta)$ of $\mathrm{D}$ such that $T_{m} \iota_{\sigma} v=X\left(\iota_{\sigma}(m)\right)$ and $T_{m} \iota_{\sigma} w=Y\left(\iota_{\sigma}(m)\right)$. We have

$$
\left(\pi_{\sigma}^{*} \Phi^{*} \omega_{(\rho)}\right)(m)(v, w)=\left(\iota_{\sigma, \rho}^{*} \pi_{(\rho)}^{*} \omega_{(\rho)}\right)(m)(v, w)=\left(\pi_{(\rho)}^{*} \omega_{(\rho)}\right)\left(\iota_{\sigma, \rho}(m)\right)\left(T_{m} \iota_{\sigma, \rho} v, T_{m} \iota_{\sigma, \rho} w\right) .
$$

Since

$$
T_{\iota_{\sigma, \rho}(m)} \iota_{(\rho)} T_{m} \iota_{\sigma, \rho} v=T_{m} \iota_{\sigma} v=X\left(\iota_{\sigma}(m)\right)=X\left(\left(\iota_{(\rho)} \circ \iota_{\sigma, \rho}\right)(m)\right)
$$

and

$$
T_{l_{\sigma, \rho}(m)} \iota_{(\rho)} T_{m} \iota_{\sigma, \rho} w=T_{m} \iota_{\sigma} v=Y\left(\iota_{\sigma}(m)\right)=Y\left(\left(\iota_{(\rho)} \circ \iota_{\sigma, \rho}\right)(m)\right),
$$


formula (16) and the fact that $(X, \alpha)$ and $(Y, \beta)$ are descending sections of $D$ imply

$$
\left(\pi_{\sigma}^{*} \Phi^{*} \omega_{(\rho)}\right)(m)(v, w)=\left(\alpha(Y) \circ \iota_{(\rho)}\right)\left(\iota_{\sigma, \rho}(m)\right)=\left(\alpha(Y) \circ \iota_{\sigma}\right)(m) \stackrel{(17)}{=}\left(\pi_{\sigma}^{*} \omega_{\sigma}\right)(m)(v, w),
$$

that is, $\pi_{\sigma}^{*} \Phi^{*} \omega_{(\rho)}=\pi_{\sigma}^{*} \omega_{\sigma}$. Since $\pi_{\sigma}$ is a smooth surjective submersion, the equality $\Phi^{*} \omega_{(\rho)}=\omega_{\sigma}$ is proved.

Next, we prove the equality $\Lambda^{*} \omega_{\sigma}^{G}=\omega_{(\rho)}$. Choose $m \in \mathcal{J}^{-1}(\rho)$ and $v, w \in T_{m} \mathcal{J}^{-1}(\rho)$. Then there exist descending sections $(X, \alpha)$ and $(Y, \beta)$ of $\mathrm{D}$ defined on a neighborhood of $\iota_{(\rho)}(m)$ in $M$ such that $T_{m} \iota_{(\rho)} v=X\left(\iota_{(\rho)}(m)\right)$ and $T_{m} \iota_{(\rho)} w=Y\left(\iota_{(\rho)}(m)\right)$. Since $X$ and $Y$ are descending vector fields, they can be written $X=X^{G}+V$ and $Y=Y^{G}+W$ with $X^{G}, Y^{G} \in$ $\mathfrak{X}(M)^{G}$ and $V, W \in \Gamma(\mathcal{V})$. Assume that $(X, \alpha)$ and $(Y, \beta)$ are defined on a whole tube $U$ for the action of $G$ at $\iota_{(\rho)}(m)$; otherwise, we multiply $(X, \alpha)$ and $(Y, \beta)$ with a bump function that is equal to 1 on a neighborhood $U_{1} \subsetneq U$ of $\iota_{(\rho)}(m)$, and equal to 0 outside from a neighborhood $U_{2}$ of $\iota_{(\rho)}(m)$ such that $U_{1} \subsetneq U_{2} \subsetneq U$.

Consider the $G$-invariant averages $\left(X_{G}, \alpha_{G}\right),\left(Y_{G}, \beta_{G}\right) \in \Gamma(\mathrm{D})$ of $(X, \alpha)$ and $(Y, \beta)$ at $\iota_{(\rho)}(m)=: n$. We have, with $H=G_{n}$

$$
X_{G}(n)=\int_{H}\left(T_{n} \Phi_{h^{-1}} X^{G}(n)+T_{n} \Phi_{h^{-1}} V(n)\right) \mathrm{d} h=X^{G}(n)+V_{G}(n)=X(n)+\left(V_{G}-V\right)(n)
$$

and

$$
\alpha_{G}(n)=\int_{H} \alpha(h \cdot n) \circ T_{n} \Phi_{h} \mathrm{~d} h=\alpha(n) .
$$

Hence, the sections $\left(X_{G}, \alpha_{G}\right)$ and $\left(Y_{G}, \beta_{G}\right)$ are $G$-invariant descending sections of D such that

$$
\begin{aligned}
T_{\iota(m) \iota_{\sigma, G}\left(T_{m} \iota v\right)} & =T_{m} \iota_{(\rho)} v=X\left(\iota_{(\rho)}(m)\right) \\
& =X(n)=X_{G}(n)+\left(V-V_{G}\right)(n)=\left(X_{G}+\left(V-V_{G}\right)\right)\left(\iota_{\sigma, G}(\iota(m))\right)
\end{aligned}
$$

and analogously

$$
T_{\iota(m)} \iota_{\sigma, G}\left(T_{m} \iota w\right)=\left(Y_{G}+\left(W-W_{G}\right)\right)\left(\iota_{\sigma, G}(\iota(m))\right) .
$$


We get, using this and definitions (21) and (16),

$$
\begin{aligned}
\left(\pi_{(\rho)}^{*} \Lambda^{*} \omega_{\sigma}^{G}\right)(m)(v, w) & =\left(\iota^{*} \pi^{*} \omega_{\sigma}^{G}\right)(m)(v, w)=\left(\pi^{*} \omega_{\sigma}^{G}\right)(\iota(m))\left(T_{m} \iota v, T_{m} \iota w\right) \\
& \stackrel{(21)}{=}-\beta_{G}\left(X_{G}\right)\left(\iota_{\sigma, G}(\iota(m))\right)=-\beta_{G}\left(X_{G}+\left(V-V_{G}\right)\right)\left(\iota_{(\rho)}(m)\right) \\
& =-\beta_{G}(n)\left(X_{G}(n)+\left(V-V_{G}\right)(n)\right)=-\beta(n)(X(n)) \\
& =-\beta(X)\left(\iota_{(\rho)}(m)\right) \stackrel{(16)}{=}\left(\pi_{(\rho)}^{*} \omega_{(\rho)}\right)(m)(v, w)
\end{aligned}
$$

that is, $\pi_{(\rho)}^{*} \Lambda^{*} \omega_{\sigma}^{G}=\pi_{(\rho)}^{*} \omega_{(\rho)}$. Since $\pi_{(\rho)}$ is a smooth surjective submersion, we conclude $\Lambda^{*} \omega_{\sigma}^{G}=\omega_{(\rho)}$.

\subsection{Reduction of dynamics}

In this subsection, we write $\{\cdot, \cdot\}$ for the Poisson bracket $\{\cdot, \cdot\}_{D}$ on the admissible functions of ( $M, \mathrm{D})$. We assume that the hypotheses of the preceding subsections are satisfied and study the reduction of dynamics.

Theorem 6.8. Let ( $M, \mathrm{D})$ be a smooth Dirac manifold with a proper Lie group action, such that the orbit optimal momentum map $\mathcal{J}$ is defined. Choose $m \in M$ such that $G_{(\rho)}$ acts properly on $\mathcal{J}^{-1}(\rho)$, where $\rho=\mathcal{J}(m)$. Let $h \in C^{\infty}(M)^{G}$ be a $G$-invariant admissible smooth function on $M$ defined on a neighborhood $U$ of $m$ in $M$. Then:

1. There exists a $G$-invariant vector field $X_{h}$ defined on $U$ such that $\left(X_{h}, \mathrm{~d} h\right)$ is a ( $G$-invariant descending) section of $\mathrm{D}$.

2. The flow $\phi$ of $X_{h}$ commutes with the $G$-action and leaves $\mathcal{J}^{-1}(\rho)$ invariant. Thus, it restricts to a flow $\tilde{\phi}$ on $\mathcal{J}^{-1}(\rho)$, that is, with $\phi_{t} \circ \iota_{(\rho)}=\iota_{(\rho)} \circ \tilde{\phi}_{t}$ for all $t \in \mathbb{R}$ where the left-hand side is defined. The flow $\tilde{\phi}$ commutes then with the $G_{(\rho)}$-action and induces therefore a flow $\phi^{(\rho)}$ on $M_{(\rho)}$ uniquely determined by the relation $\pi_{(\rho)} \circ \tilde{\phi}_{t}=\phi_{t}^{(\rho)} \circ \pi_{(\rho)}$ for all $t \in \mathbb{R}$ where the left-hand side is defined.

3. The vector field $X_{h}^{(\rho)}$ defined by the flow $\phi^{(\rho)}$ on $M_{(\rho)}$ is a section of $\mathrm{G}_{1}^{(\rho)}$; more precisely, we have

$$
\left(X_{h}^{(\rho)}, \mathrm{d} h_{(\rho)}\right) \in \Gamma\left(\mathrm{D}_{(\rho)}\right),
$$

where $h_{(\rho)}$ is the smooth function on $M_{(\rho)}$ defined by $h_{(\rho)} \circ \pi_{(\rho)}=h \circ \iota_{(\rho)}$. 
4. Let $k \in C^{\infty}(M)^{G}$ be another admissible function, and $\{\cdot, \cdot\}_{(\rho)}$ the bracket on admissible functions on $M_{(\rho)}$ defined by $\mathrm{D}_{(\rho)}$. Then we have

$$
(\{h, k\})_{(\rho)}=\left\{h_{(\rho)}, k_{(\rho)}\right\}_{(\rho)},
$$

where the function $(\{h, k\})_{(\rho)}$ is defined by

$$
(\{h, k\})_{(\rho)} \circ \pi_{(\rho)}=\{h, k\} \circ \iota_{(\rho)} .
$$

This makes sense because $\{h, k\}=-X_{h}(k)$ is $G$-invariant.

Proof. Since $h$ is admissible, there exists a vector field $X$ such that $(X, \mathrm{~d} h)$ is a section of D. The 1 -form $\mathrm{d} h$ is a $G$-invariant section of $\mathcal{V}^{\circ}$. Consider the $G$-invariant average $\left(X_{G},(\mathrm{~d} h)_{G}\right)$ of $(X, \mathrm{~d} h)$ in a tube for the action of $G$ centered at the point $m$. Since $\mathrm{d} h$ is $G$-invariant, we have $\mathrm{d} h=(\mathrm{d} h)_{G}$. Set $X_{h}:=X_{G}$; then $\left(X_{h}, \mathrm{~d} h\right)$ is a $G$-invariant descending section of D.

Hence, we have $X_{h} \in \Gamma\left(\mathcal{D}_{G}\right) \subset \Gamma(\mathcal{D})$ and, consequently, the leaves $\mathcal{J}^{-1}(\rho)$ and $\mathcal{J}_{G}^{-1}\left(\mathcal{J}_{G}(m)\right)$ of $\mathcal{D}$ and $\mathcal{D}_{G}$ are left invariant by the flow $\phi$ of $X_{h}$; thus, we can define the restriction $\tilde{\phi}_{t}$ of $\phi_{t}$ to $\mathcal{J}^{-1}(\rho)$ by $\iota_{(\rho)} \circ \tilde{\phi}_{t}=\phi_{t} \circ \iota_{(\rho)}$. Since $X_{h}$ is $G$-invariant, $\phi_{t}$ commutes with the $G$-action and, consequently, $\tilde{\phi}_{t}$ commutes with the $G_{\rho}$-action. Define $\phi_{t}^{(\rho)}$ on $M_{(\rho)}$ by $\pi_{(\rho)} \circ \tilde{\phi}_{t}=\phi_{t}^{(\rho)} \circ \pi_{(\rho)}$ for all $t$ where the left-hand side is defined.

Let $X_{h}^{(\rho)}$ be the vector field defined by the flow $\phi^{(\rho)}$. Then we have

$$
T \pi_{(\rho)} \tilde{X}_{h}=X_{h}^{(\rho)} \circ \pi_{(\rho)},
$$

where $\tilde{X}_{h}$ is the vector field on $\mathcal{J}^{-1}(\rho)$, that is, $\iota_{(\rho)}$-related to $X_{h}$ (i.e., $\tilde{X}_{h}$ is the vector field defined by the flow $\tilde{\phi}$ on $\left.\mathcal{J}^{-1}(\rho)\right)$. For any $n \in \mathcal{J}^{-1}(\rho)$, where $h \circ \iota_{(\rho)}$ is defined, and for any $\tilde{X} \in \mathfrak{X}\left(\mathcal{J}^{-1}(\rho)\right)$ with flow $\phi^{\tilde{X}}$ defined on a neighborhood of $n$, there exists $X \in \Gamma(\mathcal{D})$ with flow $\phi^{X}$ such that $T \iota_{(\rho)} \circ \tilde{X}=X \circ \iota_{(\rho)}$. We compute

$$
\begin{aligned}
\left(\mathrm{i}_{\left.X_{h}^{(\rho)} \omega_{(\rho)}\right)\left(\pi_{(\rho)}(n)\right)\left(T_{n} \pi_{(\rho)} \tilde{X}(n)\right)}=\omega_{(\rho)}\left(\pi_{(\rho)}(n)\right)\left(X_{h}^{(\rho)}\left(\pi_{(\rho)}(n)\right), T_{n} \pi_{(\rho)} \tilde{X}(n)\right)\right. \\
=\omega_{(\rho)}\left(\pi_{(\rho)}(n)\right)\left(T_{n} \pi_{(\rho)} \tilde{X}_{h}(n), T_{n} \pi_{(\rho)} \tilde{X}(n)\right) \\
=\left(\pi_{(\rho)}^{*} \omega_{(\rho)}\right)(n)\left(\tilde{X}_{h}(n), \tilde{X}(n)\right) \\
\stackrel{(16)}{=} \mathbf{d} h\left(\iota_{(\rho)}(n)\right)\left(X\left(\iota_{(\rho)}(n)\right)\right)=\mathbf{d} h\left(\iota_{(\rho)}(n)\right)\left(T_{n} \iota_{(\rho)} \tilde{X}_{(n))}\right.
\end{aligned}
$$




$$
\begin{aligned}
& =\mathbf{d}\left(\iota_{(\rho)}^{*} h\right)(n)(\tilde{X}(n))=\mathbf{d}\left(\pi_{(\rho)}^{*} h_{(\rho)}\right)(n)(\tilde{X}(n)) \\
& =\mathbf{d} h_{(\rho)}\left(\pi_{(\rho)}(n)\right)\left(T_{n} \pi_{(\rho)} \tilde{X}(n)\right) .
\end{aligned}
$$

Hence, we have shown the equality $\mathrm{i}_{X_{h}^{(\rho)}} \omega_{(\rho)}=\mathrm{d} h_{(\rho)}$ and, by the definition of $\mathrm{D}_{(\rho)}$, we get

$$
\left(X_{h}^{(\rho)}, \mathrm{d} h_{(\rho)}\right) \in \Gamma\left(\mathrm{D}_{(\rho)}\right)
$$

which yields also the fact that $h_{(\rho)} \in C^{\infty}\left(M_{(\rho)}\right)$ is admissible.

We show the last statement of the theorem in the same manner. Let $X_{k}$ be the $G$-invariant vector field such that $\left(X_{k}, \mathrm{~d} k\right)$ is a $G$-invariant descending section of $\mathrm{D}$. Then we have

$$
\begin{aligned}
\left\{h_{(\rho)}, k_{(\rho)}\right\}_{(\rho)} \circ \pi_{(\rho)} & =-\left(\mathrm{d} h_{(\rho)}\left(X_{k}^{(\rho)}\right)\right) \circ \pi_{(\rho)}=-\left(\omega_{(\rho)} \circ \pi_{(\rho)}\right)\left(X_{h}^{(\rho)} \circ \pi_{(\rho)}, X_{k}^{(\rho)} \circ \pi_{(\rho)}\right) \\
& =-\left(\pi_{(\rho)}^{*} \omega_{(\rho)}\right)\left(\tilde{X}_{h}, \tilde{X}_{k}\right)=-(\mathrm{d} h)\left(X_{k}\right) \circ \iota_{(\rho)}=\{h, k\} \circ \iota_{(\rho)} \\
& =:(\{h, k\})_{(\rho)} \circ \pi_{(\rho)} .
\end{aligned}
$$

Remark 6.9. As can be easily seen from this proof, an analogous theorem is true for the optimal reduced Dirac spaces $\left(M_{\sigma}, \mathrm{D}_{\sigma}\right)$ and $\left(M_{\sigma}^{G}, \mathrm{D}_{\sigma}^{G}\right)$, if they are defined.

\section{Comparison of Optimal and Standard Dirac Reduction}

In this section, we compare the reduced Dirac manifolds obtained by the standard reduction method in [18] with those obtained by optimal reduction, under the assumption that all necessary conditions on the smooth generalized distribution $\mathrm{D} \cap\left(\mathcal{T} \oplus \mathcal{V}_{G}^{\circ}\right)$ are satisfied. Since we know that the three optimal reduction methods are equivalent if they are all possible, we only consider the optimal (orbit type) point reduction method in this section.

We assume again that $\mathrm{D} \cap\left(\mathcal{T} \oplus \mathcal{V}_{G}^{\circ}\right)$ is spanned pointwise by its descending sections and that $\mathcal{D}$ is integrable, and we let $\mathcal{J}: M \rightarrow M / \mathcal{D}$ be the corresponding orbit optimal momentum map. Since $\mathrm{D} \cap\left(\mathcal{T} \oplus \mathcal{V}_{G}^{\circ}\right)$ is spanned pointwise by the descending sections of $D$, the Dirac structure $D$ induces a Dirac structure on each stratum of the quotient space $M / G$ (Theorem 2.3). The following theorem gives the relation between the strata of $M / G$ endowed with these reduced structures and the reduced manifolds 
$\left(M_{(\rho)}, \mathrm{D}_{(\rho)}\right)$ given by the (orbit type) Dirac optimal reduction theorem (under the assumption that $G_{(\rho)}$ acts properly on $\left.\mathcal{J}^{-1}(\rho)\right)$.

Theorem 7.1. Let $(M, \mathrm{D})$ be a smooth Dirac manifold that is symmetric under the proper action of a Lie group $G$ on $M$. Assume that $D \cap\left(\mathcal{T} \oplus \mathcal{V}_{G}^{\circ}\right)$ is spanned pointwise by its descending sections and that $\mathcal{D}$ is integrable. Let $m \in \mathcal{J}^{-1}(\rho)$, for some $\rho \in M / \mathcal{D}$ such that $G_{(\rho)}$ acts properly on $\mathcal{J}^{-1}(\rho)$. Then, if $P$ is the connected component through $m$ of the orbit-type manifold $M_{\left(G_{m}\right)}$, we have $\mathcal{J}^{-1}(\rho) \subseteq P$. The reduced manifold $M_{(\rho)}$ is diffeomorphic to the presymplectic leaf $\bar{N}$ through $\pi(m)$ of the reduced Dirac manifold $\left(\bar{P}, \mathrm{D}_{\bar{P}}\right)$, where $\bar{P}=\pi(P)$ is the stratum of $M / G$ through $\pi(m)$, via the map $\Pi: M_{(\rho)} \rightarrow \bar{N}, \pi_{(\rho)}(x) \mapsto$ $\left(\pi \circ \iota_{(\rho)}\right)(x)$. Furthermore, $\Pi^{*} \omega_{\bar{N}}=\omega_{(\rho)}$, where $\omega_{\bar{N}}$ is the presymplectic form on $\bar{N}$.

Note that, as already stated in Remark 6.7, the reduced presymplectic manifolds obtained by optimal reduction correspond then also to the presymplectic leaves of the reduced Dirac manifolds as in [17].

Proof of Theorem 7.1. We begin by showing that the map $\Pi$ is well defined. Let $x, y \in$ $\mathcal{J}^{-1}(\rho)$ be such that $\pi_{(\rho)}(x)=\pi_{(\rho)}(y)$. Then there exists $g \in G_{(\rho)} \subseteq G$ such that $\Phi_{g}^{(\rho)}(x)=y$ which implies that $\Phi_{g}\left(\iota_{(\rho)}(x)\right)=\iota_{(\rho)}(y)$ and $\pi\left(\iota_{(\rho)}(X)\right)=\pi\left(\iota_{(\rho)}(y)\right)$. Thus, it remains to show that $\pi\left(\iota_{(\rho)}(x)\right) \in \bar{N}$. Since $x \in \mathcal{J}^{-1}(\rho)$, and by definition of the integral leaves of $\mathcal{D}$, the points $\iota_{(\rho)}(m)$ and $\iota_{(\rho)}(x)$ can be joined by a broken path consisting of finitely many pieces of integral curves of descending sections of $\mathcal{D}$ belonging to descending pairs of $\mathrm{D}$. To simplify the notation, we shall write in what follows simply $x^{\prime}$ for $\iota_{(\rho)}(x)$ and $m^{\prime}$ for $\iota_{(\rho)}(m)$. Assume, without loss of generality, that one such curve suffices, that is, that $x^{\prime}=\phi_{t}\left(m^{\prime}\right)$, where $\phi$ is the flow of a vector field $X \in \Gamma(\mathcal{D})$ for which there exists $\alpha \in \Omega^{1}(M)$ such that $(X, \alpha)$ is a descending section of $\mathrm{D}$. Since $X$ is a descending vector field, it can be written as a sum $X=V+X^{G}$ with $X \in \mathfrak{X}(M)^{G}$ and $V \in \Gamma(\mathcal{V})$. Then $\left[X^{G}, V\right]=0$ and we have $\phi_{t}=\phi_{t}^{G} \circ \phi_{t}^{V}=\phi_{t}^{V} \circ \phi_{t}^{G}$, where $\phi_{t}^{G}$ and $\phi_{t}^{V}$ are the flows of $X^{G}$ and $V$, respectively. Let $\bar{\phi}$ be the flow on $\bar{M}$ induced by $\phi$, that is, $\pi \circ \phi_{s}=\pi \circ \phi_{s}^{V} \circ \phi_{s}^{G}=\pi \circ \phi_{s}^{G}=\bar{\phi}_{s} \circ \pi$, for all $s$. This flow $\bar{\phi}$ generates a vector field $\bar{X}$ on $\bar{M}$ such that $X \sim_{\pi} \bar{X}$. Since $(X, \alpha)$ is a descending section of $\mathrm{D} \cap\left(\mathcal{T} \oplus \mathcal{V}_{G}^{\circ}\right)$, we know by the definition of the reduced Dirac structure on $\bar{P}$ that

$$
\left(X_{\bar{P}}, \alpha_{\bar{P}}\right) \in \Gamma\left(\mathrm{D}_{\bar{P}}\right),
$$

where $X_{\bar{P}}$ is the restriction to $\bar{P}$ of $\bar{X}$ and $\alpha_{\bar{P}}$ is the restriction of $\bar{\alpha}$ to $\bar{P}$, the "1-form" $\bar{\alpha} \in \Omega^{1}(\bar{M})$ being such that $\pi^{*} \bar{\alpha}=\alpha$. Here, we know that $X_{\bar{P}} \in \mathfrak{X}(\bar{P})$ because the flow of $\bar{X}$ 
through points in $\bar{P}$ remains in $\bar{P}$. We have

$$
\left(\bar{\phi}_{t} \circ \pi\right)\left(m^{\prime}\right)=\left(\pi \circ \phi_{t}\right)\left(m^{\prime}\right)=\pi\left(x^{\prime}\right)
$$

which yields, using (22) and the fact that $\left.\bar{\phi}_{t}\right|_{\bar{P}}$ is the flow of $X_{\bar{P}}$, that $\pi\left(l_{(\rho)}(x)\right)$ and $\pi\left(\iota_{(\rho)}(m)\right)$ lie in the same presymplectic leaf $\bar{N}$ of $\left(\bar{P}, \mathrm{D}_{\bar{P}}\right)$. This concludes the proof that $\Pi: M_{\rho} \rightarrow \bar{N}$ is well defined.

To prove that $\Pi$ is injective, choose $x, y \in \mathcal{J}^{-1}(\rho)$ such that $\pi\left(\iota_{(\rho)}(x)\right)=\pi\left(\iota_{(\rho)}(y)\right)$. Then there exists $g \in G$ satisfying

$$
\Phi_{g}\left(\iota_{(\rho)}(x)\right)=\iota_{(\rho)}(y) .
$$

This shows that $g \in G_{\rho}$ and $\Phi_{g}^{(\rho)}(x)=y$, so we get $\pi_{(\rho)}(x)=\pi_{(\rho)}(y)$.

For the surjectivity of $\Pi$ choose $\pi(x) \in \bar{N}$ and assume, again without loss of generality, that

$$
\pi(x)=\phi_{t}^{\bar{P}}\left(\pi\left(\iota_{(\rho)}(m)\right)\right)
$$

where $\phi^{\bar{P}}$ is the flow of some $X_{\bar{P}} \in \mathfrak{X}(\bar{P})$, such that there exists $\alpha_{\bar{P}} \in \Omega^{1}(\bar{P})$ with $\left(X_{\bar{P}}, \alpha_{\bar{P}}\right) \in$ $\Gamma\left(\mathrm{D}_{\bar{P}}\right)$. Choose a descending section $(X, \alpha) \in \Gamma(\mathrm{D})$ and $(\bar{X}, \bar{\alpha}) \in \overline{\mathcal{D}}$ such that $X \sim_{\pi} \bar{X}, \alpha=$ $\pi^{*} \bar{\alpha}$, and $\left.(\bar{X}, \bar{\alpha})\right|_{\bar{P}}=\left(X_{\bar{P}}, \alpha_{\bar{P}}\right)$. The pairs $(X, \alpha)$ and $(\bar{X}, \bar{\alpha})$ exist by the proof of the reduction Theorem 2.3. Then the flows $\phi$ of $X$ and $\bar{\phi}$ of $\bar{X}$ satisfy $\pi \circ \phi_{s}=\bar{\phi}_{s} \circ \pi$ for all $s$ and $\phi_{s}$ restricts to $\mathcal{J}^{-1}(\rho)$ since $X$ is a descending section of $\mathcal{D}$. If we define $x^{\prime} \in \mathcal{J}^{-1}(\rho)$ by $\iota_{(\rho)}\left(x^{\prime}\right)=\phi_{t}\left(\iota_{(\rho)}(m)\right)$, we get, using the fact that $\left.\bar{\phi}_{t}\right|_{\bar{P}}=\phi_{t}^{\bar{P}}$,

$$
\pi\left(\iota_{(\rho)}\left(x^{\prime}\right)\right)=\left(\pi \circ \phi_{t}\right)\left(\iota_{(\rho)}(m)\right)=\left(\bar{\phi}_{t} \circ \pi\right)\left(\iota_{(\rho)}(m)\right)=\pi(x)
$$

and hence $\Pi\left(\pi_{(\rho)}\left(x^{\prime}\right)\right)=\pi(x)$.

Note that we have simultaneously shown that $\pi\left(\mathcal{J}^{-1}(\rho)\right) \subseteq \bar{P}$ is equal, as a set, to $\bar{N}$. Moreover, we claim that the topology of $\bar{N}$ (which is in general not the relative topology induced from the topology on $\bar{P}$ ) is the quotient topology defined by the topology of $\mathcal{J}^{-1}(\rho)$, that is, a set is open in $\bar{N}$ if and only if its preimage under $\pi \circ \iota_{(\rho)}$ is open in $\mathcal{J}^{-1}(\rho)$. This is proved in the following way.

Denote by $\overline{\mathrm{G}}_{1} \subseteq \mathfrak{X}(\bar{M})$ the set of vector fields $\bar{X}$ on $\bar{M}$ such that there exists $\bar{\alpha} \in \Omega^{1}(\bar{M})$ with $(\bar{X}, \bar{\alpha}) \in \overline{\mathcal{D}}$. The presymplectic leaf $\bar{N}$ containing $\bar{m}$ can be seen as the accessible set of $\bar{G}_{1}$ through $\bar{m}$, since $\bar{P}$ is the accessible set through $\bar{m}$ of the family of all vector fields on $\bar{M}$. The topology on $\bar{N}$ is the relative topology induced on $\bar{N}$ by a 
topology we call the $\overline{\mathrm{G}}_{1}$-topology on $\bar{M}$ : this is the strongest topology on $\bar{M}$ such that all the maps

$$
\begin{aligned}
& U \longrightarrow \bar{M}, \\
&\left(t_{1}, \ldots, t_{k}\right) \longmapsto\left(\bar{\phi}_{t_{1}} \bar{X}_{1} \circ \cdots \circ \bar{\phi}_{t_{k}}^{\bar{X}_{k}}\right)(\bar{m})
\end{aligned}
$$

are continuous, where $\bar{m} \in \bar{M}, \bar{\phi}_{t_{i}}^{\bar{X}_{i}}$ is the flow of a vector field $\bar{X}_{i} \in \overline{\mathrm{G}}_{1}$ for $i=1, \ldots, k$, and $U \subseteq \mathbb{R}^{k}$ is an appropriate open set in $\mathbb{R}^{k}$. In the same manner, because $\mathcal{J}^{-1}(\rho)$ is an accessible set of the family

$$
F:=\left\{X \in \mathfrak{X}(M) \mid \exists \alpha \in \Omega^{1}(M) \text { such that }(X, \alpha) \text { is a descending section of } \mathrm{D}\right\},
$$

the topology on $\mathcal{J}^{-1}(\rho)$ is the relative topology induced on $\mathcal{J}^{-1}(\rho)$ by the topology we call the $\mathcal{D}$-topology on $M$ : this is the strongest topology on $M$ such that all the maps

$$
\begin{aligned}
& U \longrightarrow M, \\
&\left(t_{1}, \ldots, t_{k}\right) \longmapsto\left(\phi_{t_{1}}^{X_{1}} \circ \cdots \circ \phi_{t_{k}}^{X_{k}}\right)(m)
\end{aligned}
$$

are continuous, where $m \in M, \phi_{t_{i}}^{X_{i}}$ is the flow of a vector field $X_{i} \in F$, for $i=1, \ldots, k$, and $U \subseteq \mathbb{R}^{k}$ is an appropriate open set in $\mathbb{R}^{k}$.

Now our claim is easy to show, using the fact that, for each section $X_{\bar{P}}$ in $\overline{\mathrm{G}}_{1}$, there exists a descending section $(X, \alpha)$ of $\mathrm{D}$ such that $X \sim_{\pi} \bar{X}$ and hence $\bar{\phi}_{t}^{\bar{X}} \circ \pi=\pi \circ \phi_{t}^{X}$. Conversely, for each descending section $(X, \alpha)$ of $\mathrm{D}$, the vector field $\bar{X}$ satisfying $X \sim_{\pi} \bar{X}$ is an element of $\bar{G}_{1}$ and we have $\bar{\phi}_{t}^{\bar{X}} \circ \pi=\pi \circ \phi_{t}^{X}$. Hence, a map $f: \bar{N} \rightarrow Q$ is smooth if and only if $f \circ\left(\pi \circ \iota_{(\rho)}\right): \mathcal{J}^{-1}(\rho) \rightarrow Q$ is smooth, where $Q$ is an arbitrary smooth manifold. Thus, we have shown that $\bar{N}=\pi\left(\mathcal{J}^{-1}(\rho)\right) \subseteq \bar{P}$ as topological spaces.

Finally, the smoothness of $\Pi$ and of its inverse $\Pi^{-1}: \bar{N} \rightarrow M_{\rho}, \pi\left(\iota_{(\rho)}(x)\right) \mapsto \pi_{\rho}(x)$ follow from the following commutative diagrams:
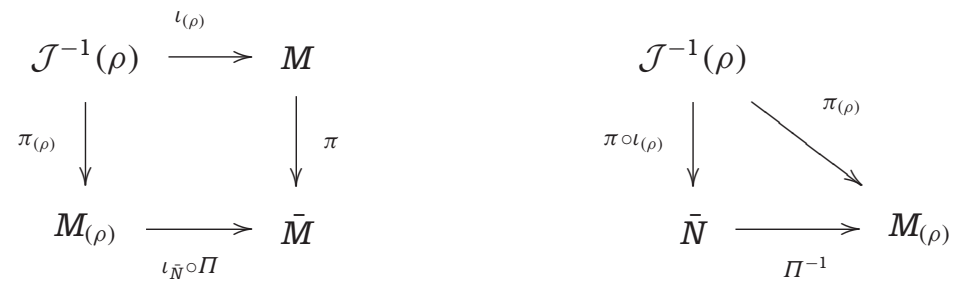
Consider the first diagram. Let $\iota_{\bar{P}, \bar{M}}: \bar{P} \hookrightarrow \bar{M}$ and $\iota_{\bar{N}, \bar{P}}: \bar{N} \hookrightarrow \bar{P}$ be the inclusions. Since $\pi \circ \iota_{(\rho)}$ is smooth, we have automatically (by the quotient manifold structure on $M_{(\rho)}$ ) that $\iota_{\bar{N}} \circ \Pi=\iota_{\bar{P}, \bar{M}} \circ \iota_{\bar{N}, \bar{P}} \circ \Pi$ is smooth. Since $\bar{N}$ is an initial submanifold of $\bar{P}$ and $\bar{P}$ is a stratum of $\bar{M}$, the smoothness of $\Pi$ follows. With the considerations above and, because $\pi_{(\rho)}$ is smooth, we get the smoothness of $\Pi^{-1}$ with the second diagram.

Now we show that $\Pi$ is a presymplectomorphism, that is, $\Pi^{*} \omega_{\bar{N}}=\omega_{\rho}$. Let $\pi_{\rho}(x) \in M_{\rho}, x \in \mathcal{J}^{-1}(\rho)$, and $v, w \in T_{X} \mathcal{J}^{-1}(\rho)$, that is, we have $T_{X} \iota_{(\rho)} v, T_{X} \iota_{(\rho)} w \in \mathcal{D}\left(\iota_{(\rho)}(x)\right)$. Then find $X_{\bar{P}}, Y_{\bar{P}} \in \mathfrak{X}(\bar{P})$ and $\alpha_{\bar{P}}, \beta_{\bar{P}} \in \Omega^{1}(\bar{P})$ such that $\left(X_{\bar{P}}, \alpha_{\bar{P}}\right),\left(Y_{\bar{P}}, \beta_{\bar{P}}\right) \in \Gamma\left(\mathrm{D}_{\bar{P}}\right), T_{X}(\Pi \circ$ $\left.\pi_{(\rho)}\right) v=T_{X}\left(\pi \circ \iota_{(\rho)}\right) v=X_{\bar{P}}\left(\pi\left(\iota_{(\rho)}(X)\right)\right), \quad$ and $\quad T_{X}\left(\Pi \circ \pi_{(\rho)}\right) w=T_{X}\left(\pi \circ \iota_{(\rho)}\right) w=Y_{\bar{P}}\left(\pi\left(\iota_{(\rho)}(X)\right)\right)$. Choose $(\bar{X}, \bar{\alpha}),(\bar{Y}, \bar{\beta}) \in \overline{\mathcal{D}}$ and $X, Y \in \mathfrak{X}(M)$ such that $\left(X, \pi^{*} \bar{\alpha}\right),\left(Y, \pi^{*} \bar{\beta}\right)$ are descending sections of $\mathrm{D}, X \sim_{\pi} \bar{X}, Y \sim_{\pi} \bar{Y}$, and $\left(X_{\bar{P}}, \alpha_{\bar{P}}\right)$ and $\left(Y_{\bar{P}}, \beta_{\bar{P}}\right)$ are the restrictions to $\bar{P}$ of $(\bar{X}, \bar{\alpha})$ and $(\bar{Y}, \bar{\beta})$. Then we get

$$
\begin{aligned}
\left(\Pi^{*} \omega_{\bar{N}}\right)\left(\pi_{(\rho)}(x)\right)\left(T_{X} \pi_{(\rho)} v, T_{X} \pi_{(\rho)} w\right) & =\omega_{\bar{N}}\left(\left(\Pi \circ \pi_{(\rho)}\right)(x)\right)\left(T_{X}\left(\Pi \circ \pi_{(\rho)}\right) v, T_{X}\left(\Pi \circ \pi_{(\rho)}\right) w\right) \\
& =\omega_{\bar{N}}\left(\left(\pi \circ \iota_{(\rho)}\right)(X)\right)\left(T_{X}\left(\pi \circ \iota_{(\rho)}\right) v, T_{X}\left(\pi \circ \iota_{(\rho)}\right) w\right) \\
& =\omega_{\bar{N}}\left(\left(\pi \circ \iota_{(\rho)}\right)(X)\right)\left(X_{\bar{P}}\left(\pi\left(\iota_{(\rho)}(X)\right)\right), Y_{\bar{P}}\left(\pi\left(\iota_{(\rho)}(X)\right)\right)\right) \\
& =\alpha_{\bar{P}}\left(\left(\pi \circ \iota_{(\rho)}\right)(x)\right)\left(Y_{\bar{P}}\left(\pi\left(\iota_{(\rho)}(X)\right)\right)=\alpha\left(\iota_{(\rho)}(X)\right)\left(Y\left(\iota_{(\rho)}(X)\right)\right)\right. \\
& =\omega_{(\rho)}\left(\pi_{(\rho)}(X)\right)\left(T_{X} \pi_{(\rho)} v, T_{X} \pi_{(\rho)} w\right),
\end{aligned}
$$

where the last equality is the definition of $\omega_{(\rho)}$.

\section{Examples}

Example 8.1. We consider the example of the proper action $\Phi$ of $G:=\mathbb{S}^{1} \simeq \mathbb{R} /(2 \pi \mathbb{Z})$ on $M:=\mathbb{R}^{3}$ given by

$$
\Phi_{\alpha}(x, y, z)=\alpha \cdot(x, y, z)=(x \cos \alpha-y \sin \alpha, x \sin \alpha+y \cos \alpha, z) .
$$

The orbit and isotropy types of this action coincide since the Lie group is Abelian. They are $P_{1}=\{0\} \times\{0\} \times \mathbb{R}, P_{1}=M_{H_{1}}$ with $H_{1}=\mathbb{S}^{1}$, and $P_{2}=\mathbb{R}^{3} \backslash P_{1}$, so $P_{2}=M_{H_{2}}$ with $H_{2}=\{e\}$. The orbit of a point $(x, y, z) \in \mathbb{R}^{3}$ is $\left\{\left(x^{\prime}, y^{\prime}, z^{\prime}\right) \in \mathbb{R}^{3} \mid x^{\prime 2}+y^{2}=x^{2}+y^{2}\right.$ and $\left.z^{\prime}=z\right\}$. Thus, the reduced space $\bar{M}$ can be identified with $[0,+\infty) \times \mathbb{R}$ with the projection $\pi$ given by

$$
[0, \infty) \times \mathbb{R} \ni(\bar{x}, \bar{z}):=\pi(x, y, z)=\left(x^{2}+y^{2}, z\right)
$$


It is easy to compute, for each $\alpha \in \mathbb{S}^{1}$,

$$
\Phi_{\alpha}^{*}\left(\partial_{X}\right)=\cos \alpha \partial_{X}-\sin \alpha \partial_{Y}, \quad \Phi_{\alpha}^{*}\left(\partial_{Y}\right)=\sin \alpha \partial_{X}+\cos \alpha \partial_{Y}, \quad \Phi_{\alpha}^{*}\left(\partial_{z}\right)=\partial_{z}
$$

and

$$
\Phi_{\alpha}^{*}(\mathrm{~d} x)=\cos \alpha \mathrm{d} x-\sin \alpha \mathrm{d} y, \quad \Phi_{\alpha}^{*}(\mathrm{~d} y)=\sin \alpha \mathrm{d} x+\cos \alpha \mathrm{d} y, \quad \Phi_{\alpha}^{*}(\mathrm{~d} z)=\mathrm{d} z .
$$

Hence, the Dirac structure D given as the span of the sections

$$
\left(\partial_{X}, d y\right), \quad\left(\partial_{y},-d x\right), \quad\left(\partial_{z}, 0\right)
$$

is $\mathbb{S}^{1}$-invariant, that is, the Lie group $\mathbb{S}^{1}$ acts on $(M, \mathrm{D})$ in a Dirac manner.

In Example A.3, we carried out the explicit computation showing that

$$
\mathcal{T}(m)=\mathcal{T}_{G}(m)=\operatorname{span}_{\mathbb{R}}\left\{\partial_{z}, x \partial_{x}+y \partial_{y}, x \partial_{y}-y \partial_{x}\right\}
$$

for all $m=(x, y) \in \mathbb{R}^{2}$. The set $\mathrm{D} \cap\left(\mathcal{T} \oplus \mathcal{V}_{G}^{\circ}\right)$ is equal to the set $\mathrm{D} \cap\left(\mathcal{T}_{G} \oplus \mathcal{V}_{G}^{\circ}\right)$, since the orbit type manifolds coincide with the isotropy type manifolds, and is spanned by the sections

$$
\left(y \partial_{x}-x \partial_{y}, x \mathrm{~d} x+y \mathrm{~d} y\right) \text { and }\left(\partial_{z}, 0\right) \text { of } \mathrm{D} .
$$

These sections are exact $G$-invariant descending sections of $\mathrm{D}$ and hence $\mathcal{D}_{G}=\mathcal{D}$ is completely integrable. Indeed, it is easy to see that since $\mathcal{D}$ is spanned pointwise by the vector fields $\partial_{z}$ and $y \partial_{x}-x \partial_{y}$, it is integrable and its leaves are the cylinders $M_{r}:=\left\{(x, y, z) \in \mathbb{R}^{3} \mid x^{2}+y^{2}=r\right\}$, for all $r>0$, and the line $\{(0,0, z) \mid z \in \mathbb{R}\}=: M_{0}$.

We identify the leaf space of $\mathcal{D}$ with the closed set $[0, \infty) ; \mathcal{J}(x, y, z)=x^{2}+y^{2}$, for all $(x, y, z) \in \mathbb{R}^{3}$. It is easy to see that $G_{r}=\mathbb{S}^{1}$, for all $r \in[0, \infty)$. The Lie group $\mathbb{S}^{1}$ acts on $M_{r}$ by rotations for $r>0$ and trivially for $r=0$. The reduced spaces are then all lines with trivial presymplectic form.

It has been shown in [18] that the Dirac structures on $\bar{M}_{1}=P_{1} / \mathbb{S}^{1} \simeq \mathbb{R}$ and $\bar{M}_{2}=$ $P_{2} / \mathbb{S}^{1} \simeq \mathbb{R}^{2}$ are given by $D_{\bar{M}_{1}}(\bar{z})=\operatorname{span}\left\{\left(\partial_{\bar{z}}, 0\right)\right\}$ and $D_{\bar{M}_{2}}(\bar{x}, \bar{z})=\operatorname{span}\left\{\left(\partial_{\bar{z}}, 0\right),(0, \bar{x} \mathbf{d} \bar{x})\right\}$. Thus, the symplectic leaves are all lines with trivial Dirac structures and we recover the result of the correspondence theorem. 
Example 8.2. We consider here another example from [18]. Let $M=\mathbb{R}^{3} \times \mathbb{R}^{3}$ with the (automatically proper) diagonal action of $G=\mathbb{S}^{1} \simeq \mathbb{R} /(2 \pi \mathbb{Z})$ on it, that is,

$$
\begin{gathered}
\Phi: \quad \mathbb{S}^{1} \times\left(\mathbb{R}^{3} \times \mathbb{R}^{3}\right) \longrightarrow \mathbb{R}^{3} \times \mathbb{R}^{3} \\
\left.\alpha,\left(\begin{array}{c}
x_{1} \\
y_{1} \\
z_{1}
\end{array}\right),\left(\begin{array}{c}
x_{2} \\
y_{2} \\
z_{2}
\end{array}\right)\right) \longmapsto\left(\left(\begin{array}{c}
x_{1} \cos \alpha-y_{1} \sin \alpha \\
x_{1} \sin \alpha+y_{1} \cos \alpha \\
z_{1}
\end{array}\right),\left(\begin{array}{c}
x_{2} \cos \alpha-y_{2} \sin \alpha \\
x_{2} \sin \alpha+y_{2} \cos \alpha \\
z_{2}
\end{array}\right)\right)
\end{gathered}
$$

The functions

$$
\begin{aligned}
& r_{1}(v, w)=x_{1}^{2}+y_{1}^{2}=\left\|\left(\begin{array}{l}
x_{1} \\
y_{1}
\end{array}\right)\right\|^{2}, \quad r_{2}(v, w)=x_{2}^{2}+y_{2}^{2}=\left\|\left(\begin{array}{l}
x_{2} \\
y_{2}
\end{array}\right)\right\|^{2}, \\
& d(v, w)=x_{1} y_{2}-y_{1} x_{2}=\operatorname{det}\left(\begin{array}{cc}
x_{1} & x_{2} \\
y_{1} & y_{2}
\end{array}\right), \quad s(v, w)=x_{1} x_{2}+y_{1} y_{2}=\left\langle\left(\begin{array}{l}
x_{1} \\
y_{1}
\end{array}\right),\left(\begin{array}{l}
x_{2} \\
y_{2}
\end{array}\right)\right\rangle, \\
& z_{1}(v, w)=z_{1}, \quad z_{2}(v, w)=z_{2},
\end{aligned}
$$

are $\mathbb{S}^{1}$-invariant. They also characterize the $\mathbb{S}^{1}$-orbits of the action since $d$ and $s$ determine in a unique way the angle between the vectors $\left(x_{1}, y_{1}\right)$ and $\left(x_{2}, y_{2}\right)$. Hence, the reduced manifold is the stratified space $\bar{M}=\pi\left(\mathbb{R}^{3} \times \mathbb{R}^{3}\right) \subseteq \mathbb{R}^{6}$, where $\pi: \mathbb{R}^{3} \times \mathbb{R}^{3} \rightarrow \mathbb{R}^{6}$ is given by $\pi(v, w)=\left(r_{1}, r_{2}, d, s, z_{1}, z_{2}\right)(v, w)$. We conclude that $\bar{M}$ is the semi-algebraic set $\bar{M}=\left\{\left(r_{1}, r_{2}, d, s, z_{1}, z_{2}\right) \in \mathbb{R}^{6} \mid r_{1}, r_{2} \geq 0\right.$ and $\left.s^{2}+d^{2}=r_{1} r_{2}\right\}$.

The two strata of $\bar{M}$ are $\bar{M}_{0}=\left\{\left(0,0,0,0, z_{1}, z_{2}\right) \in \mathbb{R}^{6}\right\}$, corresponding to the orbit- (isotropy-)type manifold $M_{\mathbb{S}^{1}}=M_{\left(\mathbb{S}^{1}\right)}=\left\{\left(0,0, z_{1}, 0,0, z_{2}\right) \in \mathbb{R}^{6}\right\}$ with trivial $\mathbb{S}^{1}$-action on it, and $\bar{M}_{1}=\left\{\left(r_{1}, r_{2}, d, s, z_{1}, z_{2}\right) \in \mathbb{R}^{6} \mid\left(r_{1}, r_{2}\right) \neq(0,0)\right.$ and $\left.d^{2}+s^{2}=r_{1} r_{2}\right\}$, corresponding to the orbit- (isotropy-)type manifold $M_{\{0\}}=M_{(\{0\})}=\left\{\left(x_{1}, y_{1}, z_{1}, x_{2}, y_{2}, z_{2}\right) \in \mathbb{R}^{6} \mid\left(x_{1}, y_{1}\right) \neq\right.$ $(0,0)$ or $\left.\left(x_{2}, y_{2}\right) \neq(0,0)\right\}$.

Define $U:=\mathbb{R}_{>0} \times \mathbb{R}^{4} \subset \mathbb{R}^{5}$. Since the points $\left(r_{1}, r_{2}, d, s, z_{1}, z_{2}\right)$ in $\bar{M}_{1}$ satisfy $r_{1}>0$ or $r_{2}>0$, we have two charts for $\bar{M}_{1}$, namely $\left(\psi_{1}(U), \psi_{1}^{-1}\right)$ and $\left(\psi_{2}(U), \psi_{2}^{-1}\right)$, where

$$
\begin{aligned}
& \psi_{1}: \quad \mathbb{R}_{>0} \times \mathbb{R}^{4} \quad \rightarrow \quad \bar{M}_{1} \\
& \left(r_{1}, d, s, z_{1}, z_{2},\right) \mapsto\left(r_{1}, \frac{d^{2}+s^{2}}{r_{1}}, d, s, z_{1}, z_{2}\right), \quad \begin{aligned}
\psi_{1}^{-1}: \psi_{1}(U) \subseteq \bar{M}_{1} & \rightarrow \mathbb{R}_{>0} \times \mathbb{R}^{4} \\
\left(r_{1}, r_{2}, d, s, z_{1}, z_{2}\right) & \mapsto\left(r_{1}, d, s, z_{1}, z_{2}\right)
\end{aligned}
\end{aligned}
$$


and

$$
\begin{aligned}
& \psi_{2}: \quad \mathbb{R}_{>0} \times \mathbb{R}^{4} \quad \rightarrow \quad \bar{M}_{1} \\
& \left(r_{2}, d, s, z_{1}, z_{2}\right) \mapsto\left(\frac{d^{2}+s^{2}}{r_{2}}, r_{2}, d, s, z_{1}, z_{2}\right), \quad \begin{aligned}
\left(r_{1}, r_{2}, d, s, z_{1}, z_{2}\right) & \mapsto \quad\left(r_{2}, d, s, z_{1}, z_{2}\right) .
\end{aligned}
\end{aligned}
$$

Since $\mathcal{V}_{G}^{\circ}(m)=\left\{\mathbf{d} f(m) \mid f \in C^{\infty}(M)^{G}\right\} \quad[18$, Lemma 5.8], we have, for all $m=$ $\left(x_{1}, y_{1}, z_{1}, x_{2}, y_{2}, z_{2}\right) \in \mathbb{R}^{6}$,

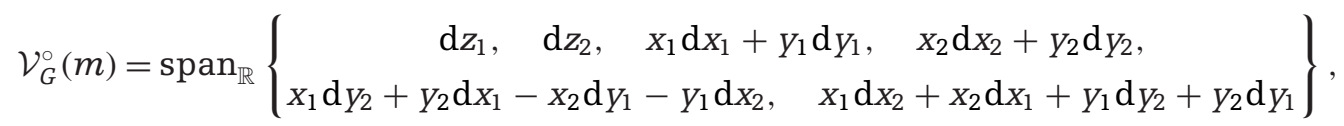

and, as shown in Example A.4,

$$
\mathcal{T}(m)=\mathcal{T}_{G}(m)=\operatorname{span}_{\mathbb{R}}\left\{\begin{aligned}
X_{1}:=\partial_{z_{1}}, & X_{2}:=\partial_{z_{2}}, \\
X_{3}:=x_{1} \partial_{X_{1}}+y_{1} \partial_{Y_{1}}, & X_{4}:=x_{2} \partial_{X_{2}}+y_{2} \partial_{Y_{2}}, \\
X_{5}:=y_{1} \partial_{X_{2}}-x_{1} \partial_{Y_{2}}, & X_{6}:=y_{2} \partial_{X_{1}}-x_{2} \partial_{Y_{1}}, \\
X_{7}:=x_{1} \partial_{X_{2}}+y_{1} \partial_{Y_{2}}, & X_{8}:=x_{2} \partial_{X_{1}}+y_{2} \partial_{Y_{1}}, \\
X_{9}:=x_{1} \partial_{Y_{1}}-y_{1} \partial_{X_{1}}, & X_{10}:=x_{2} \partial_{Y_{2}}-y_{2} \partial_{X_{2}}
\end{aligned}\right\} .
$$

Note that $\mathcal{V}$ is spanned on $M$ by $X_{9}+X_{10}=x_{1} \partial_{Y_{1}}-y_{1} \partial_{X_{1}}+x_{2} \partial_{y_{2}}-y_{2} \partial_{X_{2}}$.

Consider the Dirac structure $\mathrm{D} \subseteq T M \oplus T^{*} M$ spanned by the pairs

$$
\left(\partial_{X_{1}}, d y_{1}\right), \quad\left(\partial_{y_{1}},-d x_{1}\right), \quad\left(\partial_{z_{1}}, 0\right), \quad\left(\partial_{X_{2}},-d y_{2}\right), \quad\left(\partial_{y_{2}}, d x_{2}\right), \quad\left(0, d z_{2}\right) .
$$

Comparing this with the sections of $\mathcal{T}=\mathcal{T}_{G}$ and $\mathcal{V}_{G}^{\circ}$ given above, we find a set $\mathcal{D}^{\mathbb{S}^{1}}$ of exact G-invariant descending sections spanning pointwise the intersection $\mathrm{D} \cap(\mathcal{T} \oplus$ $\left.\mathcal{V}_{G}^{\circ}\right)=\mathrm{D} \cap\left(\mathcal{T}_{G} \oplus \mathcal{V}_{G}^{\circ}\right):$

$$
\begin{aligned}
\mathcal{D}^{\mathbb{S}^{1}}= & \left\{\begin{array}{c}
\left(\partial_{z_{1}}, 0\right),\left(0, \mathrm{~d} z_{2}\right), \\
\left(-x_{1} \partial_{y_{1}}+y_{1} \partial_{X_{1}}, x_{1} \mathrm{~d} x_{1}+y_{1} \mathrm{~d} y_{1}\right),\left(x_{2} \partial_{y_{2}}-y_{2} \partial_{X_{2}}, x_{2} \mathrm{~d} x_{2}+y_{2} \mathrm{~d} y_{2}\right), \\
\left(-x_{1} \partial_{X_{2}}-y_{2} \partial_{y_{1}}-x_{2} \partial_{X_{1}}-y_{1} \partial_{y_{2}}, x_{1} \mathrm{~d} y_{2}+y_{2} \mathrm{~d} x_{1}-x_{2} \mathrm{~d} y_{1}-y_{1} \mathrm{~d} x_{2}\right), \\
\left(x_{1} \partial_{y_{2}}-x_{2} \partial_{y_{1}}-y_{1} \partial_{X_{2}}+y_{2} \partial_{X_{1}}, x_{1} \mathrm{~d} x_{2}+x_{2} \mathrm{~d} x_{1}+y_{1} \mathrm{~d} y_{2}+y_{2} \mathrm{~d} y_{1}\right)
\end{array}\right\} \\
= & \left\{\begin{array}{c}
\left(\partial_{z_{1}}, 0\right),\left(0, \mathrm{~d} z_{2}\right),\left(-X_{9}, \frac{1}{2} \mathrm{~d} r_{1}\right), \\
\left(X_{10}, \frac{1}{2} \mathrm{~d} r_{2}\right),\left(-X_{7}-X_{8}, \mathrm{~d} d\right),\left(X_{6}-X_{5}, \mathrm{~d} s\right)
\end{array}\right\} .
\end{aligned}
$$


Thus, we get, for all $m \in \mathbb{R}^{6}$,

$$
\begin{gathered}
\mathcal{D}(m)=\mathcal{D}_{G}(m)=\operatorname{span}\left\{\begin{array}{c}
\partial_{z_{1}}, \quad X_{9}=x_{1} \partial_{y_{1}}-y_{1} \partial_{X_{1}}, \\
X_{10}=x_{2} \partial_{y_{2}}-y_{2} \partial_{X_{2}}, \\
X_{7}+X_{8}=x_{1} \partial_{X_{2}}+y_{2} \partial_{y_{1}}+x_{2} \partial_{X_{1}}+y_{1} \partial_{Y_{2}}, \\
X_{6}-X_{5}=x_{1} \partial_{y_{2}}-x_{2} \partial_{y_{1}}-y_{1} \partial_{X_{2}}+y_{2} \partial_{X_{1}}
\end{array}\right\}(m) \\
=\left(\operatorname{span}\left\{\mathrm{d} z_{2}, x_{1} \mathrm{~d} x_{1}+y_{1} \mathrm{~d} y_{1}-x_{2} \mathrm{~d} x_{2}-y_{2} \mathrm{~d} y_{2}\right\}\right)^{\circ}=\left(\operatorname{span}\left\{\mathrm{d} z_{2}, \mathrm{~d}\left(r_{1}-r_{2}\right)\right\}\right)^{\circ} .
\end{gathered}
$$

The distribution $\mathcal{D}=\mathcal{D}_{G}$ is hence integrable and its leaf through a point $p=$ $\left(x_{1}, y_{1}, z_{1}, x_{2}, y_{2}, z_{2}\right) \in M$ is

1. $\left\{\left(0,0, t, 0,0, z_{2}\right) \mid t \in \mathbb{R}\right\}$ if $r_{1}(p)=r_{2}(p)=0$,

and otherwise the level set of the functions $z_{2}$ and $r_{1}-r_{2}$ through the point $p=$ $\left(x_{1}, y_{1}, z_{1}, x_{2}, y_{2}, z_{2}\right) \in M$, that is,

2. $\left\{\left(r \cos \alpha, r \sin \alpha, t, r \cos \beta, r \sin \beta, z_{2}\right) \mid r>0, \alpha, \beta, t \in \mathbb{R}\right\}$, if $r_{1}(p)=r_{2}(p)>0$,

3. $\left\{\left(\sqrt{x^{2}+y^{2}+k} \cos \alpha, \sqrt{x^{2}+y^{2}+k} \sin \alpha, t, x, y, z_{2}\right) \mid x, y, \alpha, t \in \mathbb{R}\right\}$, if $k:=\left(r_{1}-r_{2}\right)$ $(p)>0$ and

4. $\left\{\left(x, y, t, \sqrt{x^{2}+y^{2}-k} \cos \alpha, \sqrt{x^{2}+y^{2}-k} \sin \alpha, z_{2}\right) \mid x, y, \alpha, t \in \mathbb{R}\right\}$, if $k:=\left(r_{1}-r_{2}\right)$ $(p)<0$.

The singularity at points where $r_{1}$ and $r_{2}$ both vanish can also be seen considering the flows $\phi^{1}, \phi^{9}, \phi^{10}, \phi^{7+8}, \phi^{6-5}$ of the vector fields $\partial_{z_{1}}, X_{9}, X_{10}, X_{7}+X_{8}, X_{6}-X_{5}$ :

$$
\begin{aligned}
\phi^{1}\left(\left(\begin{array}{l}
x_{1} \\
y_{1} \\
z_{1}
\end{array}\right),\left(\begin{array}{l}
x_{2} \\
y_{2} \\
z_{2}
\end{array}\right)\right) & =\left(\left(\begin{array}{c}
x_{1} \\
y_{1} \\
z_{1}+t
\end{array}\right),\left(\begin{array}{l}
x_{2} \\
y_{2} \\
z_{2}
\end{array}\right)\right), \\
\phi^{9}\left(\left(\begin{array}{l}
x_{1} \\
y_{1} \\
z_{1}
\end{array}\right),\left(\begin{array}{l}
x_{2} \\
y_{2} \\
z_{2}
\end{array}\right)\right) & =\left(\left(\begin{array}{c}
x_{1} \cos t-y_{1} \sin t \\
x_{1} \sin t+y_{1} \cos t \\
z_{1}
\end{array}\right),\left(\begin{array}{l}
x_{2} \\
y_{2} \\
z_{2}
\end{array}\right)\right), \\
\phi^{10}\left(\left(\begin{array}{l}
x_{1} \\
y_{1} \\
z_{1}
\end{array}\right),\left(\begin{array}{l}
x_{2} \\
y_{2} \\
z_{2}
\end{array}\right)\right) & =\left(\left(\begin{array}{c}
x_{1} \\
y_{1} \\
z_{1}
\end{array}\right),\left(\begin{array}{c}
x_{2} \cos t-y_{2} \sin t \\
x_{2} \sin t+y_{2} \cos t \\
z_{2}
\end{array}\right),\right.
\end{aligned}
$$




$$
\begin{aligned}
\phi^{7+8}\left(\left(\begin{array}{l}
x_{1} \\
y_{1} \\
z_{1}
\end{array}\right),\left(\begin{array}{l}
x_{2} \\
y_{2} \\
z_{2}
\end{array}\right)\right) & =\left(\left(\begin{array}{c}
x_{1} \cosh t+x_{2} \sinh t \\
y_{1} \cosh t+y_{2} \sinh t \\
z_{2}
\end{array}\right),\left(\begin{array}{c}
x_{2} \cosh t+x_{1} \sinh t \\
y_{2} \cosh t+y_{1} \sinh t \\
z_{1}
\end{array}\right)\right), \\
\phi^{6-5}\left(\left(\begin{array}{l}
x_{1} \\
y_{1} \\
z_{1}
\end{array}\right),\left(\begin{array}{l}
x_{2} \\
y_{2} \\
z_{2}
\end{array}\right)\right) & =\left(\left(\begin{array}{c}
x_{1} \cosh t+y_{2} \sinh t \\
y_{1} \cosh t-x_{2} \sinh t \\
z_{2}
\end{array}\right),\left(\begin{array}{c}
x_{2} \cosh t-y_{1} \sinh t \\
y_{2} \cosh t+x_{1} \sinh t \\
z_{1}
\end{array}\right)\right) .
\end{aligned}
$$

Hence we can identify the leaf space $M / \mathcal{D}_{G}$ with the set

$$
\left\{\left(r_{1}, r_{2}, r_{1}-r_{2}, z_{2}\right)(p) \mid p \in M\right\} / \sim
$$

where $\sim$ is the equivalence relation given on $\left\{\left(r_{1}, r_{2}, r_{1}-r_{2}, z_{2}\right)(p) \mid p \in M\right\} \subseteq \mathbb{R}_{>0} \times \mathbb{R}_{>0} \times$ $\mathbb{R} \times \mathbb{R}$ by $\left(r_{1}, r_{2}, k, t\right) \sim\left(r_{1}^{\prime}, r_{2}^{\prime}, k^{\prime}, t^{\prime}\right)$ if and only if $t=t^{\prime}$ and

$$
k=k^{\prime} \neq 0
$$

or

$$
\left(k=k^{\prime}=0\right) \quad \text { and } \quad\left(r_{1}>0 \text { or } r_{2}>0\right) \text { and }\left(r_{1}^{\prime}>0 \text { or } r_{2}^{\prime}>0\right)
$$

or

$$
k=k^{\prime}=0 \quad \text { and } \quad r_{1}=r_{2}=r_{1}^{\prime}=r_{2}^{\prime}=0 .
$$

Since $\mathcal{V} \subseteq \mathcal{D}_{G}$, we find $G_{\sigma}=G=\mathbb{S}^{1}$ for all $\sigma \in M / \mathcal{D}_{G}$. The action of $\mathbb{S}^{1}$ on each of the leaves is the restriction to the leaf of the action of $\mathbb{S}^{1}$ on $M$.

We consider the four different cases:

1. If $\sigma=[0,0,0, a] \in M / \mathcal{D}_{G}$, we have $\mathcal{J}_{G}^{-1}(\sigma)=\{(0,0, t, 0,0, a) \mid t \in \mathbb{R}\} \simeq \mathbb{R}$ and the induced action of $\mathbb{S}^{1}$ on $\mathcal{J}_{G}^{-1}(\sigma)$ is trivial. Thus, the reduced space $M_{\sigma}=$ $\mathcal{J}_{G}^{-1}(\sigma) / \mathbb{S}^{1}=\mathcal{J}_{G}^{-1}(\sigma)=\mathbb{R}$ is a line and the presymplectic form is necessarily trivial.

2. If $\sigma=[R, R, 0, a] \in M / \mathcal{D}_{G}$ with $R>0$, we have

$$
\mathcal{J}_{G}^{-1}(\sigma)=\{(r \cos \alpha, r \sin \alpha, t, r \cos \beta, r \sin \beta, a) \mid r>0, \alpha, \beta, t \in \mathbb{R}\}
$$


Hence, if we consider it as a subspace of $M / \mathbb{S}^{1}$, the reduced space is equal to

$$
\begin{aligned}
M_{\sigma}=\mathcal{J}_{G}^{-1}(\sigma) / \mathbb{S}^{1} & =\left\{\left(r^{2}, r^{2}, r^{2} \sin (\alpha-\beta), r^{2} \cos (\alpha-\beta), t, a\right) \mid r>0, \alpha, \beta, t \in \mathbb{R}\right\} \\
& \simeq \mathbb{R}_{>0} \times \mathbb{S}^{1} \times \mathbb{R}
\end{aligned}
$$

via the diffeomorphism

$$
\begin{aligned}
\psi_{\sigma}: M_{\sigma} & \longrightarrow \mathbb{R}_{>0} \times \mathbb{S}^{1} \times \mathbb{R}, \\
\left(r^{2}, r^{2}, r^{2} \sin (\alpha-\beta), r^{2} \cos (\alpha-\beta), t, a\right) & \longmapsto\left(r^{2}, \alpha-\beta, t\right), \\
\psi_{\sigma}^{-1}: \mathbb{R}_{>0} \times \mathbb{S}^{1} \times \mathbb{R} & \longrightarrow M_{\sigma}, \\
(r, \theta, t) & \longmapsto(r, r, r \sin (\theta), r \cos (\theta), t, a) .
\end{aligned}
$$

Let $\pi: M \rightarrow M / \mathbb{S}^{1}$ and $\pi_{\sigma}: \mathcal{J}_{G}^{-1}(\sigma) \rightarrow M_{\sigma}$ be the canonical projections.

We use the coordinates $(r, \theta, t)$ on $\mathbb{R}_{>0} \times \mathbb{S}^{1} \times \mathbb{R}$ and compute the presymplectic form $\omega_{\sigma}$. We have

$$
\begin{aligned}
& \partial_{r} \sim_{\psi_{\sigma}^{-1}} \partial_{r_{1}}+\partial_{r_{2}}+\sin \theta \partial_{s}+\cos \theta \partial_{d}=\frac{2 r_{1} \partial_{r_{1}}+2 r_{2} \partial_{r_{2}}+2 s \partial_{s}+2 d \partial_{d}}{2 r_{1}} \text { and } \\
& \partial_{\theta} \sim_{\psi_{\sigma}^{-1}} s \partial_{d}-d \partial_{s}
\end{aligned}
$$

since $r_{1}=r_{2}$ on $M_{\sigma}$. Since

$$
\begin{aligned}
& X_{1} \partial_{X_{1}}+y_{1} \partial_{y_{1}}+x_{2} \partial_{X_{2}}+y_{2} \partial_{y_{2}} \sim_{\pi} 2 r_{1} \partial_{r_{1}}+2 r_{2} \partial_{r_{2}}+2 s \partial_{s}+2 d \partial_{d} \text { and } \\
& X_{9} \sim_{\pi}-s \partial_{d}+d \partial_{s}
\end{aligned}
$$

this leads to

$$
\begin{aligned}
\omega_{\sigma}\left(\partial_{r}, \partial_{\theta}\right) & =-\frac{1}{2 r_{1}}\left(\pi_{\sigma}^{*} \omega_{\sigma}\right)\left(x_{1} \partial_{X_{1}}+y_{1} \partial_{Y_{1}}+x_{2} \partial_{X_{2}}+y_{2} \partial_{Y_{2}}, X_{9}\right) \\
& =-\frac{1}{2 r_{1}}\left(x_{1} d x_{1}+y_{1} d y_{1}\right)\left(x_{1} \partial_{X_{1}}+y_{1} \partial_{y_{1}}+x_{2} \partial_{X_{2}}+y_{2} \partial_{Y_{2}}\right)=-\frac{1}{2 r_{1}}\left(x_{1}^{2}+y_{1}^{2}\right) \\
& =-\frac{1}{2}
\end{aligned}
$$




$$
\begin{aligned}
& \omega_{\sigma}\left(\partial_{r}, \partial_{t}\right)=\frac{1}{2 r_{1}}\left(\pi_{\sigma}^{*} \omega_{\sigma}\right)\left(x_{1} \partial_{X_{1}}+y_{1} \partial_{y_{1}}+x_{2} \partial_{X_{2}}+y_{2} \partial_{Y_{2}}, \partial_{z_{1}}\right)=0, \quad \text { and } \\
& \omega_{\sigma}\left(\partial_{\theta}, \partial_{t}\right)=\left(\pi_{\sigma}^{*} \omega_{\sigma}\right)\left(-X_{9}, \partial_{z_{1}}\right)=0 .
\end{aligned}
$$

Thus, we find $\omega_{\sigma}(r, \theta, t)=\frac{1}{2} \mathrm{~d} \theta \wedge \mathrm{d} r$.

Note that easy linear algebra arguments show that $x_{1} \partial_{x_{1}}+y_{1} \partial_{y_{1}}+$ $x_{2} \partial_{x_{2}}+y_{2} \partial_{y_{2}}$ is an element of $\mathcal{D}_{G}\left(x_{1}, y_{1}, z_{1}, x_{2}, y_{2}, z_{2}\right)$ if and only if $x_{1}^{2}+y_{1}^{2}=$ $x_{2}^{2}+y_{2}^{2}$, that is, if and only if $\left(x_{1}, y_{1}, z_{1}, x_{2}, y_{2}, z_{2}\right) \in \mathcal{J}_{G}^{-1}(\sigma)$ for $\sigma=[R, R, 0, a]$.

3. If $\sigma=\left[R_{1}, R_{2}, k, a\right] \in M / \mathcal{D}_{G}$ with $k>0$, we have

$$
\mathcal{J}_{G}^{-1}(\sigma)=\left\{\left(\sqrt{x^{2}+y^{2}+k} \cos \alpha, \sqrt{x^{2}+y^{2}+k} \sin \alpha, t, x, y, a\right) \mid x, y, \alpha, t \in \mathbb{R}\right\} .
$$

The reduced space $M_{\sigma}$ is now

$$
\begin{aligned}
M_{\sigma} & =\mathcal{J}_{G}^{-1}(\sigma) / \mathbb{S}^{1} \\
& =\left\{\left(x^{2}+y^{2}+k, x^{2}+y^{2}, d, s, t, a\right) \mid \begin{array}{c}
x, y, t, d, s \in \mathbb{R} \\
d^{2}+s^{2}=\left(x^{2}+y^{2}+k\right)\left(x^{2}+y^{2}\right)
\end{array}\right\} \simeq \mathbb{R}^{3}
\end{aligned}
$$

via the diffeomorphism

$$
\begin{aligned}
\psi_{\sigma}: \quad M_{\sigma} & \longrightarrow \mathbb{R}^{3}, \\
\left(x^{2}+y^{2}+k, x^{2}+y^{2}, d, s, t, a\right) & \longmapsto(d, s, t), \\
\psi_{\sigma}^{-1}: \quad \mathbb{R}^{3} & \longrightarrow M_{\sigma}, \\
(d, s, t) & \longmapsto\left(\frac{\sqrt{k^{2}+4\left(d^{2}+s^{2}\right)}+k}{2},\right. \\
& \left.\frac{\sqrt{k^{2}+4\left(d^{2}+s^{2}\right)}-k}{2}, d, s, t, a\right) .
\end{aligned}
$$


We use the coordinates $(d, s, t)$ on $\mathbb{R}^{3}$ and compute the presymplectic form $\omega_{\sigma}$. We have

$$
\begin{aligned}
& \partial_{d} \sim_{\psi_{\sigma}^{-1}} \frac{2 d}{\sqrt{k^{2}+4\left(s^{2}+d^{2}\right)}} \partial_{r_{1}}+\frac{2 d}{\sqrt{k^{2}+4\left(s^{2}+d^{2}\right)}} \partial_{r_{2}}+\partial_{d} \text { and } \\
& \partial_{s} \sim_{\psi_{\sigma}^{-1}} \frac{2 s}{\sqrt{k^{2}+4\left(s^{2}+d^{2}\right)}} \partial_{r_{1}}+\frac{2 s}{\sqrt{k^{2}+4\left(s^{2}+d^{2}\right)}} \partial_{r_{2}}+\partial_{s} .
\end{aligned}
$$

A computation (see [18]) yields

$$
X_{6}-X_{5} \sim_{\pi} 2 d \partial_{r_{1}}+2 d \partial_{r_{2}}+\left(r_{1}+r_{2}\right) \partial_{d} \text { and } X_{7}+X_{8} \sim_{\pi} 2 s \partial_{r_{1}}+2 s \partial_{r_{2}}+\left(r_{1}+r_{2}\right) \partial_{s} .
$$

With $r_{1}+r_{2}=\sqrt{k^{2}+4\left(s^{2}+d^{2}\right)}$, this leads to

$$
\begin{aligned}
& \omega_{\sigma}\left(\partial_{d}, \partial_{s}\right)=\frac{1}{k^{2}+4\left(s^{2}+d^{2}\right)}\left(\pi_{\sigma}^{*} \omega_{\sigma}\right)\left(X_{6}-X_{5}, X_{7}+X_{8}\right) \\
& =\frac{1}{k^{2}+4\left(s^{2}+d^{2}\right)}\left(x_{1} \mathrm{~d} x_{2}+x_{2} \mathrm{~d} x_{1}+y_{1} \mathrm{~d} y_{2}+y_{2} \mathrm{~d} y_{1}\right) \\
& \times\left(x_{1} \partial_{X_{2}}+y_{2} \partial_{Y_{1}}+x_{2} \partial_{X_{1}}+y_{1} \partial_{Y_{2}}\right) \\
& =\frac{1}{k^{2}+4\left(s^{2}+d^{2}\right)}\left(x_{1}^{2}+x_{2}^{2}+y_{1}^{2}+y_{2}^{2}\right)=\frac{1}{\sqrt{k^{2}+4\left(s^{2}+d^{2}\right)}}, \\
& \omega_{\sigma}\left(\partial_{d}, \partial_{t}\right)=\frac{1}{\sqrt{k^{2}+4\left(s^{2}+d^{2}\right)}}\left(\pi_{\sigma}^{*} \omega_{\sigma}\right)\left(X_{6}-X_{5}, \partial_{z_{1}}\right)=0, \\
& \text { and } \omega_{\sigma}\left(\partial_{s}, \partial_{t}\right)=\frac{1}{\sqrt{k^{2}+4\left(s^{2}+d^{2}\right)}}\left(\pi_{\sigma}^{*} \omega_{\sigma}\right)\left(X_{7}+X_{8}, \partial_{z_{1}}\right)=0 \text {, }
\end{aligned}
$$

which leads to $\omega_{\sigma}(d, s, t)=\frac{1}{\sqrt{k^{2}+4\left(s^{2}+d^{2}\right)}} \mathrm{d} d \wedge \mathrm{d} s$.

4. If $\sigma=\left[R_{1}, R_{2}, k, a\right] \in M / \mathcal{D}_{G}$ with $k<0$, we have

$$
\mathcal{J}_{G}^{-1}(\sigma)=\left\{\left(x, y, t, \sqrt{x^{2}+y^{2}-k} \cos \alpha, \sqrt{x^{2}+y^{2}-k} \sin \alpha, a\right) \mid x, y, \alpha, t \in \mathbb{R}\right\} .
$$


The reduced space $M_{\sigma}$ is then equal to

$$
\begin{aligned}
M_{\sigma} & =\mathcal{J}_{G}^{-1}(\sigma) / \mathbb{S}^{1} \\
& =\left\{\left(x^{2}+y^{2}, x^{2}+y^{2}-k, d, s, t, a\right) \mid \begin{array}{c}
x, y, t, d, s \in \mathbb{R} \\
d^{2}+s^{2}=\left(x^{2}+y^{2}-k\right)\left(x^{2}+y^{2}\right)
\end{array}\right\} \simeq \mathbb{R}^{3}
\end{aligned}
$$

via the diffeomorphism

$$
\begin{aligned}
& \psi_{\sigma}: \quad M_{\sigma} \longrightarrow \mathbb{R}^{3}, \\
&\left(x^{2}+y^{2}, x^{2}+y^{2}-k, d, s, t, a\right) \longmapsto(d, s, t), \\
& \psi_{\sigma}^{-1}: \quad \mathbb{R}^{3} \longrightarrow M_{\sigma}, \\
&(d, s, t) \longmapsto\left(\frac{\sqrt{k^{2}+4\left(d^{2}+s^{2}\right)}+k}{2},\right. \\
&\left.\frac{\sqrt{k^{2}+4\left(d^{2}+s^{2}\right)}-k}{2}, d, s, t, a\right) .
\end{aligned}
$$

We use the coordinates $(d, s, t)$ on $\mathbb{R}^{3}$ and get, in the same manner as above,

$$
\omega_{\sigma}(d, s, t)=\frac{1}{\sqrt{k^{2}+4\left(s^{2}+d^{2}\right)}} \mathrm{d} d \wedge \mathrm{d} s
$$

We want to compare these reduced spaces with the presymplectic leaves of the Dirac structures induced on the two strata $\bar{M}_{0}$ and $\bar{M}_{1}$ of $\bar{M}$ by standard singular reduction. These are given by $\mathrm{D}_{\bar{M}_{0}}(\bar{m})=\operatorname{span}_{\mathbb{R}}\left\{\left(\left.\partial_{z_{1}}\right|_{\bar{m}}, 0\right),\left(0, \mathrm{~d} z_{2}(\bar{m})\right)\right\}$, for all $\bar{m} \in \bar{M}_{0}$, and by

$$
\mathrm{D}_{\bar{M}_{1}}\left(r_{1}, d, s, z_{1}, z_{2}\right)=\operatorname{span}_{\mathbb{R}}\left\{\begin{array}{c}
\left(\partial_{z_{1}}, 0\right),\left(0, \mathrm{~d} z_{2}\right),\left(2 s \partial_{d}-2 d \partial_{s}, \mathrm{~d} r_{1}\right), \\
\left(-2 s \partial_{r_{1}}-\left(r_{1}+\frac{s^{2}+d^{2}}{r_{1}}\right) \partial_{s}, \mathrm{~d} d\right), \\
\left(2 d \partial_{r_{1}}+\left(r_{1}+\frac{s^{2}+d^{2}}{r_{1}}\right) \partial_{d}, \mathrm{~d} s\right)
\end{array}\right\}\left(r_{1}, s, d, z_{1}, z_{2}\right)
$$


in the chart $\left(U, \psi_{1}\right)$ and

$$
\mathrm{D}_{\bar{M}_{1}}\left(r_{2}, s, d, z_{1}, z_{2}\right)=\operatorname{span}_{\mathbb{R}}\left\{\begin{array}{c}
\left(\partial_{z_{1}}, 0\right),\left(0, \mathrm{~d} z_{2}\right),\left(2 s \partial_{d}-2 d \partial_{s}, \mathrm{~d} r_{2}\right), \\
\left(-2 s \partial_{r_{2}}-\left(r_{2}+\frac{s^{2}+d^{2}}{r_{2}}\right) \partial_{s}, \mathrm{~d} d\right), \\
\left(2 d \partial_{r_{2}}+\left(r_{2}+\frac{s^{2}+d^{2}}{r_{2}}\right) \partial_{d}, \mathrm{~d} s\right)
\end{array}\right\}\left(r_{2}, s, d, z_{1}, z_{2}\right)
$$

in the chart $\left(U, \psi_{2}\right)$ (see [18]).

Take $p \in M$. If $p \in M_{\mathbb{S}^{1}}$, that is, $\pi(p) \in \bar{M}_{0}$, we have $p=\left(0,0, z_{1}, 0,0, z_{2}\right)$ and the reduced space $\left(M_{\sigma}, \omega_{\sigma}\right)$ for $\sigma=\mathcal{J}_{G}(p)$ is of the first type: $\left(M_{\sigma}, \omega_{\sigma}\right) \simeq(\mathbb{R}, 0)$. The presymplectic leaf of $\left(\bar{M}_{0}, \mathrm{D}_{\bar{M}_{0}}\right)$ through $\pi(p) \in \bar{M}_{0}$ is obviously $\bar{N}_{p}=\left\{\left(0,0,0,0, t, z_{2}\right) \mid t \in \mathbb{R}\right\} \simeq \mathbb{R}$ with the trivial presymplectic structure. It is easy to see that $\left(M_{\sigma}, \omega_{\sigma}\right) \simeq\left(\bar{N}_{p}, 0\right)$ via the diffeomorphism constructed in the proof of Theorem 7.1.

For $p \in M_{\{e\}}$, we have $\pi(p) \in \bar{M}_{1}$. We study the presymplectic leaves of $\left(\bar{M}_{1}, \mathrm{D}_{\bar{M}_{1}}\right)$. The corresponding distribution $\mathrm{G}_{1}$ is given by

$$
\begin{aligned}
\mathrm{G}_{1}(\pi(p)) & =\operatorname{span}_{\mathbb{R}}\left\{\partial_{z_{1}}, \quad s \partial_{d}-d \partial_{s}, \quad 2 s \partial_{r_{1}}+\left(r_{1}+\frac{s^{2}+d^{2}}{r_{1}}\right) \partial_{s}, \quad 2 d \partial_{r_{1}}+\left(r_{1}+\frac{s^{2}+d^{2}}{r_{1}}\right) \partial_{d}\right\} \\
& =\operatorname{span}_{\mathbb{R}}\left\{\partial_{z_{1}}, 2 s \partial_{r_{1}}+\left(r_{1}+\frac{s^{2}+d^{2}}{r_{1}}\right) \partial_{s}, \quad 2 d \partial_{r_{1}}+\left(r_{1}+\frac{s^{2}+d^{2}}{r_{1}}\right) \partial_{d}\right\}
\end{aligned}
$$

in the chart $\psi_{1}$. We find that $G_{1}$ is the smooth annihilator of the codistribution that is spanned pointwise by $\left\{\mathrm{d} z_{2}, \mathrm{~d}\left(r_{1}-\frac{s^{2}+d^{2}}{r_{1}}\right)\right\}$ and that has constant rank on $\psi_{1}(U) \subseteq \bar{M}_{1}$. With the same argument we find that $\mathrm{G}_{1}$ has constant rank on $\psi_{2}(U) \subseteq \bar{M}_{1}$ and since $\psi_{1}(U) \cap \psi_{2}(U)$ is open and dense in $\bar{M}_{1}$, the distribution $\mathrm{G}_{1}$ is a vector bundle over $\bar{M}_{1}$. We have shown in [18] that it is involutive and so it is completely integrable in the sense of Frobenius. We have again three cases:

1. Suppose that $r_{1}>0,\left(r_{1}-\frac{s^{2}+d^{2}}{r_{1}}\right)(p)=0=k, z_{2}=a \in \mathbb{R}$. Then the leaf $N_{0, a}$ of $\mathrm{G}_{1}$ through $\pi(p)$ is the subset

$$
\begin{aligned}
\bar{M}_{1} \supseteq \psi_{1}(U) \supseteq N_{0, a} & =\left\{\left(r_{1}, s, d, z_{1}, a\right) \mid r_{1}>0, r_{1}^{2}=s^{2}+d^{2}, z_{1} \in \mathbb{R}\right\} \\
& =\left\{(z, z \cos \alpha, z \sin \alpha, t, a) \mid z>0, \alpha \in \mathbb{S}^{1}, t \in \mathbb{R}\right\} \simeq \mathbb{R}_{>0} \times \mathbb{S}^{1} \times \mathbb{R}
\end{aligned}
$$


via the diffeomorphism

$$
\psi_{0, a}: \quad N_{0, a} \longrightarrow \mathbb{R}_{>0} \times \mathbb{S}^{1} \times \mathbb{R}
$$

$(z, z \cos \alpha, z \sin \alpha, t, a) \longmapsto(z, \alpha, t)$,

$$
\psi_{0, a}^{-1}: \quad \mathbb{R}_{>0} \times \mathbb{S}^{1} \times \mathbb{R} \longrightarrow N_{0, a}
$$

$$
(z, \alpha, t) \longmapsto(z, z \cos \alpha, z \sin \alpha, t, a)
$$

Note that this leaf of $\mathrm{G}_{1}$ is included in the intersection $\psi_{1}(U) \cap \psi_{2}(U)$; the values of $r_{1}$ and $r_{2}$ are equal on the leaf. Thus, for instance, if $r_{1}$ vanishes, then $r_{2}$ has to be zero too, which is not possible on $\bar{M}_{1}$. We compute the presymplectic structure on $N_{0, a}$. Since

$$
\begin{aligned}
& \partial_{z} \sim \psi_{0, a}^{-1} \partial_{r_{1}}+\cos \alpha \partial_{s}+\sin \alpha \partial_{d}=\frac{1}{r_{1}}\left(r_{1} \partial_{r_{1}}+s \partial_{s}+d \partial_{d}\right), \\
& \partial_{\alpha} \sim_{\psi_{0, a}^{-1}} s \partial_{d}-d \partial_{s} \quad \text { and } \quad \partial_{t} \sim_{\psi_{0, a}^{-1}} \partial_{z_{1}}
\end{aligned}
$$

we have

$$
\begin{aligned}
\omega_{N_{0, a}}\left(\partial_{t}, \partial_{z}\right) & =0, \quad \omega_{N_{0, a}}\left(\partial_{t}, \partial_{\alpha}\right)=0 \\
\text { and } \quad \omega_{N_{0, a}}\left(\partial_{z}, \partial_{\alpha}\right) & =-\frac{1}{2} \mathrm{~d} r_{1}\left(\frac{1}{r_{1}}\left(r_{1} \partial_{r_{1}}+s \partial_{s}+d \partial_{d}\right)\right)=-\frac{1}{2} .
\end{aligned}
$$

Therefore, $\omega_{N_{0, a}}=\frac{1}{2} \mathrm{~d} \alpha \wedge \mathrm{d} z$. This shows that $\left(N_{0, a}, \omega_{N_{0, a}}\right)$ is presymplectomorphic to $\left(M_{\sigma}, \omega_{\sigma}\right)$, where $\sigma=\mathcal{J}_{G}(p)=\left[r_{1}, r_{1}, 0, a\right]$.

2. Suppose that $r_{1}>0,\left(r_{1}-\frac{s^{2}+d^{2}}{r_{1}}\right)(p)=k>0, z_{2}=a \in \mathbb{R}$. Then the leaf $N_{k, a}$ of $\mathrm{G}_{1}$ through $\pi(p)$ is the subset

$$
\begin{aligned}
\bar{M}_{1} \supseteq \psi_{1}(U) \supseteq N_{k, a} & =\left\{\left(r_{1}, s, d, z_{1}, a\right) \mid r_{1}>0, r_{1}-\frac{s^{2}+d^{2}}{r_{1}}=k, z_{1} \in \mathbb{R}\right\} \\
& =\left\{\left(\frac{\sqrt{4\left(s^{2}+d^{2}\right)+k^{2}}+k}{2}, s, d, z_{1}, a\right) \mid z_{1}, s, d \in \mathbb{R}\right\} .
\end{aligned}
$$

Note that since $r_{1}-r_{2}$ is equal to $k>0$ on $N_{k, a}$, we have $r_{1}>0$ on $N_{k, a}$ and hence, $N_{k, a} \subseteq \psi_{1}(U)$. To compute the presymplectic structure on $N_{k, a}$, we study 
its graph, which is the induced Dirac structure on the leaf (see (5)). We have

$$
\Gamma\left(\mathrm{D}_{N_{k, a}}\right)=\operatorname{span}_{C^{\infty}\left(N_{k, a}\right)}\left\{\begin{array}{c}
\left(\partial_{z_{1}}, 0\right),\left(s \partial_{d}-d \partial_{s}, \frac{s}{\sqrt{4\left(s^{2}+d^{2}\right)+k^{2}}} \mathrm{~d} s\right. \\
\left.+\frac{d}{\sqrt{4\left(s^{2}+d^{2}\right)+k^{2}}} \mathrm{~d} d\right), \\
\left(-\sqrt{4\left(s^{2}+d^{2}\right)+k^{2}} \partial_{s}, \mathrm{~d} d\right), \\
\left(\sqrt{4\left(s^{2}+d^{2}\right)+k^{2}} \partial_{d}, \mathrm{~d} s\right) .
\end{array}\right\}
$$

We have used the fact that since $r_{1}-k=\frac{s^{2}+d^{2}}{r_{1}}$, we have $r_{1}+\frac{s^{2}+d^{2}}{r_{1}}=2 r_{1}-k=$ $\sqrt{4\left(s^{2}+d^{2}\right)+k^{2}}$, and the equality

$$
\begin{aligned}
\mathrm{d} r_{1} & =8 s \cdot \frac{1}{2 \cdot 2 \cdot \sqrt{4\left(s^{2}+d^{2}\right)+k^{2}}} \mathrm{~d} s+8 d \cdot \frac{1}{2 \cdot 2 \cdot \sqrt{4\left(s^{2}+d^{2}\right)+k^{2}}} \mathrm{~d} d \\
& =\frac{2 s}{\sqrt{4\left(s^{2}+d^{2}\right)+k^{2}}} \mathrm{~d} s+\frac{2 d}{\sqrt{4\left(s^{2}+d^{2}\right)+k^{2}}} \mathrm{~d} d .
\end{aligned}
$$

This yields

$$
\Gamma\left(\mathrm{D}_{N_{k, a}}\right)=\operatorname{span}_{C^{\infty}\left(N_{k, a}\right)}\left\{\begin{array}{c}
\left(\partial_{Z_{1}}, 0\right),\left(-\sqrt{4\left(s^{2}+d^{2}\right)+k^{2}} \partial_{s}, \mathrm{~d} d\right), \\
\left(\sqrt{4\left(s^{2}+d^{2}\right)+k^{2}} \partial_{d}, \mathrm{~d} s\right),
\end{array}\right\}
$$

and thus $\omega_{N_{k, a}}=\frac{1}{\sqrt{4\left(s^{2}+d^{2}\right)+k^{2}}} \mathrm{~d} d \wedge \mathrm{d} s$.

3. Suppose that $r_{2}>0,\left(\frac{s^{2}+d^{2}}{r_{2}}-r_{2}\right)(p)=k<0, z_{2}=a \in \mathbb{R}$. Then the leaf $N_{k, a}$ of $\mathrm{G}_{1}$ through $p$ is the subset

$$
\begin{aligned}
\bar{M}_{1} \supseteq \psi_{2}(U) \supseteq N_{k, a} & =\left\{\left(r_{2}, s, d, z_{1}, a\right) \mid r_{2}>0, \frac{s^{2}+d^{2}}{r_{2}}-r_{2}=k, z_{1} \in \mathbb{R}\right\} \\
& =\left\{\left(\frac{\sqrt{4\left(s^{2}+d^{2}\right)+k^{2}}+k}{2}, s, d, z_{1}, a\right) \mid z_{1}, s, d \in \mathbb{R}\right\} .
\end{aligned}
$$

Note that since $r_{1}-r_{2}$ is equal to $k<0$ on $N_{k, a}$, we have $r_{2}>0$ on $N_{k, a}$ and hence, $N_{k, a} \subseteq \psi_{2}(U)$. To compute the presymplectic structure on $N_{k, a}$, we study 
its graph, which is the induced Dirac structure on the leaf (see (5)). We have

$$
\begin{aligned}
& \Gamma\left(\mathrm{D}_{N_{k, a}}\right) \\
& =\operatorname{span}_{C^{\infty}\left(N_{k, a}\right)}\left\{\begin{array}{c}
\left(\partial_{z_{1}}, 0\right), \\
\left.+\frac{d}{\sqrt{4\left(s^{2}+d^{2}\right)+k^{2}}} \mathrm{~d} d\right), \\
\left(-\sqrt{4\left(s^{2}+d^{2}\right)+k^{2}} \partial_{s}, \mathrm{~d} d\right), \\
\left(\sqrt{4\left(s^{2}+d^{2}\right)+k^{2}} \partial_{d}, \mathrm{~d} s\right)
\end{array}\right.
\end{aligned}
$$

as in the preceding case. This yields

$$
\Gamma\left(\mathrm{D}_{N_{k, a}}\right)=\operatorname{span}_{C^{\infty}\left(N_{k, a}\right)}\left\{\begin{array}{c}
\left(\partial_{z_{1}}, 0\right),\left(-\sqrt{4\left(s^{2}+d^{2}\right)+k^{2}} \partial_{s}, \mathrm{~d} d\right), \\
\left(\sqrt{4\left(s^{2}+d^{2}\right)+k^{2}} \partial_{d}, \mathrm{~d} s\right),
\end{array}\right\}
$$

and thus $\omega_{N_{k, a}}=\frac{1}{\sqrt{4\left(s^{2}+d^{2}\right)+k^{2}}} \mathrm{~d} d \wedge \mathrm{d} s$.

\section{Acknowledgements}

The authors thank the referees for comments and suggestions that have improved the exposition.

\section{Funding}

This research was partially supported by the Swiss National Science Foundation grant 200021121512 and by a "Dorothea Schlözer fellowship" from the University of Göttingen (to M.M.J.) and was partially supported by the Swiss National Science Foundation grant 200021-121512 and by the government grant of the Russian Federation for support of research projects implemented by leading scientists, Lomonosov Moscow State University under the agreement No. 11.G34.31.0054 (to T.S.R.)

\section{Appendix 1. Tube Theorem and $G$-invariant Average for Proper Lie Group Actions}

We recall here a construction from [18] which is used in some proofs of this paper.

If the action of a Lie group $G$ on a smooth manifold $M$ is proper, we can find for each point $m \in M$ a $G$-invariant neighborhood of $m$ such that the action can be described easily on this neighborhood. The proof of the following theorem can be found, for example, in [23]. 
Theorem A.1 (Tube Theorem). Let $M$ be a manifold and $G$ a Lie group acting properly on $M$. For a given point $m \in M$ denote $H:=G_{m}$. Then there exists a $G$-invariant open neighborhood $U$ of the orbit $G \cdot m$, called tube at $m$, and a $G$-equivariant diffeomorphism $G \times_{H} B \stackrel{\sim}{\longrightarrow} U$. The set $B$ is an open $H$-invariant neighborhood of 0 in an $H$-representation space $H$-equivariantly isomorphic to $T_{m} M / T_{m}(G \cdot m)$. The $H$ representation on $T_{m} M / T_{m}(G \cdot m)$ is given by $h \cdot\left(v+T_{m}(G \cdot m)\right):=T_{m} \Phi_{h}(v)+T_{m}(G \cdot m)$, $h \in H, v \in T_{m} M$. The smooth manifold $G \times_{H} B$ is the quotient of the smooth free and proper (twisted) action $\Psi$ of $H$ on $G \times B$ given by $\Psi(h,(g, b)):=\left(g h^{-1}, h \cdot b\right), g \in G, h \in H$, $b \in B$. The $G$-action on $G \times_{H} B$ is given by $k \cdot[g, b]:=[k g, b]_{H}$, where $k, g \in G, b \in B$, and $[g, b]_{H} \in G \times{ }_{H} B$ is the equivalence class (i.e., $H$-orbit) of $(g, b)$.

Let $m \in M$ and $H:=G_{m}$. If the action of $G$ on $M$ is proper, the isotropy subgroup $H$ of $m$ is a compact Lie subgroup of $G$. Hence, there exists a Haar measure $\mathrm{d} h$ on $H$, that is, a $G$-invariant measure on $H$ satisfying $\int_{H} \mathrm{~d} h=1$ (see, e.g., [11]). Left $G$-invariance of $\mathrm{d} h$ is equivalent to right $G$-invariance of $\mathrm{d} h$, that is, $R_{g}^{*} \mathrm{~d} h=\mathrm{d} h=L_{g}^{*} \mathrm{~d} h$ for all $g \in H$, where $L_{g}: H \rightarrow H$ (respectively, $R_{g}: H \rightarrow H$ ) denotes left (respectively, right) translation by $g$ on $H$.

Let $X \in \mathfrak{X}(M)$ be defined on the tube $U$ at $m \in M$ of the proper action of the Lie group $G$ on $M$. Using the Tube Theorem, we write the points of $U$ as equivalence classes $[g, b]_{H}$ with $g \in G$ and $b \in B$. The vector field $X_{G}$ is defined by

$$
X_{G}\left([g, b]_{H}\right):=\left(\Phi_{g^{-1}}^{*}\left(\int_{H} \Phi_{h}^{*} X \mathrm{~d} h\right)\right)\left([g, b]_{H}\right)
$$

That is, for each point $m^{\prime}=[g, b]_{H} \in U$, we have

$$
X_{G}\left([g, b]_{H}\right)=T_{[e, b]_{H}} \Phi_{g}\left(\int_{H}\left(T_{[h, b]_{H}} \Phi_{h^{-1}} X\left([h, b]_{H}\right)\right) \mathrm{d} h\right) .
$$

This defines a smooth $G$-invariant vector field $X_{G}$ called the $G$-invariant average of the vector field $X$ (see [18]). Note that $X_{G}$ is, in general, not equal to $X$ (at any point); it can even vanish. Indeed, $G$-invariant vector fields are tangent to the isotropy-type manifolds (see [23]). Hence, if we choose a $G$-invariant Riemannian metric on $M$ and a section $X$ of the (G-invariant) orthogonal $\left.T P^{\perp} \subseteq T M\right|_{P}$ of $T P$ relative to this metric, where $P$ is a stratum of $M$, its $G$-invariant average is both a section of $T P^{\perp}$ and tangent to $P$, so it is the zero section (see also [10, Lemma 2.4]). 
Similarly, the $G$-invariant average $\alpha_{G} \in \Omega^{1}(M)^{G}$ of $\alpha \in \Omega^{1}(M)$ is defined by

$$
\alpha_{G}\left([g, b]_{H}\right):=\left(\Phi_{g^{-1}}^{*}\left(\int_{H} \Phi_{h}^{*} \alpha \mathrm{d} h\right)\right)\left([g, b]_{H}\right),
$$

that is, for each point $m^{\prime}=[g, b]_{H} \in U$, we have

$$
\alpha_{G}\left([g, b]_{H}\right)=\left(\int_{H} \Phi_{h}^{*} \alpha \mathrm{d} h\right)_{[e, b]_{H}} \circ T_{[g, b]_{H}} \Phi_{g^{-1}}=\left(\int_{H}\left(\alpha\left([h, b]_{H}\right) \circ T_{[e, b]_{H}} \Phi_{h}\right) \mathrm{d} h\right) \circ T_{[g, b]_{H}} \Phi_{g^{-1}}
$$

The 1-form $\alpha_{G}$ is well-defined, smooth, and $G$-invariant (see [18]).

If $(X, \alpha)$ is a section of a $G$-invariant generalized distribution $\Delta$, then $\left(X_{G}, \alpha_{G}\right)$ is a $G$-invariant section of $\Delta$.

Note that, in the same manner, we can define the $G$-invariant average $f_{G}$ of a smooth function $f$ defined on the tube $U$ for the action of $G$ at $m$. The function $f_{G}$ is defined by

$$
f_{G}\left([g, b]_{H}\right):=\int_{h \in H} f\left([h, b]_{H}\right) \mathrm{d} h .
$$

\section{Appendix 2. The Module of Equivariant Vector Fields on a Representation Space}

Let $\Phi: G \times V \rightarrow V$ be a finite-dimensional representation of a compact Lie group $G$. We review a method presented in [7] to find $C^{\infty}(V)^{G}$-generators for the set $\mathfrak{X}(V)^{G}:=\{X \in$ $\left.\mathfrak{X}(V) \mid \Phi_{g}^{*} X=X \quad \forall g \in G\right\}$ of $G$-equivariant vector fields on $V$.

Since $G$ is compact, averaging an arbitrary inner product on $V$ yields a $G$ invariant inner product $\langle$,$\rangle on V$. Thus, we can assume, without loss of generality, that the representation $\Phi$ is orthogonal. Denote by $\langle\rangle:, V^{*} \times V \rightarrow \mathbb{R}$ the nondegenerate duality pairing.

The computation of the set of generators for $\mathfrak{X}(V)^{G}$ is facilitated by the following two observations:

(1) There is a bijective correspondence between G-equivariant vector fields on $V$ and G-equivariant maps $V \rightarrow V$.

Indeed, since $T V \simeq V \times V$ and the tangent lift of the representation $\Phi$ to $T V$ is given by $g \cdot(v, w):=T_{v} \Phi_{g} w=\left(\Phi_{g} v, \Phi_{g} w\right)$, for all $g \in G, v, w \in V$, we can associate to each $X \in \mathfrak{X}(V)^{G}$ the smooth $G$-equivariant map $f_{X}: V \rightarrow V$, 
$f_{X}(v):=\operatorname{pr}_{2}(X(v))$, and vice versa; $\mathrm{pr}_{2}: V \times V \rightarrow V$ is the projection on the second factor.

(2) There is a surjective map from the set of G-invariant real-valued functions $C^{\infty}(V \times V)^{G}$ on $V \times V$ to the set $\mathfrak{X}(V)^{G}$ of G-equivariant vector fields on $V$.

Indeed, if $\varphi: T V=V \times V \rightarrow \mathbb{R}$ is a smooth $G$-invariant function, define $f_{\varphi}(v):=$ $\mathbf{d}_{2} \varphi(v, 0) \in V^{*}$ for all $v \in V$, where $\mathbf{d}_{2} \varphi$ denotes the derivative relative to the second variable. Then $f_{\varphi}: V \rightarrow V^{*}$ is a $G$-equivariant function, where the $G$-representation on $V^{*}$ is defined by $g \cdot l:=l \circ \Phi_{g^{-1}}$, for all $l \in V^{*}$ and $g \in G$. The $G$-invariant inner product $\langle$, $\rangle$ on $V$ induces the isomorphism $v \in V \mapsto\langle\langle v, \cdot\rangle\rangle \in V^{*}$ of $G$-representations and hence $f_{\varphi}$ induces a $G$-equivariant map $\tilde{f}_{\varphi}: V \rightarrow V$ defined by $\left\langle\left\langle\tilde{f}_{\varphi}(v), w\right\rangle:=\left\langle f_{\varphi}(v), w\right\rangle=\left\langle\mathbf{d}_{2} \varphi(v, 0), w\right\rangle\right.$ for all $v, w \in V$. Therefore, we get $X_{\varphi} \in \mathfrak{X}(V)^{G}$ defined by $X_{\varphi}(v):=\left(v, \tilde{f}_{\varphi}(v)\right)$ for all $v \in V$.

Conversely, each $X \in \mathfrak{X}(V)^{G}$, uniquely defines the smooth $G$-equivariant map $f_{X}: V \rightarrow V$ given by $X(v)=\left(v, f_{X}(v)\right)$ for all $v \in V$ and hence the smooth $G$-invariant function $\varphi_{X}: V \times V \rightarrow \mathbb{R}$ defined by $\varphi_{X}(v, w)=\left\langle\left\langle f_{X}(v), w\right\rangle\right.$ which is linear in the second component, that is, $\varphi_{X} \in \mathcal{S}(V \times V)^{G}:=\left\{\varphi \in C^{\infty}(V \times V)^{G} \mid \varphi(v, \cdot) \in V^{*}\right.$, for all $\left.v \in V\right\}$. Note that if $\psi \in \mathcal{S}(V \times V)^{G}$, then $\psi(v, w)=\left\langle\mathbf{d}_{2} \psi(v, 0), w\right\rangle$. Using this identity, it is easily seen that the correspondences $\varphi \in \mathcal{S}(V \times V)^{G} \mapsto X_{\varphi} \in \mathfrak{X}(V)^{G}, X \in \mathfrak{X}(V)^{G} \mapsto \varphi_{X} \in \mathcal{S}(V \times V)^{G}$ are inverses to each other.

So we have a bijective map $\mathfrak{X}(V)^{G} \leftrightarrow \mathcal{S}(V \times V)^{G}$. In particular, the map $\varphi \in$ $C^{\infty}(V \times V)^{G} \mapsto X_{\varphi} \in \mathfrak{X}(V)^{G}$ is surjective.

Let $\left\{p_{1}, \ldots, p_{n}\right\}$ be a Hilbert basis for the ring and finitely generated $\mathbb{R}$-algebra of $G$-invariant polynomials on $V \times V$. The Hilbert map $\mathcal{H}: V \times V \rightarrow \mathbb{R}^{n}, \mathcal{H}(v, w):=$ $\left(p_{1}(v, w), \ldots, p_{n}(v, w)\right)$ is proper (inverse images of compact sets are compact), it separates orbits (if $\left(v^{\prime}, w^{\prime}\right) \neq g \cdot(v, w)$ for all $g \in G$, then $\mathcal{H}\left(v^{\prime}, w^{\prime}\right) \neq \mathcal{H}(v, w)$ ), and there is a homeomorphism $\overline{\mathcal{H}}:(V \times V) / G \stackrel{\sim}{\longrightarrow} \mathcal{H}(V \times V) \subseteq \mathbb{R}^{n}$ such that $\overline{\mathcal{H}} \circ \pi=\mathcal{H}$, where $\pi$ : $V \times V \rightarrow(V \times V) / G$ is the projection on the orbit space (see [7, Theorem 5.2.9]). The theorem of Schwarz-Mather (see, for instance, [23, Theorem 2.5.3]) states that for each $G$-invariant function $\varphi \in C^{\infty}(V \times V)^{G}$, there exists $F_{\varphi} \in C^{\infty}\left(\mathbb{R}^{n}\right)$ such that $\varphi=F_{\varphi} \circ$ $\left(p_{1}, \ldots, p_{n}\right)$. Since

$$
f_{\varphi}(v):=\mathbf{d}_{2} \varphi(v, 0)=\sum_{i=1}^{n} \frac{\partial F_{\varphi}}{\partial x_{i}}\left(p_{1}(v, 0), \ldots, p_{n}(v, 0)\right) \mathbf{d}_{2} p_{i}(v, 0) \in V^{*}
$$

the $G$-equivariant vector fields $X_{1}, \ldots, X_{n}, X_{i}(v):=\left(v, \tilde{p}_{i}(v)\right),\left\langle\left\langle\tilde{p}_{i}(v), w\right\rangle\right\rangle=\left\langle\mathbf{d}_{2} p_{i}(v, 0), w\right\rangle$, for all $w \in V, i=1, \ldots, n$, associated to the Hilbert basis $p_{1}, \ldots, p_{n}$ are spanning vector fields for the $C^{\infty}(V)^{G}$-module $\mathfrak{X}(V)^{G}$. 
In the examples below, we need to know the Hilbert basis for the diagonal actions of $\mathbb{S}^{1}$ and $\mathrm{SO}(3)$ on $n$ copies of $\mathbb{R}^{2}$, respectively $\mathbb{R}^{3}$. These bases are given in the following proposition, which is proved in [20, Theorem 10.2].

\section{Proposition A.2.}

1. Consider the diagonal action $\phi$ of $\mathbb{S}^{1}=\mathrm{SO}(2)$ on $n$ copies of $\mathbb{R}^{2}$, that is, $\phi: \mathbb{S}^{1} \times\left(\mathbb{R}^{2}\right)^{n} \mapsto\left(\mathbb{R}^{2}\right)^{n}, \phi\left(\alpha,\left(v_{1}, \ldots, v_{n}\right)\right)=\left(\alpha \cdot v_{1}, \ldots, \alpha \cdot v_{n}\right)$. Write $\mathcal{P}\left(\left(\mathbb{R}^{2}\right)^{n}\right)$ as $\mathbb{R}\left[X_{1}, Y_{1}, \ldots, X_{n}, Y_{n}\right]$ and define $P_{i j}, Q_{i j} \in \mathcal{P}\left(\left(\mathbb{R}^{2}\right)^{n}\right)^{\mathbb{S}^{1}}$ by $P_{i j}=X_{i} X_{j}+Y_{i} Y_{j}$ and $Q_{i j}=X_{i} Y_{j}-X_{j} Y_{i}$ for all $i, j=1, \ldots, n$. Then $\mathcal{B}_{n}:=\left\{P_{i j}, Q_{k l} \mid 1 \leq i \leq j \leq n, 1 \leq\right.$ $k<l \leq n\}$ is a Hilbert basis for $\mathcal{P}\left(\left(\mathbb{R}^{2}\right)^{n}\right)^{\mathbb{S}^{1}}$.

2. Consider the diagonal action $\psi$ of $\mathrm{SO}(3)$ on $n$ copies of $\mathbb{R}^{3}$, that is, $\psi: \mathrm{SO}(3) \times\left(\mathbb{R}^{3}\right)^{n} \mapsto\left(\mathbb{R}^{3}\right)^{n}, \psi\left(A,\left(v_{1}, \ldots, v_{n}\right)\right)=\left(A \cdot v_{1}, \ldots, A \cdot v_{n}\right)$. Write $\mathcal{P}\left(\left(\mathbb{R}^{3}\right)^{n}\right)$ as $\mathbb{R}\left[X_{1}, Y_{1}, Z_{1}, \ldots, X_{n}, Y_{n}, Z_{n}\right]$ and define $P_{i j}, Q_{i j k} \in \mathcal{P}\left(\left(\mathbb{R}^{3}\right)^{n}\right)^{\mathrm{SO}_{(3)}}$ by $P_{i j}=$ $X_{i} X_{j}+Y_{i} Y_{j}+Z_{i} Z_{j}$ and $Q_{i j k}=X_{i} Y_{j} Z_{k}+Y_{i} Z_{j} X_{k}+Z_{i} X_{j} Y_{k}-X_{i} Y_{k} Z_{j}-Y_{i} X_{j} Z_{k}-$ $Z_{i} Y_{j} X_{k}$ for all $i, j, k=1, \ldots, n$. Then $\mathcal{C}_{n}:=\left\{P_{i j}, Q_{k l m} \mid 1 \leq i \leq j \leq n, 1 \leq k<l<\right.$ $m \leq n\}$ is a Hilbert basis for $\mathcal{P}\left(\left(\mathbb{R}^{3}\right)^{n}\right)^{\mathrm{SO}(3)}$.

Example A.3. We consider Example 8.1: the (automatically proper) action $\Phi$ of $G:=$ $\mathbb{S}^{1} \simeq \mathbb{R} /(2 \pi \mathbb{Z})$ on $M:=\mathbb{R}^{3}$ given by

$$
\Phi_{\alpha}(x, y, z)=\alpha \cdot(x, y, z)=(x \cos \alpha-y \sin \alpha, x \sin \alpha+y \cos \alpha, z) .
$$

We want to find $C^{\infty}(M)^{G}$-generators for the $G$-invariant vector fields on $M=$ $\mathbb{R}^{3}, G=\mathbb{S}^{1}$. Hence, we have to find the invariant functions for the diagonal action of $\mathbb{S}^{1}$ on $M \times M$, that is, the action $\Psi: \mathbb{S}^{1} \times\left(\mathbb{R}^{3} \times \mathbb{R}^{3}\right) \rightarrow\left(\mathbb{R}^{3} \times \mathbb{R}^{3}\right)$ given by $\Psi_{\alpha}(v, w)=$ $\left(\Phi_{\alpha}(v), \Phi_{\alpha}(w)\right)$ for all $v, w \in \mathbb{R}^{3}$. Write $(v, w)=\left(x_{v}, y_{v}, z_{v}, X_{w}, y_{w}, z_{w}\right) \in \mathbb{R}^{6}$. Then, by Proposition A.2, the Hilbert basis for $\mathcal{P}^{\mathbb{S}^{1}}\left(\left(\mathbb{R}^{3}\right)^{2}\right)$ is given by $\left\{p_{1}, p_{2}, p_{3}, p_{4}, p_{5}, p_{6}\right\}$, where

$$
\begin{aligned}
& p_{1}(v, w)=x_{v}^{2}+y_{v}^{2} \quad p_{2}(v, w)=x_{w}^{2}+y_{w}^{2} \quad p_{3}(v, w)=z_{v} \\
& p_{4}(v, w)=z_{w} \quad p_{5}(v, w)=X_{v} X_{w}+y_{v} Y_{w} \quad p_{6}(v, w)=X_{v} Y_{w}-y_{v} X_{w} .
\end{aligned}
$$

Since $\mathbf{d}_{w} p_{1}(v, 0)=0, \mathbf{d}_{w} p_{2}(v, 0)=0, \mathbf{d}_{w} p_{3}(v, 0)=0, \mathbf{d}_{w} p_{4}(v, 0)=\mathbf{d} z_{w}, \mathbf{d}_{w} p_{5}(v, 0)=$ $x_{v} \mathbf{d} x_{w}+y_{v} \mathbf{d} y_{w}, \mathbf{d}_{w} p_{6}(v, 0)=x_{v} \mathbf{d} y_{w}-y_{v} \mathbf{d} x_{w}$, the method in [7] reviewed above yields the generators $X_{1}=X_{2}=X_{3}=0, X_{4}(v)=\partial_{Z_{v}}, X_{5}(v)=X_{v} \partial_{X_{v}}+y_{v} \partial_{Y_{v}}, X_{6}(v)=X_{v} \partial_{Y_{v}}-y_{v} \partial_{X_{v}}$ of the 
$C^{\infty}\left(\mathbb{R}^{6}\right)^{\mathbb{S}^{1}}$-module of equivariant vector fields $\mathfrak{X}\left(\mathbb{R}^{6}\right)^{\mathbb{S}^{1}}$ on $\mathbb{R}^{6}$. Note that $X_{6}$ is the fundamental vector field of the action of $\mathbb{S}^{1}$ on $\mathbb{R}^{3}$ defined by the Lie algebra element $1 \in T_{1} \mathbb{S}^{1} \simeq \mathbb{R}$.

Thus, we get $\Gamma(\mathcal{T})=\Gamma\left(\mathcal{T}_{G}\right)=\operatorname{span}_{C^{\infty}(M)}\left\{\partial_{z}, x \partial_{x}+y \partial_{y}, x \partial_{y}-y \partial_{x}\right\}$ as was used in Example 8.1.

Example A.4. We consider here the action of Example 8.2. The vector space $M=\mathbb{R}^{3} \times$ $\mathbb{R}^{3}$ is endowed with the (automatically proper) diagonal action of $G=\mathbb{S}^{1} \simeq \mathbb{R} /(2 \pi \mathbb{Z}$ ) on it, that is,

$$
\begin{gathered}
\Phi: \quad \mathbb{S}^{1} \times\left(\mathbb{R}^{3} \times \mathbb{R}^{3}\right) \longrightarrow \mathbb{R}^{3} \times \mathbb{R}^{3}, \\
\left.\alpha,\left(\begin{array}{l}
x_{1} \\
y_{1} \\
z_{1}
\end{array}\right),\left(\begin{array}{c}
x_{2} \\
y_{2} \\
z_{2}
\end{array}\right)\right) \longmapsto\left(\left(\begin{array}{c}
x_{1} \cos \alpha-y_{1} \sin \alpha \\
x_{1} \sin \alpha+y_{1} \cos \alpha \\
z_{1}
\end{array}\right),\left(\begin{array}{c}
x_{2} \cos \alpha-y_{2} \sin \alpha \\
x_{2} \sin \alpha+y_{2} \cos \alpha \\
z_{2}
\end{array}\right)\right)
\end{gathered}
$$

We have to consider the action $\Psi$ of $\mathbb{S}^{1}$ on $\mathbb{R}^{12} \simeq\left(\mathbb{R}^{3} \times \mathbb{R}^{3}\right) \times\left(\mathbb{R}^{3} \times \mathbb{R}^{3}\right)$ defined by

$$
\begin{aligned}
\Psi: \quad \mathbb{S}^{1} \times\left(\left(\mathbb{R}^{3} \times \mathbb{R}^{3}\right) \times\left(\mathbb{R}^{3} \times \mathbb{R}^{3}\right)\right) & \longrightarrow\left(\left(\mathbb{R}^{3} \times \mathbb{R}^{3}\right) \times\left(\mathbb{R}^{3} \times \mathbb{R}^{3}\right)\right), \\
(v, w, u, t) & \longrightarrow\left(\Phi_{\alpha}(v), \Phi_{\alpha}(w), \Phi_{\alpha}(u), \Phi_{\alpha}(t)\right) .
\end{aligned}
$$

We write $v=\left(x_{v}, y_{v}, z_{v}\right), w=\left(x_{w}, y_{w}, z_{w}\right)$, etc. By Proposition A.2, we have $\mathcal{H}=\left\{p_{i}: \mathbb{R}^{12} \rightarrow\right.$ $\mathbb{R} \mid i=1, \ldots, 20\}$, where

$$
\begin{aligned}
& p_{1}(v, w, u, t)=z_{v}, \quad p_{2}(v, w, u, t)=z_{w}, \quad p_{3}(v, w, u, t)=z_{u}, \quad p_{4}(v, w, u, t)=z_{t}, \\
& p_{5}(v, w, u, t)=x_{v}^{2}+y_{v}^{2}, \quad p_{6}(v, w, u, t)=x_{w}^{2}+y_{w}^{2}, \quad p_{7}(v, w, u, t)=x_{u}^{2}+y_{u}^{2}, \\
& p_{8}(v, w, u, t)=x_{t}^{2}+y_{t}^{2}, \quad p_{9}(v, w, u, t)=x_{v} x_{w}+y_{v} y_{w}, \quad p_{10}(v, w, u, t)=x_{v} x_{u}+y_{v} y_{u}, \\
& p_{11}(v, w, u, t)=x_{v} x_{t}+y_{v} y_{t}, \quad p_{12}(v, w, u, t)=x_{w} X_{u}+y_{w} Y_{u}, \quad p_{13}(v, w, u, t)=x_{w} x_{t}+y_{w} y_{t}, \\
& p_{14}(v, w, u, t)=x_{u} x_{t}+y_{u} y_{t}, \quad p_{15}(v, w, u, t)=x_{v} y_{w}-y_{v} x_{w}, \quad p_{16}(v, w, u, t)=x_{v} Y_{u}-y_{v} x_{u}, \\
& p_{17}(v, w, u, t)=x_{v} y_{t}-y_{v} x_{t}, \quad p_{18}(v, w, u, t)=x_{w} y_{u}-y_{w} x_{u}, \quad p_{19}(v, w, u, t)=x_{w} y_{t}-y_{w} X_{t}, \\
& p_{20}(v, w, u, t)=x_{u} y_{t}-y_{u} x_{t} .
\end{aligned}
$$


Since these functions determine the lengths of the four vectors and the angles between them, we have, as predicted, $\left(p_{1}, \ldots, p_{20}\right)\left(\mathbb{R}^{12}\right) \simeq \mathbb{R}^{12} / \mathbb{S}^{1}$. We compute the vector fields $X_{1}, \ldots, X_{20}$ associated to these polynomial functions in $\mathcal{H}$. Since

$$
\begin{aligned}
& \tilde{p}_{1}(v, w)=0, \quad \tilde{p}_{2}(v, w)=0, \quad \tilde{p}_{3}(v, w)=\mathrm{d} z_{u}, \quad \tilde{p}_{4}(v, w)=\mathrm{d} z_{t}, \\
& \tilde{p}_{5}(v, w)=0, \quad \tilde{p}_{6}(v, w)=0, \quad \tilde{p}_{7}(v, w)=\left.\left(2 x_{u} \mathrm{~d} x_{u}+2 y_{u} \mathrm{~d} y_{u}\right)\right|_{(v, w, 0,0)}=0, \\
& \tilde{p}_{8}(v, w)=\left.\left(2 x_{t} \mathrm{~d} x_{t}+2 y_{t} \mathrm{~d} y_{t}\right)\right|_{(v, w, 0,0)}=0, \quad \tilde{p}_{9}(v, w)=0, \quad \tilde{p}_{10}(v, w)=x_{v} \mathrm{~d} x_{u}+y_{v} \mathrm{~d} y_{u}, \\
& \tilde{p}_{11}(v, w)=x_{v} \mathrm{~d} x_{t}+y_{v} \mathrm{~d} y_{t}, \quad \tilde{p}_{12}(v, w)=x_{w} \mathrm{~d} x_{u}+y_{w} \mathrm{~d} y_{u}, \quad \tilde{p}_{13}(v, w)=x_{w} \mathrm{~d} x_{t}+y_{w} \mathrm{~d} y_{t}, \\
& \tilde{p}_{14}(v, w)=\left.\left(x_{u} \mathrm{~d} x_{t}+x_{t} \mathrm{~d} x_{u}+y_{u} \mathrm{~d} y_{t}+y_{t} \mathrm{~d} x_{u}\right)\right|_{(v, w, 0,0)}=0, \quad \tilde{p}_{15}(v, w)=0, \\
& \tilde{p}_{16}(v, w)=x_{v} \mathrm{~d} y_{u}-y_{v} \mathrm{~d} x_{u}, \tilde{p}_{17}(v, w)=x_{v} \mathrm{~d} y_{t}-y_{v} \mathrm{~d} x_{t}, \\
& \tilde{p}_{18}(v, w)=x_{w} \mathrm{~d} y_{u}-y_{w} \mathrm{~d} x_{u}, \quad \tilde{p}_{19}(v, w)=x_{w} \mathrm{~d} y_{t}-y_{w} \mathrm{~d} x_{t}, \\
& \tilde{p}_{20}(v, w)=\left.\left(x_{u} \mathrm{~d} y_{t}+y_{t} \mathrm{~d} x_{u}-y_{u} \mathrm{~d} x_{t}-x_{t} \mathrm{~d} y_{u}\right)\right|_{(v, w, 0,0)}=0,
\end{aligned}
$$

we get

$$
\begin{gathered}
X_{1}=X_{2}=X_{5}=X_{6}=X_{7}=X_{8}=X_{9}=X_{14}=X_{15}=X_{20}=0 \\
X_{3}(v, w)=\partial_{Z_{v}}, \quad X_{4}(v, w)=\partial_{Z_{w}}, \quad X_{10}(v, w)=X_{v} \partial_{X_{v}}+y_{v} \partial_{Y_{v}}, \\
X_{11}(v, w)=X_{v} \partial_{X_{w}}+y_{v} \partial_{Y_{w}}, \quad X_{12}(v, w)=X_{w} \partial_{X_{v}}+y_{w} \partial_{Y_{v}}, \quad X_{13}(v, w)=X_{w} \partial_{X_{w}}+Y_{w} \partial_{Y_{w}}, \\
X_{16}(v, w)=X_{v} \partial_{Y_{v}}-y_{v} \partial_{X_{v}}, \quad X_{17}(v, w)=X_{v} \partial_{Y_{w}}-y_{v} \partial_{X_{w}}, \\
X_{18}(v, w)=X_{w} \partial_{Y_{v}}-y_{w} \partial_{X_{v}}, \quad \text { and } \quad X_{19}(v, w)=X_{w} \partial_{Y_{w}}-y_{w} \partial_{X_{w}} .
\end{gathered}
$$

Thus, $\mathcal{T}_{G}=\mathcal{T}$ is spanned by

$$
\left\{\begin{array}{c}
X_{3}(v, w)=\partial_{Z_{v}}, \quad X_{4}(v, w)=\partial_{z_{w}}, \quad X_{10}(v, w)=X_{v} \partial_{X_{v}}+y_{v} \partial_{Y_{v}}, \\
X_{11}(v, w)=x_{v} \partial_{X_{w}}+y_{v} \partial_{Y_{w}}, \quad X_{12}(v, w)=x_{w} \partial_{X_{v}}+y_{w} \partial_{Y_{v}}, \quad X_{13}(v, w)=x_{w} \partial_{X_{w}}+y_{w} \partial_{Y_{w}}, \\
X_{16}(v, w)=x_{v} \partial_{Y_{v}}-y_{v} \partial_{X_{v}}, \quad X_{17}(v, w)=X_{v} \partial_{Y_{w}}-y_{v} \partial_{X_{w}}, \\
X_{18}(v, w)=x_{w} \partial_{Y_{v}}-y_{w} \partial_{X_{v}}, \quad X_{19}(v, w)=x_{w} \partial_{Y_{w}}-y_{w} \partial_{X_{w}} .
\end{array}\right\} .
$$

Note that the vertical space of the action is spanned by $V=X_{16}+X_{19}$. 
Example A.5. Our last example is an example in [18], inspired by Bierstone [1]. We consider the diagonal action $\Phi$ of $G:=\mathrm{SO}(3)$ on $M:=\mathbb{R}^{3} \times \mathbb{R}^{3}$, that is, $\Phi: \mathrm{SO}(3) \times\left(\mathbb{R}^{3} \times\right.$ $\left.\mathbb{R}^{3}\right) \rightarrow \mathbb{R}^{3} \times \mathbb{R}^{3}, \Phi(A, v, w):=A \cdot(v, w):=(A v, A w)$.

Here, we thus have to consider the action $\Psi$ of $\mathrm{SO}(3)$ on $\mathbb{R}^{12}$ given by $\Psi(A,(v, w, u, t))=(A v, A w, A u, A t)$. We write again $v=\left(x_{v}, y_{v}, z_{v}\right), w=\left(x_{w}, y_{w}, z_{w}\right)$, etc. By Proposition A.2, the Hilbert basis is $\mathcal{H}=\left\{p_{1}, \ldots, p_{14}\right\}$, where the polynomial functions $p_{i}: \mathbb{R}^{12} \rightarrow \mathbb{R}, i=1, \ldots, 14$ are defined by

$$
\begin{aligned}
& p_{1}(v, w, u, t)=\|v\|^{2}=x_{v}^{2}+y_{v}^{2}+z_{v}^{2}, \quad p_{2}(v, w, u, t)=x_{w}^{2}+y_{w}^{2}+z_{w}^{2}, \quad p_{3}(v, w, u, t)=x_{u}^{2}+y_{u}^{2}+z_{u}^{2}, \\
& p_{4}(v, w, u, t)=x_{t}^{2}+y_{t}^{2}+z_{t}^{2}, \quad p_{5}(v, w, u, t)=\langle v, w\rangle=x_{v} x_{w}+y_{v} y_{w}+z_{v} z_{w}, \\
& p_{6}(v, w, u, t)=x_{v} x_{u}+y_{v} y_{u}+z_{v} z_{u}, \quad p_{7}(v, w, u, t)=x_{v} x_{t}+y_{v} y_{t}+z_{v} z_{t}, \\
& p_{8}(v, w, u, t)=x_{w} x_{u}+y_{w} y_{u}+z_{w} z_{u}, \quad p_{9}(v, w, u, t)=x_{w} x_{t}+y_{w} y_{t}+z_{w} z_{t}, \\
& p_{10}(v, w, u, t)=x_{u} x_{t}+y_{u} y_{t}+z_{u} z_{t}, \\
& p_{11}(v, w, u, t)=\operatorname{det}(v, w, u)=x_{v} y_{w} z_{u}+x_{w} y_{u} z_{v}+x_{u} y_{v} z_{w}-z_{v} y_{w} x_{u}-y_{v} x_{w} z_{u}-x_{v} z_{w} y_{u}, \\
& p_{12}(v, w, u, t)=\operatorname{det}(v, w, t)=x_{v} y_{w} z_{t}+x_{w} y_{t} z_{v}+x_{t} y_{v} z_{w}-z_{v} y_{w} x_{t}-y_{v} x_{w} z_{t}-x_{v} z_{w} y_{t}, \\
& p_{13}(v, w, u, t)=\operatorname{det}(v, u, t)=x_{v} y_{u} z_{t}+x_{u} y_{t} z_{v}+x_{t} y_{v} z_{u}-z_{v} y_{u} x_{t}-y_{v} x_{u} z_{t}-x_{v} z_{u} y_{t}, \\
& p_{14}(v, w, u, t)=\operatorname{det}(w, u, t)=x_{w} y_{u} z_{t}+x_{u} y_{t} z_{w}+x_{t} y_{w} z_{u}-z_{w} y_{u} x_{t}-y_{w} x_{u} z_{t}-x_{w} z_{u} y_{t} .
\end{aligned}
$$

Since the lengths of the four vectors and their relative positions in space are completely determined by these 14 polynomials, we find, as expected, $\left(p_{1}, \ldots, p_{14}\right)\left(\mathbb{R}^{12}\right) \simeq \mathbb{R}^{12} / \mathrm{SO}(3)$. We compute the vector fields $X_{1}, \ldots, X_{14}$ associated to these polynomials. Since

$$
\begin{aligned}
& \tilde{p}_{1}(v, w)=0, \quad \tilde{p}_{2}(v, w)=0, \quad \tilde{p}_{3}(v, w)=0, \quad \tilde{p}_{4}(v, w)=0, \\
& \tilde{p}_{5}(v, w)=0, \quad \tilde{p}_{6}(v, w)=x_{v} \mathrm{~d} x_{u}+y_{v} \mathrm{~d} y_{u}+z_{v} \mathrm{~d} z_{u}, \quad \tilde{p}_{7}(v, w)=x_{v} \mathrm{~d} x_{t}+y_{v} \mathrm{~d} y_{t}+z_{v} \mathrm{~d} z_{t}, \\
& \tilde{p}_{8}(v, w)=x_{w} \mathrm{~d} x_{u}+y_{w} \mathrm{~d} y_{u}+z_{w} \mathrm{~d} z_{u}, \quad \tilde{p}_{9}(v, w)=x_{w} \mathrm{~d} x_{t}+y_{w} \mathrm{~d} y_{t}+z_{w} \mathrm{~d} z_{t}, \quad \tilde{p}_{10}(v, w)=0, \\
& \tilde{p}_{11}(v, w)=\left(x_{v} y_{w}-y_{v} x_{w}\right) \mathrm{d} z_{u}+\left(x_{w} z_{v}-x_{v} z_{w}\right) \mathrm{d} y_{u}+\left(y_{v} z_{w}-z_{v} y_{w}\right) \mathrm{d} x_{u}, \\
& v_{12}(v, w)=\left(x_{v} Y_{w}-y_{v} x_{w}\right) \mathrm{d} z_{t}+\left(x_{w} z_{v}-x_{v} z_{w}\right) \mathrm{d} y_{t}+\left(y_{v} z_{w}-z_{v} y_{w}\right) \mathrm{d} x_{t}, \\
& \tilde{p}_{13}(v, w)=0, \quad \tilde{p}_{14}(v, w)=0,
\end{aligned}
$$


we find

$$
\begin{aligned}
& X_{1}=X_{2}=X_{3}=X_{4}=X_{5}=X_{10}=X_{13}=X_{14}=0, \\
& X_{6}(v, w)=X_{v} \partial_{X_{v}}+y_{v} \partial_{Y_{v}}+z_{v} \partial_{z_{v}}, \quad X_{7}(v, w)=X_{v} \partial_{X_{w}}+y_{v} \partial_{Y_{w}}+z_{v} \partial_{Z_{w}}, \\
& X_{8}(v, w)=X_{w} \partial_{X_{v}}+y_{w} \partial_{Y_{v}}+z_{w} \partial_{Z_{v}}, \quad X_{9}(v, w)=X_{w} \partial_{X_{w}}+y_{w} \partial_{Y_{w}}+z_{w} \partial_{Z_{w}}, \\
& X_{11}(v, w)=\left(X_{v} Y_{w}-y_{v} X_{w}\right) \partial_{z_{v}}+\left(X_{w} z_{v}-X_{v} z_{w}\right) \partial_{Y_{v}}+\left(y_{v} z_{w}-z_{v} y_{w}\right) \partial_{X_{v}}, \\
& X_{12}(v, w)=\left(X_{v} Y_{w}-y_{v} X_{w}\right) \partial_{z_{w}}+\left(X_{w} z_{v}-X_{v} z_{w}\right) \partial_{Y_{w}}+\left(y_{v} z_{w}-z_{v} y_{w}\right) \partial_{X_{w}} .
\end{aligned}
$$

Thus, $\mathcal{T}_{G}$ is spanned by

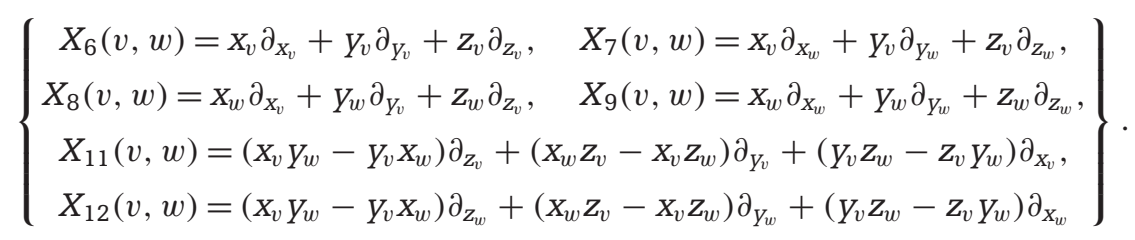

The vector field $Y$ defined by

$$
\begin{aligned}
Y(v, w)= & ((v \times w) \times v)_{X} \partial_{X_{1}}+((v \times w) \times v)_{Y} \partial_{Y_{1}}+((v \times w) \times v)_{z} \partial_{Z_{1}} \\
& +((v \times w) \times w)_{X} \partial_{X_{2}}+((v \times w) \times w)_{Y_{Y_{2}}} \partial_{Y_{2}}+((v \times w) \times w)_{Z} \partial_{Z_{2}} \\
= & \langle v, v\rangle\left(X_{2} \partial_{X_{1}}+y_{2} \partial_{Y_{1}}+z_{2} \partial_{Z_{1}}\right)-\langle v, w\rangle\left(X_{1} \partial_{X_{1}}+y_{1} \partial_{Y_{1}}+z_{1} \partial_{Z_{1}}\right) \\
& +\langle v, w\rangle\left(X_{2} \partial_{X_{2}}+y_{2} \partial_{Y_{2}}+z_{2} \partial_{Z_{2}}\right)-\langle w, w\rangle\left(X_{1} \partial_{X_{2}}+y_{1} \partial_{Y_{2}}+z_{1} \partial_{Z_{2}}\right) \\
= & \langle v, v\rangle X_{8}(v, w)-\langle v, w\rangle X_{6}(v, w)+\langle v, w\rangle X_{9}(v, w)-\langle w, w\rangle X_{7}(v, w)
\end{aligned}
$$

for all $(v, w) \in \mathbb{R}^{3} \times \mathbb{R}^{3}$ is then also an element of $\mathcal{T}_{G}$, where $((v \times w) \times v)_{X},((v \times w) \times v)_{Y}$ and $((v \times w) \times v)_{z}$ are the $x^{-}, Y^{-}$and $z$-components of the vector product $(v \times w) \times v$.

\section{References}

[1] Bierstone, E. "Lifting isotopies from orbit spaces." Topology 14, no. 3 (1975): 245-52.

[2] Blankenstein, G. "Implicit Hamiltonian systems: symmetry and interconnection." $\mathrm{PhD}$ thesis, University of Twente, 2000.

[3] Blankenstein, G. and T. S. Ratiu. "Singular reduction of implicit Hamiltonian systems." Reports on Mathematical Physics 53, no. 2 (2004): 211-60. 
[4] Blankenstein, G. and A. J. van der Schaft. "Symmetry and reduction in implicit generalized Hamiltonian systems." Reports on Mathematical Physics 47, no. 1 (2001): 57-100.

[5] Bourbaki, N. Éléments de mathématique. Fasc. XXXIII. Variétés différentielles et analytiques. Fascicule de résultats (Paragraphes 1 à 7), Actualités Scientifiques et Industrielles 1333. Paris: Hermann, 1967.

[6] Bursztyn, H., G. R. Cavalcanti, and M. Gualtieri. "Reduction of Courant algebroids and generalized complex structures." Advances in Mathematics 211, no. 2 (2007): 726-65.

[7] Chossat, P. and R. Lauterbach. Methods in Equivariant Bifurcations and Dynamical Systems. Advanced Series in Nonlinear Dynamics 15. River Edge, NJ: World Scientific, 2000.

[8] Courant, T. J. "Dirac manifolds." Transactions of the American Mathematical Society 319, no. 2 (1990): 631-61.

[9] Courant, T. J. and A. Weinstein. Beyond Poisson structures, 39-49. Action hamiltoniennes de groupes. Troisième théorème de Lie (Lyon, 1986), Travaux en Cours 27. Paris: Hermann, 1988.

[10] Cushman, R. and J. Śniatycki. "Differential structure of orbit spaces." Canadian Journal of Mathematics 53, no. 4 (2001): 715-55.

[11] Duistermaat, J. J. and J. A. C. Kolk. Lie Groups, Universitext. Berlin: Springer. viii, 344pp., 2000.

[12] Fernandes, R. L., J.-P. Ortega, and T. S. Ratiu. "The momentum map in Poisson geometry." American Journal of Mathematics 131, no. 5 (2009): 1261-310.

[13] Jotz, M. "Dirac Group(oid)s and Their Homogeneous Spaces." PhD thesis, EPFL, Lausanne, 2011.

[14] Jotz, M. and C. Ortiz. "Foliated groupoids and their infinitesimal data." (2011): Preprint, arXiv:1109.4515v1.

[15] Jotz, M. and T. S. Ratiu. "Poisson reduction by distributions." Letters in Mathematical Physics 87, no. 1-2 (2009): 139-47.

[16] Jotz, M. and T. S. Ratiu. "Dirac structures, nonholonomic systems and reduction." Reports on Mathematical Physics (2011): preprint arXiv:0806.1261.

[17] Jotz, M. and T. S. Ratiu. "Induced Dirac structure on isotropy type manifolds." Transformation Groups 16, no. 1 (2011): 175-91.

[18] Jotz, M., T. S. Ratiu, and J. Śniatycki. "Singular Dirac reduction." Transactions of the American Mathematical Society 363, no. 6 (2011): 2967-3013.

[19] Jotz, M., T. Ratiu, and M. Zambon. "Invariant frames for vector bundles and applications." Geometriae Dedicata (2011): 1-12. doi: 10.1007/s10711-011-9618-8.

[20] Kraft, H. and C. Procesi. "Classical Invariant Theory, A Primer." Available at www.math. unibas.ch/ kraft/Papers/KP-Primer.pdf (1996).

[21] Libermann, P. and C.-M. Marle. Symplectic Geometry and Analytical Mechanics. Transl. from the French by Bertram Eugene Schwarzbach., Mathematics and its Applications 35. Dordrecht: D. Reidel Publishing Company, a member of the Kluwer Academic Publishers Group. XVI, 526 p., 1987. 
[22] Marsden, J. E. and T. S. Ratiu. "Reduction of Poisson manifolds." Letters in Mathematical Physics 11, no. 2 (1986): 161-9.

[23] Ortega, J.-P. and T. S. Ratiu. Momentum Maps and Hamiltonian Reduction. Progress in Mathematics 222. Boston, MA: Birkhäuser. xxxiv, 497pp., 2004.

[24] Pflaum, M. J. Analytic and Geometric Study of Stratified Spaces, Lecture Notes in Mathematics 1768. Berlin: Springer, 2001.

[25] Śniatycki, J. "Orbits of families of vector fields on subcartesian spaces." Université de Grenoble. Annales de l'Institut Fourier 53, no. 7 (2003): 2257-96.

[26] Stefan, P. "Accessibility and foliations with singularities." Bulletin of the American Mathematical Society 80, no. 6 (1974): 1142-45.

[27] Stefan, P. "Accessible sets, orbits, and foliations with singularities." Proceedings of the London Mathematical Society (3) 29, no. 4 (1974): 699-713.

[28] Stefan, P. "Integrability of systems of vector fields." Journal of London Mathematical Society (2) 21, no. 3 (1980): 544-56.

[29] Sussmann, H. J. "Orbits of families of vector fields and integrability of distributions." Transactions of the American Mathematical Society 180 (1973): 171-88.

[30] Vaisman, I. Lectures on the Geometry of Poisson Manifolds. Progress in Mathematics 118. Basel: Birkhäuser, 1994.

[31] Yoshimura, H. and J. E. Marsden. "Reduction of Dirac structures and the HamiltonPontryagin principle." Reports on Mathematical Physics 60, no. 3 (2007): 381-426.

[32] Yoshimura, H. and J. E. Marsden. “Dirac cotangent bundle reduction." Journal of Geometric Mechanics 1, no. 1 (2009): 87-158. 